\title{
Mapping Water Ice Clouds on Mars with MRO/MARCI
}

\author{
Michael J. Wolff ${ }^{\mathrm{a}}$, R. Todd Clancy ${ }^{\mathrm{a}}$, Melinda A. Kahre ${ }^{\mathrm{b}}$, Robert M. \\ Haberle $^{\mathrm{b}}$, François Forget ${ }^{\mathrm{c}}$, Bruce A. Cantor ${ }^{\mathrm{d}}$, Michael C. Malin ${ }^{\mathrm{d}}$ \\ ${ }^{a}$ Space Science Institute, 4750 Walnut Street, Suite 205, Boulder, CO 80301, UCB 564 \\ ${ }^{b}$ NASA Ames Research Center, Moffat Field, CA 94035, USA \\ ${ }^{c}$ Laboratoire de Météorologie Dynamique/IPSL, Sorbonne Université, École Normale \\ supérieure, PSL Research University, École polytechnique, CNRS, Paris, France \\ ${ }^{d}$ Malin Space Science Systems, P.O. Box 910149, San Diego, CA 92191, USA
}

\section{Abstract}

Observations by the Mars Color Imager (MARCI) onboard the Mars Reconnaissance Orbiter (MRO) in the ultraviolet (UV, Band 7; $320 \mathrm{~nm}$ ) are used to characterize the spatial and temporal behavior of atmospheric water ice over a period of 6 Mars Years. Exploiting the contrast of the bright ice clouds to the low albedo surface, a radiative transfer-based retrieval algorithm is developed to derive the column-integrated optical depth of the ice $\left(\tau_{\text {ice }}\right)$. Several relatively unique input products are created as part of the retrieval development process, including a zonal dust climatology based on emission phase function (EPFs) sequences from the Compact Reconnaissance Imaging Spectrometer for Mars (CRISM), a spatially variable UV-reflectance model for Band 7 (as well as for Band 6, $260 \mathrm{~nm}$ ), and a water ice scattering phase function based on a droxtal ice habit. Taking into account a radiometric precision of $7 \%$, an error analysis estimates the uncertainty in $\tau_{\text {ice }}$ to be $\sim 0.03$ (exluding particle size effects, which are discussed separately).

URL: mjwolff@spacescience.org, 262-790-1356 (Michael J. Wolff) 
Zonal trends are analyzed over the full temporal extent of the observations, looking at both diurnal and interannual variability. The main (zonal) features are the aphelion cloud belt $(\mathrm{ACB})$ and the polar hoods. For the ACB, there can be an appreciable diurnal change in $\tau_{i c e}$ between the periods of 14h30-15h00 and 15h00-15h30 Local True Solar Time (LTST). The amplitude of this effect shows relatively large interannual variability, associated mainly with changes in the earlier time block. When averaged over the interval 14h00-16h00 LTST, the interannual differences in the ACB structrure are appreciably smaller. When the MARCI $\tau_{i c e}$ are compared to those from the Thermal Emission Spectrometer (TES), there is a good correlation of features, with the most significant difference being the seasonal $\left(\mathrm{L}_{S}\right)$ evolution of the ACB. For TES, the ACB zonal profile is relative symmetric about $\mathrm{L}_{S}=90^{\circ}$. In the MARCI data, this profile is noticeably asymmetric, with the centroid shifted to later in the northern summer season $\left(\mathrm{L}_{S}=120^{\circ}\right)$. The MARCI behavior is consistent with that observed by several other instruments. The correspondence of MARCI $\tau_{i c e}$ zonal and meridional behavior with that predicted by two Global Circulation Models (GCM) is good. Each model captures the general behavior seen by MARCI in the ACB, the polar hoods, and the major orographic/topographic cloud features (including Valles Mariners). However, the mismatches between GCM results and MARCI reinforce the challenging nature of water ice clouds for dynamical models. The released $\tau_{i c e}$ are being archived at Malin Space Science Systems at https://www.msss.com/mro_marci_iceclouds/.

Keywords: MARS, ATMOSPHERE, ULTRAVIOLET OBSERVATIONS, RADIATIVE TRANSFER 


\section{Introduction}

The presence of ice clouds in the Martian atmosphere was originally inferred from their color contrast with the "yellow hazes" observed in early telescopic photographs (e.g. Slipher, 1962). The specific identification of water ice resulted from analysis of Mariner 9 Infrared Interferometer Spectrometer observations of clouds over the Tharsis ridge (Curran et al., 1973). The imaging observations compiled by the Mariner 9 and the Viking orbiters characterized the general morphology and large-scale (qualitative) behavior of Martian water ice clouds (e.g. Leovy et al., 1973; Anderson and Leovy, 1978; Conrath et al., 1973; French et al., 1981; Kahn, 1984). While these efforts offered insight into the physical forms taken by clouds and their association with meterological (and dynamical) phenomena, clouds were viewed primarily as passive tracers or probes of more fundamental atmospheric behavior. This picture began to change in the mid-1990's with the realization that water ice clouds could be active participants in fundamental transport, radiative, and photochemical processes (Clancy et al., 1996; Clancy and Nair, 1996). This paradigm shift, combined with the resumption of Mars spacecraft missions, stimulated additional theoretical efforts to better understand the impact of clouds on the Martian atmosphere and climate (e.g. Richardson et al., 2002; Montmessin et al., 2004; Lefèvre et al., 2008; Wilson et al., 2008; Madeleine et al., 2012a; Navarro et al., 2014).

The aforementioned renaissance of Martian spacecraft observations began with the orbital insertion of Mars Global Surveyor in 1997 (MGS; Albee et al., 1998). MGS was subsequent joined by Mars Odyssey (Garvin et al., 2001), Mars Express (Schmidt, 2003), and Mars Reconnaissance Orbiter (MRO; 
Zurek and Smrekar, 2007). For a review of the specific investigations into water ice clouds, we refer the interested reader to the reviews by Smith (2008) and by Clancy et al. (2017). For our purposes, we wish simply to highlight three general areas of water ice cloud properties that have been enabled by the post-Viking data sets. Specifically, one finds the ability to synoptically measure the water ice column, including seasonal and interannual variability (Pearl et al., 2001; Clancy et al., 2003; Wolff and Clancy, 2003; Smith, 2004; Mateshvili et al., 2007; Madeleine et al., 2012b), to retrieve basic microphysical properties such as particle size (Clancy et al., 2003; Wolff and Clancy, 2003; Zasova et al., 2005; Madeleine et al., 2012b; Olsen et al., 2019), and to characterize the vertical distribution of water ice (Benson et al., 2010, 2011; Smith et al., 2013). Much of the work in these topics has been driven by the data produced by spectrometers and radiometers: i.e., Thermal Emission Spectrometer (TES; Christensen et al., 2001), Thermal Emission Imaging System (THEMIS; Christensen et al., 2004). Spectroscopy for the Investigation of the Characteristics of the Atmosphere of Mars (SPICAM; Bertaux et al., 2006), Observatoire pour la Minéralogie, l'Eau, les Glaces et l'Activité (OMEGA; Bibring et al., 2004), Planetary Fourier Spectrometer (PFS; Formisano et al., 2005), Mars Climate Sounder (MCS; McCleese et al., 2007)), and Compact Reconnaissance Imaging Spectrometer for Mars (CRISM; Murchie et al., 2007). The concentration of effort on these datasets is due, at least in part, to the diagnostic power of the unique geometrical and wavelength coverage offered by the aforementioned instruments. However, the same attributes that allow for the utility of the data can also contribute to inherent limitations in the resulting datasets with respect to spatial and 
temporal sampling restrictions. However, complementary datasets exist in the form of images that cover large portions of the planet with systematic temporal cadences. More specifically, we refer to the data obtained by widefield camera systems.

No past or current spacecraft mission to Mars has flown without some type of imaging system (though in a very recent case this is an imaging spectrometer system). Nevertheless, the Mars Observer Camera (MOC; Malin et al., 1992) onboard MGS is the first example of an imager that routinely covers (essentially) the entire illuminated portion of the planet over a very short timescale (i.e., a day). This capability led to some qualitative global-scale and quantitative regional-scale characterizations of water ice cloud columns that were not previously possible (e.g., Wang and Ingersoll, 2002; Benson et al., 2003, 2006; Wang and Fisher, 2009). The small number of studies that have exploited this extensive dataset (daily global coverage from 1999 through 2006) is partially a function of its large size, but also the lack of a relatively accurate photometric calibration until relatively late in the MGS mission (James et al., 2007). In addition, even with the use of the Wide Angle blue channel to maximize the discrimination between dust and ice, the variability of surface photometric properties represents a significant complication for quantitative analysis. Ultimately, the arrival of MRO and its Mars Color Imager (MARCI; Malin et al., 2008) created the opportunity to combine global-scale imaging with specific sensitivity to water ice aerosols.

From the perspective of water ice cloud studies, the MARCI dataset possesses two distinct advantages over that compiled by MOC. Namely, the MARCI instrument is designed to have higher photometric fidelity and to 
include a wavelength region which maximizes the atmospheric radiometric signature produced by water ice clouds with respect to the the surface contribution. As a result, MARCI data are more amenable to the robust retrieval of (column integrated) water ice optical depths. Although the sun-synchronous aspect of the MRO orbit limits the local time coverage available, particularly in the mid- and the low- latitude regions, it is the goal of this paper to describe an algorithm that produces a "daily global map" (DGM) of water ice optical depth $\left(\tau_{i c e}\right)$ and demonstrates its utility for Martian atmospheric studies, including applications such as Global Climate Models (GCMs).

We begin with a brief overview of the MARCI observations that form the basis of the water ice optical retrievals. Because the MARCI instrument has been described elsewhere, we limit ourselves to the details necessary to provide context for subsequent sections. An exception to this is the updated calibration and model input parameters (e.g., camera model, radiative properties). Since the derivation of such values was generally done for both UV channels at the same time, it is natural to include both sets of results here. This also supports their use in the ozone retrievals of Clancy et al. (2016). In addition, given the utility of near-contemporaneous dust optical measurements in our work, we include a brief description of the dust dataset employed. Section 3 provides the details of the MARCI retrieval scheme, including a delineation of the major input parameters and associated constraints. An example of the resulting DGMs for $\tau_{i c e}$ is presented in Section 4, along with a discussion of the various sources of uncertainty and error. Section 5 includes an examination of the MARCI water ice cloud retrievals in terms of temporal and spatial behavior, particularly with respect 
to previous observations and to the predictions by Mars Global Circulation Models (MGCMs). Finally, we briefly discuss the status of the distribution of available MARCI $\tau_{\text {ice }}$ products.

\section{Observations}

Our retrieval of water ice cloud optical depths focuses on the images produced by the longer wavelength channel of the two MARCI UV bands, a.k.a., Band $7(\lambda \sim 320 \mathrm{~nm})$. However, given the ubiquitous nature of atmospheric dust and the sensitivity of the shorter wavelength channel (Band $6, \lambda \sim 260 \mathrm{~nm})$ to molecular absorption by ozone, the UV data are not sufficient to unambiguously derive the atmospheric columns of both dust and ice aerosols. The MARCI visible images only become useful for this purpose under very dusty conditions, and even then only marginally so. Consequently, we also use the dust optical depths derived from the Compact Reconnaissance Imaging Spectrometer (CRISM; Murchie et al., 2007).

\subsection{MARCI Data}

The MARCI instrument is a wide-angle, 7-band "push-frame" imaging sytem whose components, capabilities, and performance are described in significant depth in three overview papers (Malin et al., 2001; Malin et al., 2008; Bell et al., 2009). Additional aspects specific to the UV images may be found in Wolff et al. (2010, hereafter W10) and Clancy et al. (2016). In the interest of brevity, we recount only the details needed for the presentation of the water ice optical depths. However, in support of the previously reported ozone results (Clancy et al., 2016), we do include such summary information for both UV channels. 


\subsubsection{UV Image and Radiometric Characteristics}

The spatial sampling provided by the UV images is set by the $8 \mathrm{x} 8$ summing that is done by the instrument, whose primary goal is to increase the signal-to-noise ratio in Band 6 (e.g., Bell et al., 2009). This provides a downlinked pixel field-of-view of approximately $8 \mathrm{~km}$ at nadir, compared to the nominal $\sim 1 \mathrm{~km}$ per pixel value for the visible bands. As discussed by Clancy et al. (2016), the daily "global coverage" is created from the 13-14 south-tonorth mapping swaths obtained (each Mars day) from the sun-synchronous orbit of MRO. The center line of these swaths is separated by about $27^{\circ}$ in longitude and has a local time near $15 \mathrm{~h} 00$ local true solar time (LTST) (and 03h00 LTST on the nightside). Due to calibration and performance limitations, we generally discard the data for emergence angles greater than $70^{\circ}$. Figure 1 illustrates the resulting spatial coverage for a typical day in the northern and southern mid-summer seasons. For context, the quantity plotted is the normalized radiance, known as the radiance factor or I/F (see $\S 3.1$. The brightening of the image strip near the edge of the images is primarily associated with the increasing effect of the aerosols as the air mass (emergence angles) increase both sides of the nadir point; however diurnal variations can be present as on well.

The solar irradiance-weighted centroid values for the system response functions of Band 6 and 7 are $263 \mathrm{~nm}$ and $321 \mathrm{~nm}$, respectively, with the fullwidth at half-max (FWHM) values of $30 \mathrm{~nm}$ and $24 \mathrm{~nm}$. A combination of ground and in-flight calibration efforts has derived radiometric coefficients of $1.15 \times 10^{-2}$ and $2.50 \times 10^{-2}$ (DataNumber/millisecond) per $\left(W / m^{2} / \mu \mathrm{m} / \mathrm{sr}\right)$

for Band 6 and 7, with associated absolute uncertainties estimated as $\lesssim 10 \%$ 

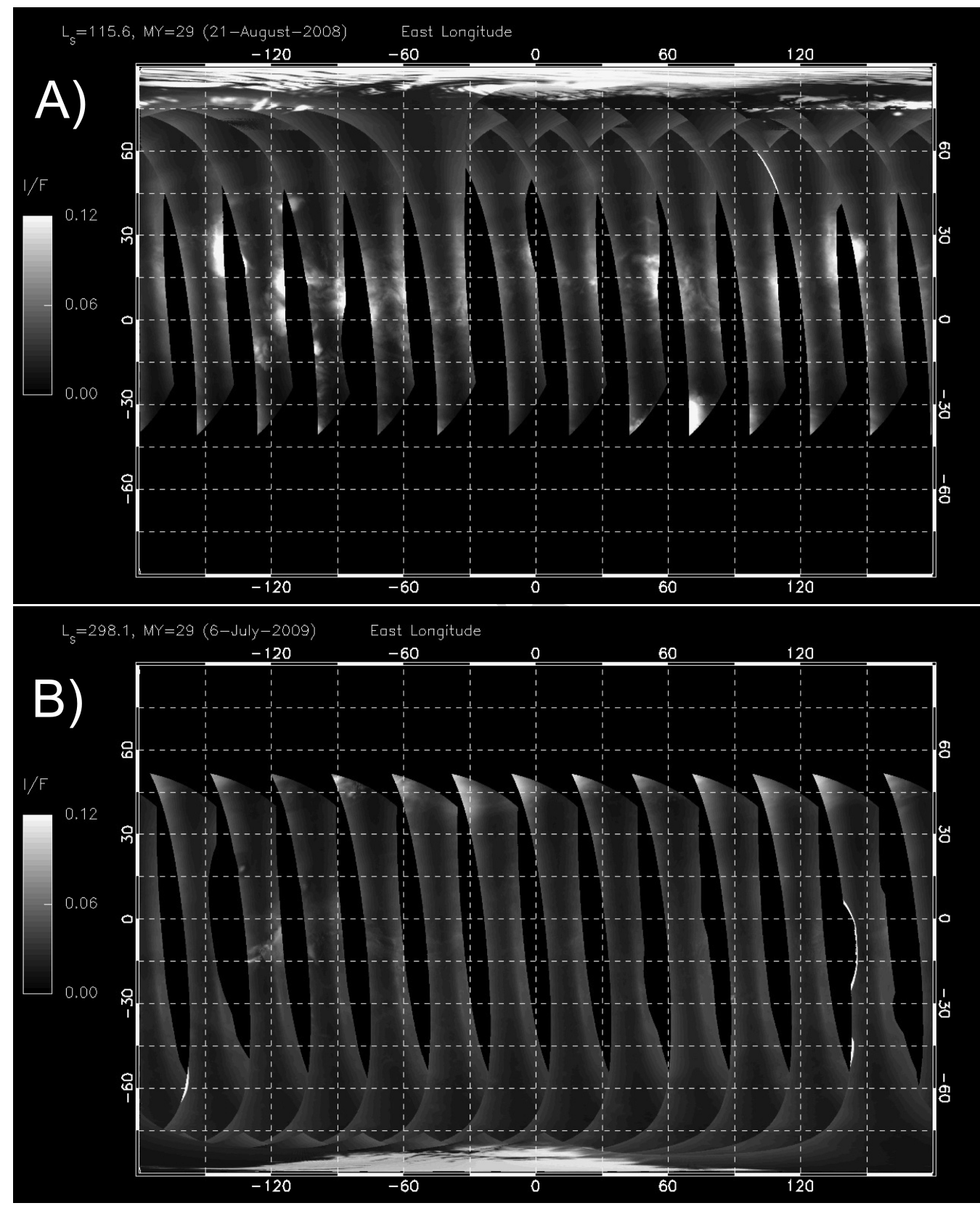

Figure 1: UV spatial coverage for a given mapping day in (A) northern mid-summer $\mathrm{L}_{S}=116^{\circ}$, and (B) southern mid-summer $-\mathrm{L}_{S}=298^{\circ}$. The cylindrical projection of the Band 7 data emphasizes both the uniform, but non-overlapping sampling at middle and low latitudes. Although more difficult to see in this representation, there is significant overlap between swathes at high latitudes, ayowing for a greater sampling of local time in the polar regions. Pixels with emergence and incidence angles greater than $70^{\circ}$ and $75^{\circ}$, respectively, are excluded from each projection. 
$\sim 5-8 \%$, respectively. The Band 6 value represents a $6 \%$ adjustment by Clancy et al. (2016) from that of W10. The relative precision (across the field of view) for both channels is of the order 2-3\% (i.e., W10).

\subsubsection{Camera Model Update}

The pre-flight details of the MARCI geometrical calibration and camera model have been presented in a technical report by Malin Space Science Systems (Malin Space Science Systems (MSSS), 2005). Semenov (2007) offers a subsequent update using on-orbit observations, and also provides a practical description of the model implementation within the framework of Planetary Data System Navigation Node software (http://naif.jpl.nasa.gov/naif/). More specifically, Semenov describes the basic algorithms necessary to calculate the distorted and undistorted pixel geometries using the relevant quantities from the MARCI Instrument Kernel file (IK, mro_marci_v10.ti). However, the updated camera parameters are based on data from the visible camera. As can be seen in the top panel of Figure 2, the UV camera values are in need of some additional refinement.

To better characterize the camera errors as a function of viewing geometry, we used a set of observations from northern summer where there is an appreciable number of features available for registration. Selecting a sample of data from September 2014, we generated a series of stereographic (north) polar projections using a grid of $\mathrm{x}$ - and $\mathrm{y}$-offsets from the previously reported position of the optical axis were generated, where the goal was to minimize the errors associated with simple shifts. Contemporaneous Band 3 observations were used as the reference or truth-set. A second step involved the parameterization of the distortion in the discrete structure of the polar cap 

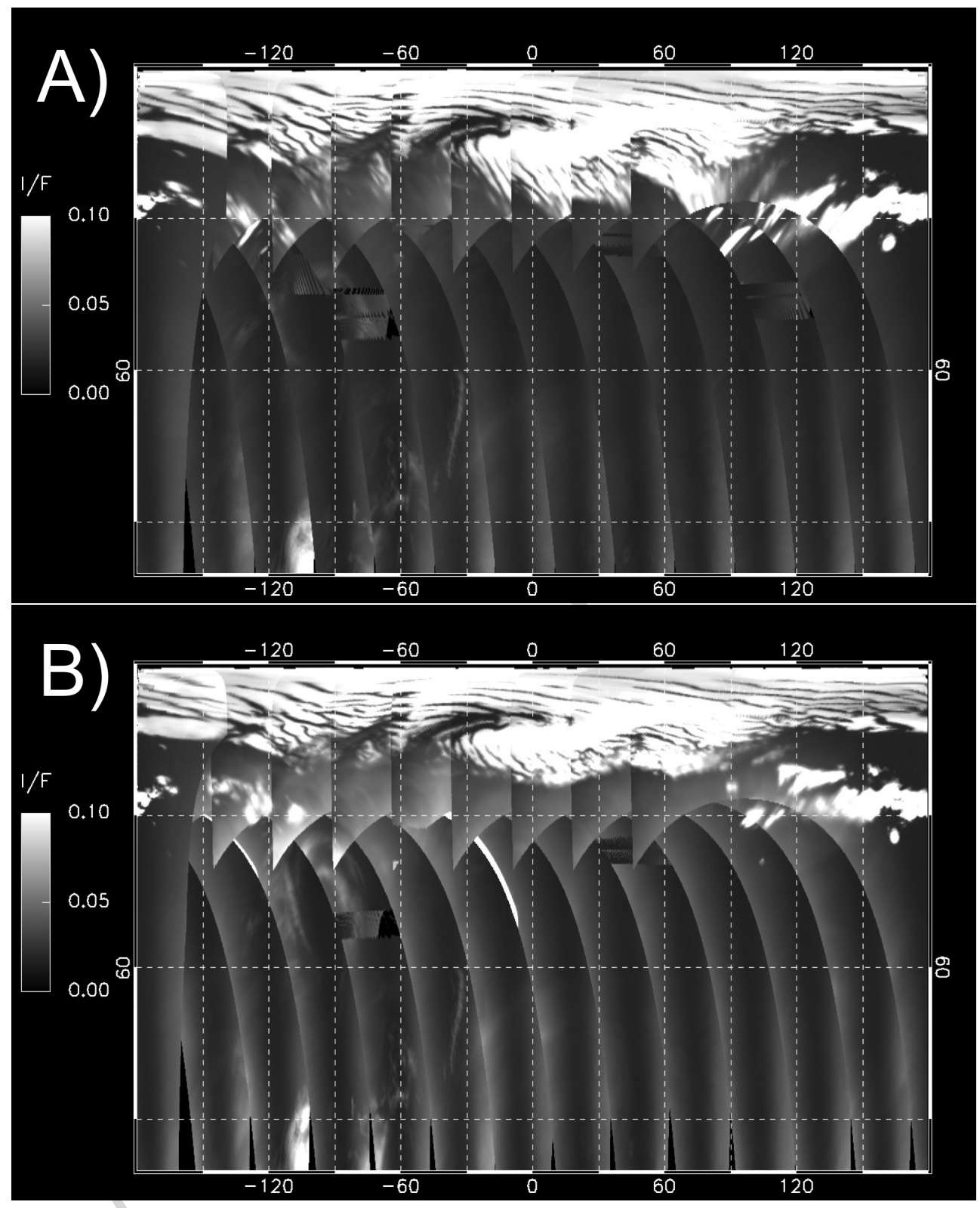

Figure 2: Cylindrical map projection of Band 7 data from 14-September-2008 $\left(\mathrm{L}_{S}=126.8^{\circ}\right)$, cropped to emphasize the high northern latitudes where the overlap between subsequent mapping passes clearly highlights remaining issues with the UV camera model. A) Projection calculated using camera parameters listed in Semenov (2007). B) Projection using updated values (see Table 1). 
in the the outer portions of each mapping swath, which is quite clearly visible in the cylindrical projection in the top panel of Figure 2. The distortion was examined as a function of viewing geometry (e.g., similar to the "mapping angle" discussed in Malin Space Science Systems (MSSS) (2005)), and using Band 3 as a reference. The coefficients of the instrument kernel distortion polynomial (Semenov, 2007)) were adjusted to provide a consistent mapping of surface features with the data sample. The improvements may be seen in the bottom panel of Figure 2, particularly when compared to the top panel. We list the updated camera parameters (as well as the old ones) in Table 1.

\subsection{CRISM Dust Optical Depth Database}

The methodology for the retrieval of the dust column-integrated optical depths is developed by Wolff et al. (2009). While the primary application in that work is the characterization of dust optical properties during the 2007 planet-encircling dust event, a generalization to more diffuse loading cases may also be found there. When this algorithm is applied to the emission phase function (EPF) sequences from early November 2006 through December 2011, one obtains a database containing approximately 24000 optical depth values. This number excludes approximately 2000 EPFs that include observations over ice covered surfaces or for which the model-data fit is unacceptable (typically caused by problems with input data such as extreme photometric angles or truncated observations). While this may appear to be a large number of data points, the spatial-temporal coverage is not adequate to provide a simple spatial interpolation scheme without extremely coarse temporal resolution, i.e., a large fraction of a season. In fact, one must employ a combination of zonal-mean averaging with $10^{\circ}$ latitude bins 
Table 1: updates to the instrument kernel parameters for the marci uv bands

\begin{tabular}{ccccccc}
\hline \multirow{2}{*}{ parameter name } & \multicolumn{2}{c}{ band 6 values $^{\mathrm{a}}$} & \multicolumn{2}{c}{ band 7 value $^{\mathrm{b}}$} & notes $^{\mathrm{c}}$ \\
& old & new & old & new & \\
\hline INS-74400_BAND_CCD_OFFSET & 7 & -1 & -20 & -28 & \\
INS-74400_BAND_CCD_SAMPLE_OFFSET & 0 & 6 & 0 & 6 & $\mathrm{~A}$ \\
$c_{0}$ & 1.02 & 0.95 & 1.01661 & 0.95 & $\mathrm{~B}$ \\
$c_{1}$ & $2.63 \mathrm{e}-06$ & $1.5 \mathrm{e}-6$ & $2.63 \mathrm{e}-06$ & $1.5 \mathrm{e}-6$ & $\mathrm{~B}$ \\
$c_{2}$ & $2.35 \mathrm{e}-12$ & $-8.2 \mathrm{e}-12$ & $2.35 \mathrm{e}-12$ & $-8.2 \mathrm{e}-12$ & $\mathrm{~B}$ \\
$c_{3}$ & $1.25 \mathrm{e}-16$ & $8.0 \mathrm{e}-17$ & $1.25 \mathrm{e}-16$ & $8.0 \mathrm{e}-17$ & $\mathrm{~B}$ \\
$c_{4}$ & 0. & $2.0 \mathrm{e}-23$ & 0. & $2.0 \mathrm{e}-23$ & $\mathrm{~B}, \mathrm{C}$ \\
\hline
\end{tabular}

a band 6 has the NAIF BAND ID of -74421 .

b band 7 has the NAIF BAND ID of -74422 .

c A. This parameter does not exist in the current documentation for the calculation of distorted_view[0] because it is assumed to be zero for all bands. however, because the uv bands require non-zero values, the new formula is DISTORTED_VIEW[0] = BAND_SAMPLE - CENTER_SAMPLE_INDEXES[BAND_NUMBER] - BAND_CCD_SAMPLE_OFFSET[BAND_NUMBER].

B. distortion polynomial $f=c_{0}+c_{1} r_{d}^{2}+c_{2} r_{d}^{4}+c_{3} r_{d}^{6}+c_{4} r_{d}^{8}, r_{d}$ is the distorted distance in the focal plane. C. $c_{4} r_{d}^{8}$ term is not used by Semenov (i.e., $c_{4}=0$ ), but is required for the UV camera model. 
and time-steps of $22.5^{\circ}$ (in $\mathrm{L}_{S}$ ) to generate the zonal dust climatology shown in Figure 3. Here, the upper panel illustrates the basic retrievals binned as described while the lower panel includes the effects of additional filtering (e.g., outliers in a single bin, clipping the polar hood) and of interpolation to fill in the empty bins that are bracketed by "good" data. This database of optical depths is referenced to a wavelength of $900 \mathrm{~nm}$ and normalized to a surface elevation of $0 \mathrm{~m}$, i.e., the aeroid. We calculate this normalization using the Mars Orbiter Laser Altimeter topography (Smith et al., 1999) and the assumption of an exponential atmosphere with a scale-height of $10 \mathrm{~km}$. The uncertainty associated with the dust optical depth for an individual bin is estimated to be $\max \left(10 \% \tau_{d u s t}, 0.1\right)$, which includes both the accuracy of the retrieval process and the scatter associated with multiple retrievals points in the bin.

The values in Figure 3 are the result of an iterative process, where the dust optical depths $\left(\tau_{\text {dust }}\right)$ are derived ultimately by including the water ice optical depth derived from contemporaneous MARCI observations; by default, every CRISM EPF should occur within a MARCI image swath). In other words, the dust column for each CRISM EPF observation is initially computed under the assumption of no water ice. This set of dust columns was used to create the initial version of the dust climatology employed to retrieve water ice optical depths from MARCI images for the period of November 2006 through December 2011. The resulting MARCI $\tau_{i c e}$ values associated with each EPF spatial footprint are then used as an input in the next iteration of the CRISM EPF retrieval. This sequence was repeated three times, at which time there is essentially no change observed in either $\tau_{d u s t}$ or $\tau_{i c e}$ in the third 


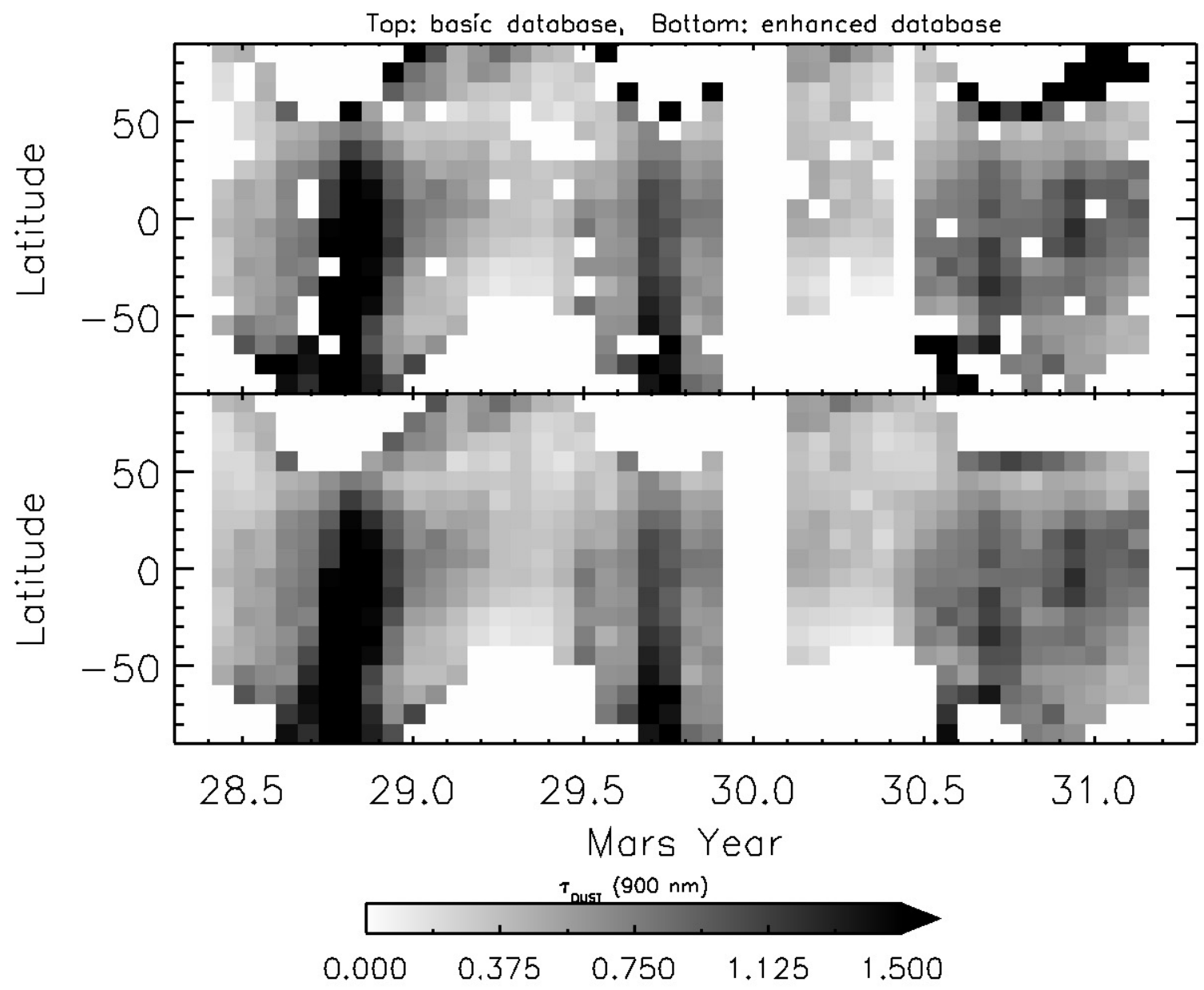

Figure 3: CRISM zonal-mean dust map. The top panel is the result of binning approximately 24000 dust optical depth retrievals using a zonal scheme with widths of $10^{\circ}$ in latitude and $22.5^{\circ}$ in $\mathrm{L}_{S}$. The bottom panel includes the effects of additional filtering for bad retrievals and of interpolation to fill in empty bins. The optical depths are defined at a wavelength of $900 \mathrm{~nm}$ and normalized to an altitude of 0 meters. The gap centered around the start of Mars Year 30 represents the extended spacecraft "safing" event in the latter part of 2009 . 
iteration.

\section{Retrieval Algorithms and Methodology}

The actual transformation of the MARCI Band 7 observations from ra-

diance to $\tau_{i c e}$ is a direct and efficient computational task. By fixing the other input parameters and exploiting the monotonic nature of adding a very bright scatterer above a dark surface, the deriviation of $\tau_{i c e}$ does not need to involve the traditional non-linear approach. Instead, the process is a one-to-one mapping of radiance to $\tau_{i c e}$. Furthermore, the use of a multidimensional lookup table (LUT) and standard interpolation techniques allow one to dispense with computationally inefficient "live" calls to a multiplescattering radiative transfer code. In this section, we provide an overview of the retrieval algorithm from the initial data processing step through the $\tau_{\text {ice }}$ retrieval. This is followed by a description of the LUT and the various input parameters that represent the "physics" of the atmospheric state vector and the boundary conditions.

\subsection{Retrieval Procedure}

The methodology by which the MARCI observations are converted from raw 8-bit data number (DN) values to a water ice optical depth consists of several discrete steps. Although the cadence of MRO orbit and the terrestrial day provides for the natural grouping of the 12-13 swaths of images per day as a "mapping day," we treat a single MARCI image (or swath) as the basic unit for the processing algorithms.

1. Generation of radiometrically calibrated images. The procedure for taking MARCI raw 8-bit DN data (i.e., Level 0 Experiment Data 
Records) to calibrated radiance is well described by Bell et al. (2009). Although we implement these processing steps using an IDL-based set calibration routines, the United States Geological Service's ISIS 3 software replicates this capability with its MARCICAL tool. Here, we include the additional step of removing the dependence of the observed radiance on solar distance by normalizing the data to incident solar radiance. The resulting quantity is the so-called I/F or radiance factor, as defined by Hapke (2012, p. 264). Specifically, I is the observed radiance, and $\mathrm{F}$ is the radiance that would be observed for a perfect Lambert surface illuminated normally by the top-of-the-atmosphere solar flux $(\mathrm{J}): F=J / \pi$. The Mars-Sun distance is calculated for the relevant spacecraft clock time from the MRO and Mars SCLK (spacecraft clock) and PCK (planetary constants) kernels. These data files and core SPICE software libraries are distributed by NASA's Navigation and Ancillary Information Facility (NAIF).

2. Geographic, Photometric, and Reflectance Information. For each pixel in a given image, we calculate the photometric angles (incidence, emergence, and phase) and location information (latitude, longitude). The elevation of the surface is taken explicitly into account for these calculations using the Mars Orbiter Laser Altimeter (MOLA) derived products (i.e., Smith et al., 1999, ; see also http://pds-geosciences. wustl. edu/missions/mgs/mola.html). Finally, a surface reflectance model value (the w parameter decribed later) is assigned to each pixel using the Band 7 map product discussed below in Section 3.4. 
3. Dust column $\left(\tau_{\text {dust }}\right)$ and surface pressure $\left(P_{\text {surf }}\right)$. These two quantities are derived from the CRISM zonal optical depth database discussed in Section 2.2 and from a surface pressure field calculated by the Ames GCM (Smith, 2004). Specifically, we set the $\tau_{\text {dust }}$ and $P_{\text {surf }}$ for the set of nadir pixels in each MARCI image, with the cross-track pixel values populated using the MOLA surface elevations and the assumptions of uniform vertical mixing (with respect to gas density) in an exponential atmosphere with scale height of $10 \mathrm{~km}$ for both gas and dust. The nadir $\tau_{\text {dust }}$ are interpolated in latitude and $\mathrm{L}_{S}$ from the CRISM climatology.

4. At this point, we have specified six of the seven variables in the LUT described in 3.2 and listed in Table 2. It is now a simple process to derive $\tau_{\text {ice }}$ for each pixel through interpolation. A pixel mask is also created to reflect any parameter value (for a given pixel) that is outside of the ranges in the LUT. A common example of this would be for emergence or incidence angles being too large. In addition to diagnostic information, the mask is used to discriminate between $\tau_{i c e}=0$ due to the absence of clouds caused by an input parameter out of range. Figure 4 shows an example of a calibrated I/F image from a northern summer solstice observation and the resulting $\tau_{i c e}$ image.

5. Surface Ice Flag. The retrieval cannot practically distinguish between surface and atmospheric ice. In our implementation, we assume that any radiance needed above the $\tau_{i c e}=0$ case (after all other dimensions are fixed) must be provided by atmospheric water ice above a nonicy surface. As a result, an attempt to flag potential surface ice pixels occurs necessarily in a post-processing step. As can be seen in Figure 4, 


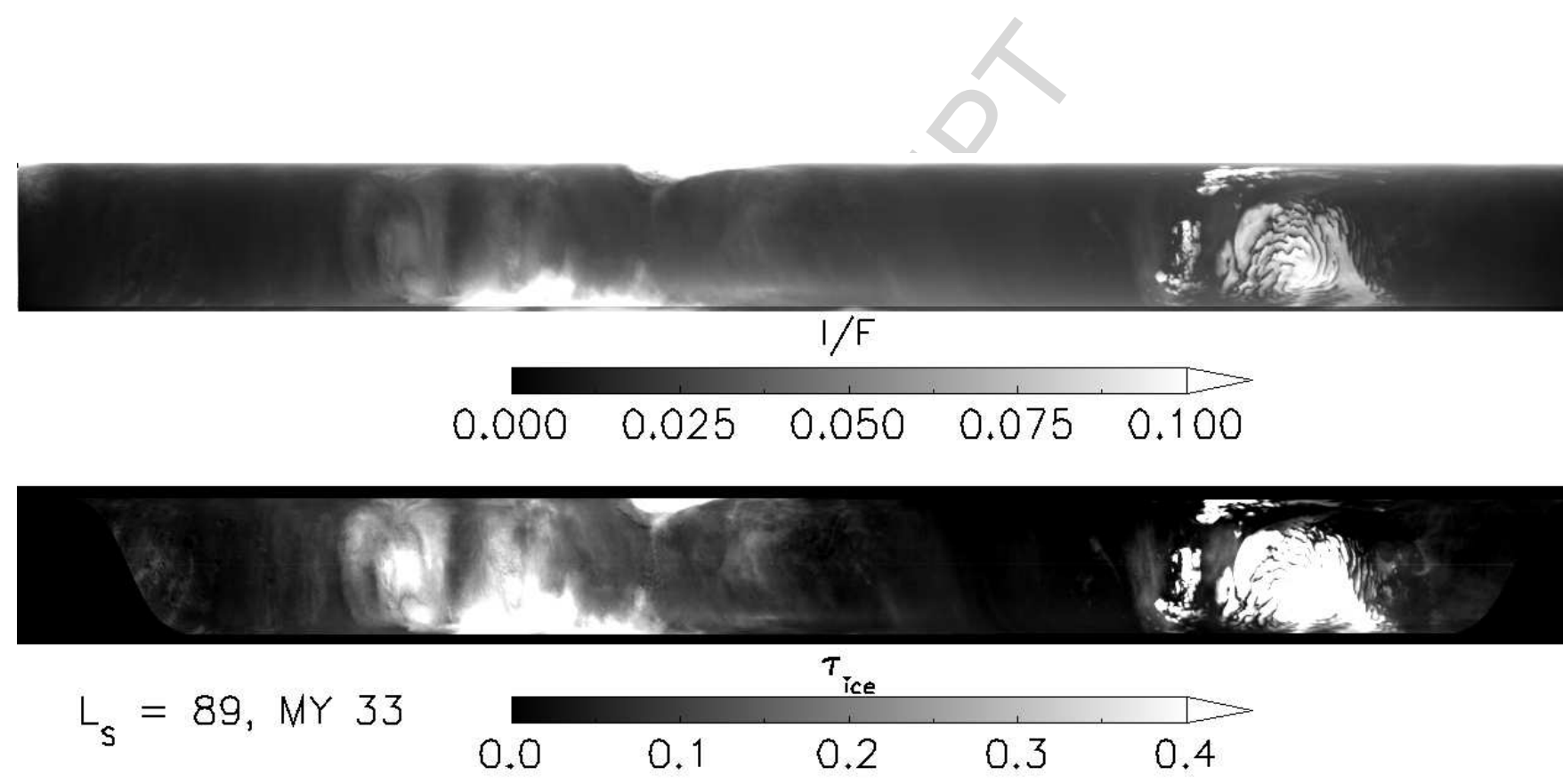

Figure 4: An example I/F image and the resulting $\tau_{i c e}$ product that includes part of the Tharsis ridge near northern solstice in MY 33. The longitude of the nadir point at the equator crossing is $236^{\circ} \mathrm{E}$. The ranges of latitudes starts near $-45^{\circ}$ on the left side of the imaging, passes over the pole and ends near $60^{\circ} \mathrm{N}$. The images are oriented such that North is to the right and West is up. This figure also demonstrates the retrieval limitation associated with surface ice, suggesting the utility of a post-processing step to identify pixels that are likely to include surface ice. See text. 
thick clouds are "derived" for pixels within the north polar residual cap and outliers. However, one might also notice that in addition to the proximity to the north pole, these pixels are quite bright in I/F compared to those of the more diffuse cloud features. We combine these these aspects, location and brightness, into a set of criteria for flagging potential surface ice.

In terms of location, we use the polar cap regression analysis of Cantor et al. (2010) for the north polar region, as well as a similar unpublished analysis for the south cap. We flag a pixel as potentially including surface ice if it is poleward of the notional cap edges as defined below:

$$
\begin{gathered}
\text { lat }_{N}=59^{\circ}+0.214 \mathrm{~L}_{S}-C, \quad 0^{\circ} \leq \mathrm{L}_{S} \leq 90^{\circ} \\
\text { lat }_{N}=59^{\circ}+0.214\left(180^{\circ}-\mathrm{L}_{S}\right)-C, \quad 90^{\circ} \leq \mathrm{L}_{S} \leq 180^{\circ} \\
\operatorname{lat}_{N}=59^{\circ}, \text { otherwise. } \\
\text { lat }_{S}=-12^{\circ}-0.247 \mathrm{~L}_{S}, \quad 180^{\circ} \leq \mathrm{L}_{S} \leq 270^{\circ} \\
\text { lat }_{S}=-12^{\circ}-0.247\left(540^{\circ}-\mathrm{L}_{S}\right), \quad 270^{\circ} \leq \mathrm{L}_{S} \leq 360^{\circ} \\
\operatorname{lat}_{S}=-57^{\circ}, \text { otherwise, }
\end{gathered}
$$

where lat ${ }_{S}$ and lat $N$ are the latitudes of the southern and northern cap edges, respectively. The offset $C$ compensates for the fact that the original regression formulae were designed to track the seasonal cap. However, not all of the outliers in the north polar region are inside the boundaries defined. For example, the southern most outliers in the Figure 4 are $\sim 2-3^{\circ}$ southward of the notional boundary of $78^{\circ}$. 
Given that this is an extrema case, it suggests a definition of $C=3$ for $72^{\circ}<\mathrm{L}_{S}<108^{\circ}$. Otherwise, $\mathrm{C}=0$.

In addition to the latitude, the effective reflectance of a pixel is a useful parameter. That is to say, we also set a threshold Lambert Albedo $\left(A_{L}\right)$ that must be exceeded for a pixel to be flagged as potential surface ice. The albedo of outliers, as determined directly from the observed radiance $\left(A_{L}=(I / F) /(\cos i)\right)$, is generally $\gtrsim 0.12-0.15$; whereas that of the more diffuse structure (i.e., cloud) just south (left) and north (right) of the southern outliers in Figure $4-$ is $\sim 0.05-0.06$ (associated $\tau_{i c e} \sim 0.1$ ). For automated processing and image binning, a more conservative threshold of 0.04-0.06 would be recommended. However, for maximum flexibility, we create a set of flags which indicate a potential surface ice detection for a family of threshold values, e.g., 0.04, and 0.06, and 0.08, For the results presented in this paper, we adopt a conservative threshold of 0.04 to minimize the contamination of the results with surface ice. However, we emphasize that our methodology only creates a mask or a flag; any water ice clouds in the polar region (above a non-icy surface) are still retrieved and tabulated.

\subsection{Look-up Tables and Interpolation}

The use of a LUT is motivated by the significant increase in performance compared to that for "live" or direct calls to a multiple-scattering radiative transfer (RT) routine. A single MARCI UV image (one orbital swath) typically contains more than 300,000 pixels of valid data (i.e., photometric angles within acceptable range, etc.). Even with a notional processing time of 0.5 second per pixel, it would take more than a day to perform the RT for a single 
image (recall that there are typically 13 images per mapping day). In contrast, the approach of LUT-and-interpolation requires less than one minute for the same number of pixels using a Fortran-based implementation (compiled with gfortran and executed on a single core of circa-2012 MacPro). Of course, the computational efficiency does come with a potential cost, namely some loss of numerical accuracy due to the linear interpolation.

The error associated with interpolation may be reduced by increasing either the sophistication of the interpolation scheme (e.g., cubic spline versus linear interpolation) or the sampling frequency of the underlying function. While the function that we wish to interpolate is six-dimensional and generally non-linear, we are not limited in how the function is sampled. After some numerical experimentation with higher order interpolation routines and non-linear sampling of each dimension of the LUT, we ultimately chose a linear interpolation scheme with an increased sampling frequency (including a non-linear grid for several axes). With modern computers and sophisticated RAM caching techniques used in UNIX-based operating systems, the additional computation cost of a larger LUT is less than that for a higher order interpolation algorithm. In other words, we increase the number of sample points for each the input variables and choose a mesh to allow for (quasi-) linear behavior of the radiance between the grid points.

A general goal in the above optimization of the LUT and the algorithm choice was to keep the maximum deviation between interpolated and exact results below $1 \%$. In addition, the choice of specific tessellation for a given input parameter is the direct result of the sensitivity of the model radiance to that particular parameter and the limitation of a linear algorithm to represent 
Table 2: Water Ice Retrieval LUT Content

\begin{tabular}{llll}
\hline \multicolumn{1}{c}{ Parameter } & Number of Points & Range of Values & Mesh \\
\hline Emergence angle $(\mathrm{e})$ & 15 & $0^{\circ}-70^{\circ}$ & linear \\
Incidence angle $(\mathrm{i})$ & 9 & $0^{\circ}-80^{\circ}$ & linear in cosine of angle \\
Azimuth angle $(\phi)$ & 31 & $0^{\circ}-180^{\circ}$ & linear \\
Hapke w & 8 & $0.05-0.12$ & linear \\
Surface pressure $\left(P_{\text {surf }}\right)$ & 7 & $0.3-13.3($ mbar $)$ & linear \\
Dust optical depth $\left(\tau_{d}\right)$ & 10 & $0.01-3.01$ & non-linear \\
Ice optical depth $\left(\tau_{i}\right)$ & 23 & $0.0-4.0$ & linear \\
\hline
\end{tabular}

${ }^{\mathrm{a}} \tau_{d}$ nodes: $0.01,0.10,0.25,0.50,0.80,1.20,1.65,2.11,2.56,3.01$

that functional dependence. For example, one can demonstrate that a coarser sampling is possible for surface pressure or surface reflectance as compared to photometric angles. In general, a linear mesh tends to work well, but we are able to gain some savings in LUT size by using a non-linear sampling for the dust optical depth; bringing the size from 1.4 GB to $200 \mathrm{MB}$ without any noticeable increase in interpolation error. The smaller size decreases the read-in time of the LUT by more than $70 \%$. Similar efforts for the water ice optical depth do not yield a corresponding improvement, so we stay with a conservative sampling scheme. The final parameter sampling details for the LUT are listed in Table 2.

The interpolation and sampling error associated with a given parameter mesh is quantified using a basic monte carlo experiment. Under the assumption of a uniform distribution for the photometric angles and the Hapke w 
parameter, we (randomly) sample 7 values for each of these parameters using the ranges specified in Table 2, and combine these 7 uniformly spaced values for both $\tau_{\text {dust }}$ and $\tau_{i c e}$ (ranges also from the table). After choosing a typical surface pressure of $6.1 \mathrm{mb}$, the normalized radiance (or radiance factor, I/F; see below) is calculated for this set of 117649 parameter combinations using both LUT and direct radiative transfer calculations. Each set of calculations is performed using the same atmospheric and aerosol properties. We then assessed the distribution of fractional error in radiance against our goal of $\sim 1 \%$. The result of this exercise is the selection of the ultimate LUT, which balances data volume and interpolation error. The LUT described by Table 2 is used to generate the results shown in Figure 5. 98\% of the models having an interpolation error of less than $1 \%$, while of the models have errors less than $2 \%$.

\subsection{Radiative Transfer Algorithm}

We perform the radiative transfer calculations needed to construct the LUT using the public-domain package DISORT (DIscrete ORdinate Radiative Transfer; Stamnes et al., 1988; Thomas and Stamnes, 2002). The "frontend" model atmosphere is that described by W10, which includes the $\mathrm{CO}_{2}$ specific Rayleigh scattering cross sections of Sneep and Ubachs (2005) and Ityaksov et al. (2008). For the calculations presented here, we utilize sixteen

streams ( $N=8$ ordinate pairs), and 128 terms in the Legendre polynomial expansion for the dust and water ice phase functions. This number of terms is that needed to get a good representation of each of the phase functions, as is required for the Nakajima and Tanaka intensity correction (i.e., Laszlo et al., 2010; Stamnes et al., 1988; Lin et al., 2015). 


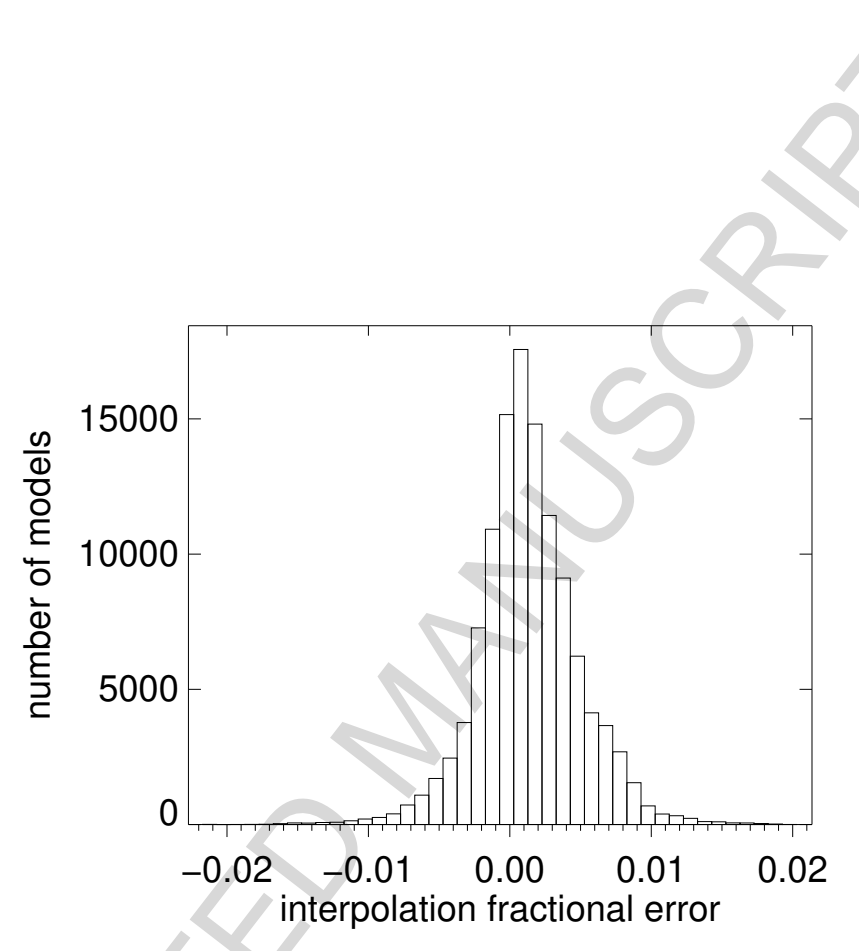

Figure 5: Histogram of the fractional error in radiance for the interpolation algorithm and LUT adopted for this study. The mean error of the 117,649 models is $0.13 \%$ and the standard deviation is $0.3 \%$. Perhaps more significantly, $98 \%$ of the models have an error less than $1 \%$ and $99.99 \%$ have an error less than $2 \%$. See text for more details. 


\subsection{Surface Reflectance Model}

Initially, we adopted the surface reflectance prescription of W10, a traditional "Hapke function" (e.g., Hapke, 1993, Chapter 12) whose parameters did not vary spatially. The limited nature of the data analyzed by W10 nearby the two Mars Exploration Rover sites and generally very dusty atmospheric conditions - may have minimized the need for a more complex treatment. However, during the early stages of the cloud mapping retrieval development, it was determined that the surface phase function needs to be more forward-scattering to avoid artifacts particularly apparent at larger phase angles (i.e., high latitudes). This type of effect concerns the five Hapke function

parameters associated with the angular distribution of the reflectance: b, c, $\bar{\theta}$, $\mathrm{B}_{0}$, and h. Using the symbol definitions of Johnson et al. (2006a,b), these are the asymmetry parameter, the backward scattering fraction, the macroscopic roughness, the opposition effect width, and the opposition effect amplitude, respectively. In addition, a second set of artifacts were noticed for images with little or no water ice opacity. The fact that this latter set of features seemed to correspond to traditional bright and dark regions suggested that the need for some spatial-variability in the model, such as in the Hapke w parameter (the single scattering albedo of the surface). We approach both issues through numerical experimentation.

Improvements in the surface phase function (i.e., set of 5 Hapke parameters) were performed through the repeated application of the cloud optical depth retrieval algorithm (as well as that for ozone, e.g., to adjust the Band 6 phase function, Clancy et al. (2016)) to regions where the surface artifacts were observed, but under conditions of minimal aerosol content. Specifically, 
we use observations at high southern latitudes during southern summer where topographic variation correlates strongly with phase angle, but also low dust and water ice columns; discrete dust events were excluded using the MARCI visible bands. In this manner, one is able to isolate the effects of the surface reflectance model. Briefly, we employ multiple overlapping images for each experiment and the requirement is that the surface phase function must produce spatially consistent $\mathrm{w}$ values. Iteration on the Hapke parameters is done manually (i.e., "chi-by-eye"), though automation is used to generate a range of candidate parameter sets to provide the analog of the Jacobian matrix for each iteration. The final parameter set is the result of approximately 10 experiments per band. The resulting more forward-scattering surface phase function is found to eliminate artifacts without introducing issues elsewhere in the dataset (e.g., at low and middle latitudes). The ultimate set of spatially-constant Hapke parameters is $\mathrm{b}=0.30, \mathrm{c}=0.70, \bar{\theta}=20^{\circ}, \mathrm{B}_{0}=1.0$, and $\mathrm{h}=0.06$ (W10 differ in their $\mathrm{c}=0.45$ and $\mathrm{B}_{0}=0.8$ ). Since our analyses using both UV bands does not clearly require a wavelength dependence, we adopt a single set of these five values; as will be seen below, spatial variability will be limited to the w parameter. The new surface phase function does result in an effective change of the mean Hapke w values of 0.07 and 0.095 in W10 for Band 6 and 7, respectively, to those of 0.057 and 0.078 . The corresponding normal Lambert $\left(A_{L}\right)$ reflectance values for the near-nadir regions become 0.014 and 0.017 , respectively (versus the previous values of 0.011 and 0.014 , respectively).

The second type of artifact suggested the need for spatially dependent Hapke w parameters The process involved in this improvement is more com- 
plicated, which we describe in Appendix A. For now, we summarize the resulting product with Figures 6 and 7 . The first figure displays the spatial dependence of the surface reflectance for Band 7. Although the map clearly contains noise at the scale of a few degrees, there are correlations of UV "bright" regional features with traditional low albedo features such as Syrtis Major, Acidalia Planitia, and Margaritifer Terra. This is a manifestation of the so-called "ultraviolet reversal" previously identified using Viking and Hubble Space Telescope observations (e.g., Thomas and Veverka, 1986; Bell and Ansty, 2007). While such efforts typically focus on smaller spatial scales, they did not include a radiative transfer-based analysis to disentangle the amplitude and spatial-extent of the effect, as illustrated in our map.

Figure 7 highlights the distribution of the $\mathrm{w}$ values and its relationship to the mean $\mathrm{w}$ value described above. The similar shape of the distribution for each of two channels is not coincidental. The intrinsically lower surface reflectance and the increased relative contribution of atmospheric scattering in Band 6 make intractable the derivation of map of similar quality to that in Figure 6. In the end, to get the Band 6 values used by Clancy et al. (2016)), we simply scale the Band 7 results by the ratio of the mean walues derived from earlier experimentation, mentioned previously.

As discussed above, we do not explicitly treat the surface reflectance of ice since the MARCI data cannot effectively discriminate between surface and atmospheric ice. As a result, a retrieval over an icy surface uses the bare surface reflectance calculated using Figure 6 and the other associated Hapke parameters. Although an effort is made to identify these cases after the fact (i.e., pixel mask), any radiance associated surface ice will be mistakenly 

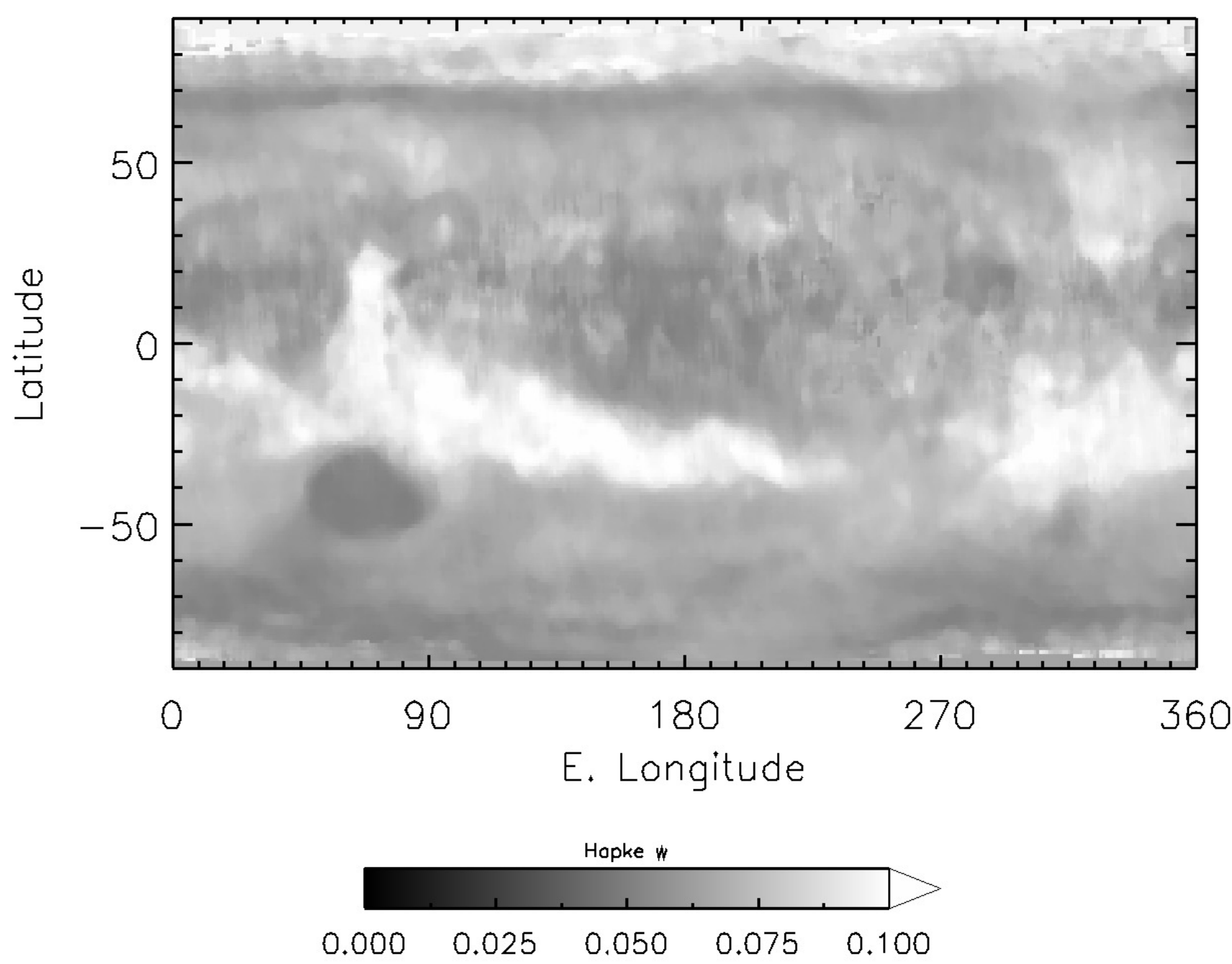

Figure 6: Map of Band 7 Hapke w. The resulting spatial dependence of the Hapke w parameter derived using the procedure discussed in Appendix A. This map provides the spatial dependence of the surface reflectance input for the ice optical depth retrieval algorithm. 


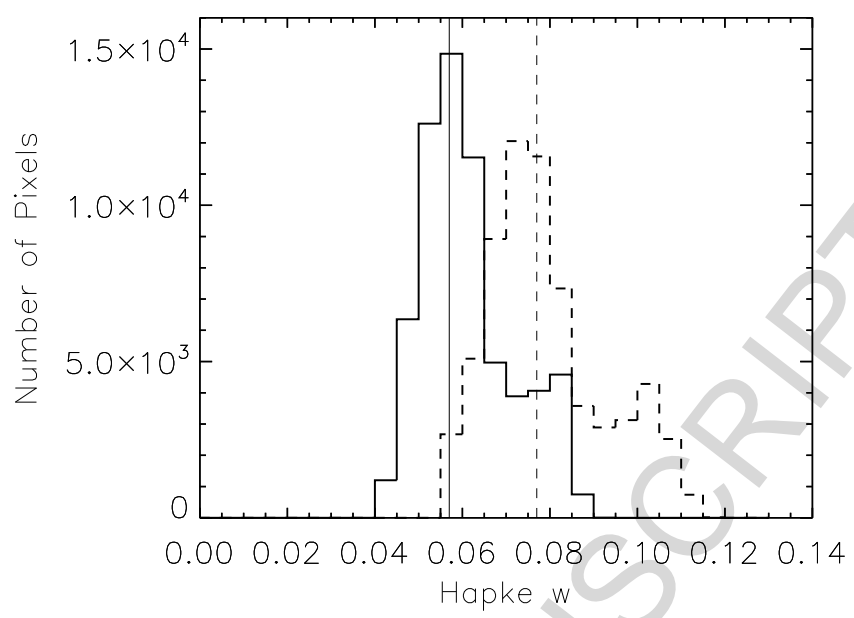

Figure 7: Histogram of the Band 6 and 7 Hapke w Maps. The distribution of w values for Band 6 (solid line) and 7 (dashed line) resulting from the process described in Appendix A. The mean Hapke $\mathrm{w}$ values in the text are shown by the vertical solid (0.057) and dashed (0.078) lines for Bands 6 and 7.

interpreted as that due to atmospheric water ice opacity.

As a closing note on this topic, our goal is to provide a function that is adequate for use as a surface boundary condition in atmospheric retrievals. Accordingly, our results require a few caveats to keep in mind. Firstly, the five surface phase function parameters (b, c, $\bar{\theta}, \mathrm{B}_{0}$, and h) are assumed to be spatially constant, with the needed variability contained in the w parameter. Anecdotal evidence for this may be found in the fact that if one re-performs the optical depth retrievals near the MER locations at low and moderate dust loading by Wolff et al. (2009), but switching the Hapke phase function parameters (i.e., MER-A becomes MER-B and vice versa), one finds optical depths and $\mathrm{w}$ values that are generally consistent (1.e., within the error bars) with those derived from the original values. Next, we assume that there is 
no seasonal component to the surface properties; namely, the w values are the same for all $\mathrm{L}_{S}$. So, temporal variability of surface features as a result of aerolian processes will introduce an error into the retrieval. Finally, we derive values with eye towards minimizing artifacts in the retrieval products that appear to be associated with the surface model. As will be seen in the error analysis presented later $(\S 4.1 .1)$, even a $20 \%$ error in the surface reflectance has a minimal effect on the retrieved optical depth. This relative insensitivity is directly due to the contrast of bright clouds with the dark nature of the surface in the UV. As a result, the effort that would be needed to derive robust surface parameter uncertainties is deemed outside the scope of this paper, and we leave such efforts for future work.

\subsection{Dust Aerosol Model}

We specify UV dust radiative properties using the model presented by W10, which is derived from MARCI UV observations during the 2007 (MY 28) global dust event. We calculate dust properties appropriate for diffuse loading conditions using a gamma distribution with moments $r_{\text {eff }}=1.5 \mu \mathrm{m}$ and $v_{\text {eff }}=0.3$ (for definitions, cf. Hansen and Travis, 1974). Following W10, the individual particle properties are calculated using the T-Matrix algorithm (e.g., Mishchenko et al., 2002) assuming a cylindrical particle with a diameter-to-length ratio $(\mathrm{D} / \mathrm{L})$ of 1 . Figure 8 shows the resulting single scattering phase functions, including the empirical correction of W10. The effective single scattering albedo values for Band 6 (260 nm) and Band 7 (320 $\mathrm{nm}$ ) are 0.64 and 0.68 , respectively. The ratio of extinction cross sections rela-

tive to $900 \mathrm{~nm}$ are: $\tau_{\text {Band6 }} / \tau_{900 \mathrm{~nm}}=0.89$ and $\tau_{\text {Band } 7} / \tau_{900 \mathrm{~nm}}=0.90$. Although Clancy et al. (2016) discuss using $r_{\text {eff }}=1.8 \mu \mathrm{m}$, this refers to the scenario of 
the refractive indices taken from W10; the same as used here. They actually use the same size distribution moments as here, i.e., a column-integrated average of $r_{e f f}=1.5 \mu \mathrm{m}$. Finally, we adopt a constant mixing ratio with respect to the gas density for the dust vertical distribution and the same size distribution for all heights.

\subsection{Water Ice Aerosol Model}

Due to the complexity associated with attempting to use spatially-variable water ice particle properties, we restrict ourselves to a single set of physical characteristics and associated radiative properties. In order to "split the difference" between properties found in the aphelion cloud belt, mid-latitudes, and (sun-lit) polar hood regions, we adopt a size distribution described by the moments $r_{\text {eff }}=3.0 \mu \mathrm{m}$ and $v_{\text {eff }}=0.1$, using a functional dependence specified by a gamma distribution. Because we are dealing with only a limited wavelength regime, the primary impact on the retrieval of different particle sizes would be seen primarily in the amplitude of the phase function in the side- and back-scattering directions. As will be discussed later ( $\S 4.1 .2)$, it is difficult to quantify the result of different particle sizes for our retrieval. The vertical profile is set using a cloud bottom of $15 \mathrm{~km}$ and a constant volume mixing ratio (with respect to gas density) above this altitude. As can be found in a literature review (e.g., Clancy et al., 2017, Table 5.1), these values are not unreasonable, particularly given the desire for simplicity (and reproducibility by others) inherent in using a single size distribution to represent a vertical column of water ice particles. Since we use only a single MARCI band (Band 7; Band 6 is included to document details cited by Clancy et al. (2016)), a mismatch between the model and the actual size distribution (for 


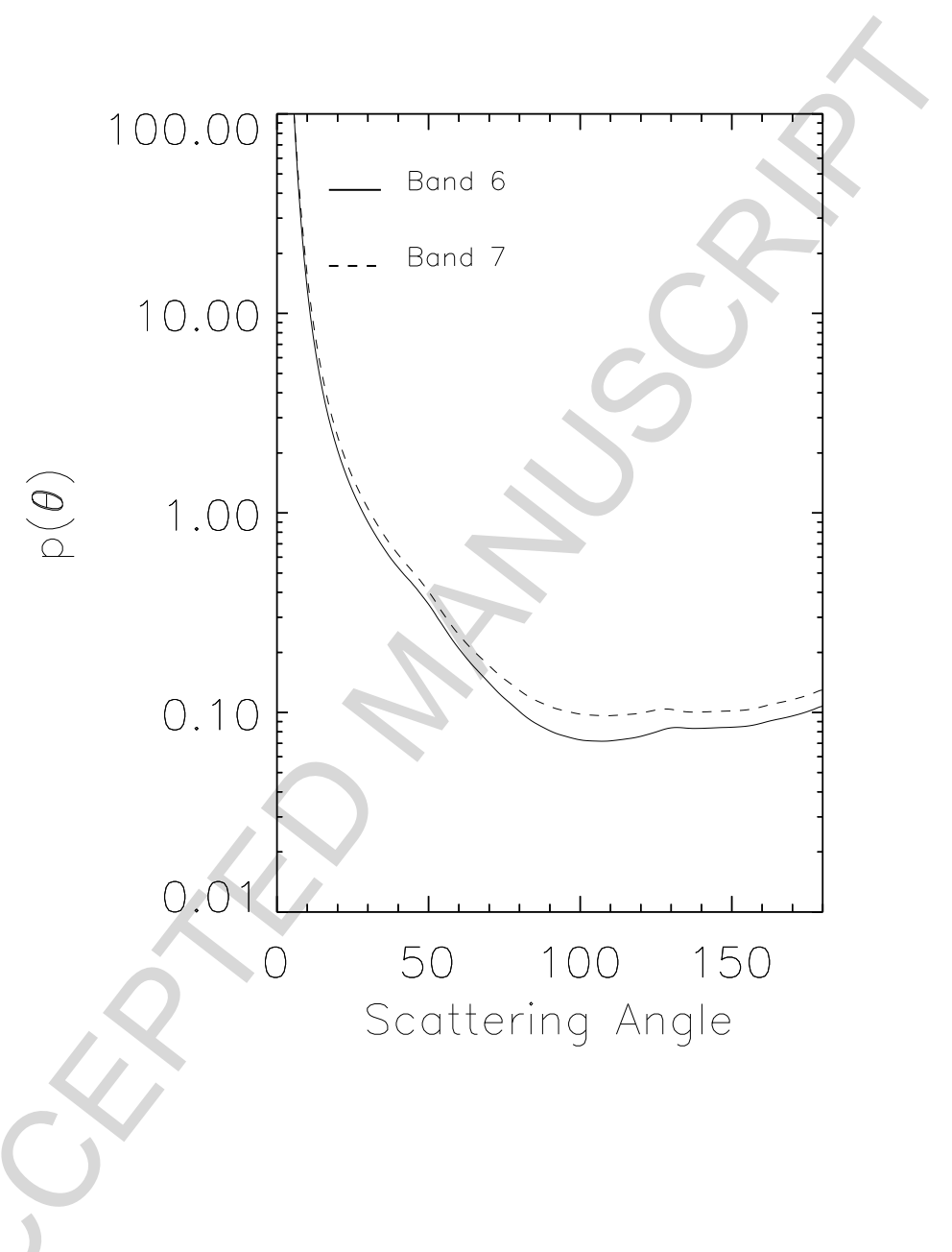

Figure 8: Adopted dust phase functions for Band 6 and 7. The T-Matrix calculations for $\mathrm{D} / \mathrm{L}=1$ cylinders (with $r_{\text {eff }}=1.5 \mu \mathrm{m}$ and $v_{\text {eff }}=0.3$ ) are modified as described by W10. See text. 
a given pixel) would impact the derived optical depth through the water ice phase function, i.e., no change in the single scattering albedo of water ice.

With a refractive index of $1.34+2.0 \times 10^{-9} i$ at $260 \mathrm{~nm}$ and of $1.33+2.0 \times$ $10^{-9} i$ at $320 \mathrm{~nm}$ (e.g., Warren and Brandt, 2008), the single scattering albedo is unity for both bands, regardless of particle shape. In terms of wavelength dependence, we use Band 7 as the reference point for the ice optical depth, but the ratio of extinction cross sections for the two bands is also essentially unity (to a precision better than $1 \%$ ).

The final radiative property needed is that of the scattering phase function. Unfortunately, Mars water ice phase function models are appreciably less mature than those for Mars dust aerosols. Crystal habits found for terrestrial cirrus particles (e.g., hexagonal prisms) have not been particularly helpful for Martian applications (e.g., Clancy et al., 2003). Fortunately, terrestrial analogs for Martian atmospheric conditions (temperature, supersaturation) suggest a more useful shape, namely an incomplete crystal habit referred to as polycrystalline or, in one realization as a "droxtal" (e.g., Thuman and Robinson, 1954; Ohtake, 1970; Baker and Lawson, 2006; Bailey and Hallett, 2009). As described in Appendix B, we adopt the droxtal shape and employ a combination of first principles scattering calculations and empirical adjustment. The resulting phase function is displayed in Figure 9, where it is compared to those for an average of the empirically derived phase phase functions by Clancy et al. (2003), for a distribution of equivalent spherical particles, and for two Henyey-Greenstein phase functions using the asymmetry parameter value of 0.7 adopted by Mateshvili et al. (2007) and that for an equivalent sphere calculation $\left(0.84, r_{\text {eff }}=3 \mu \mathrm{m}\right)$. 


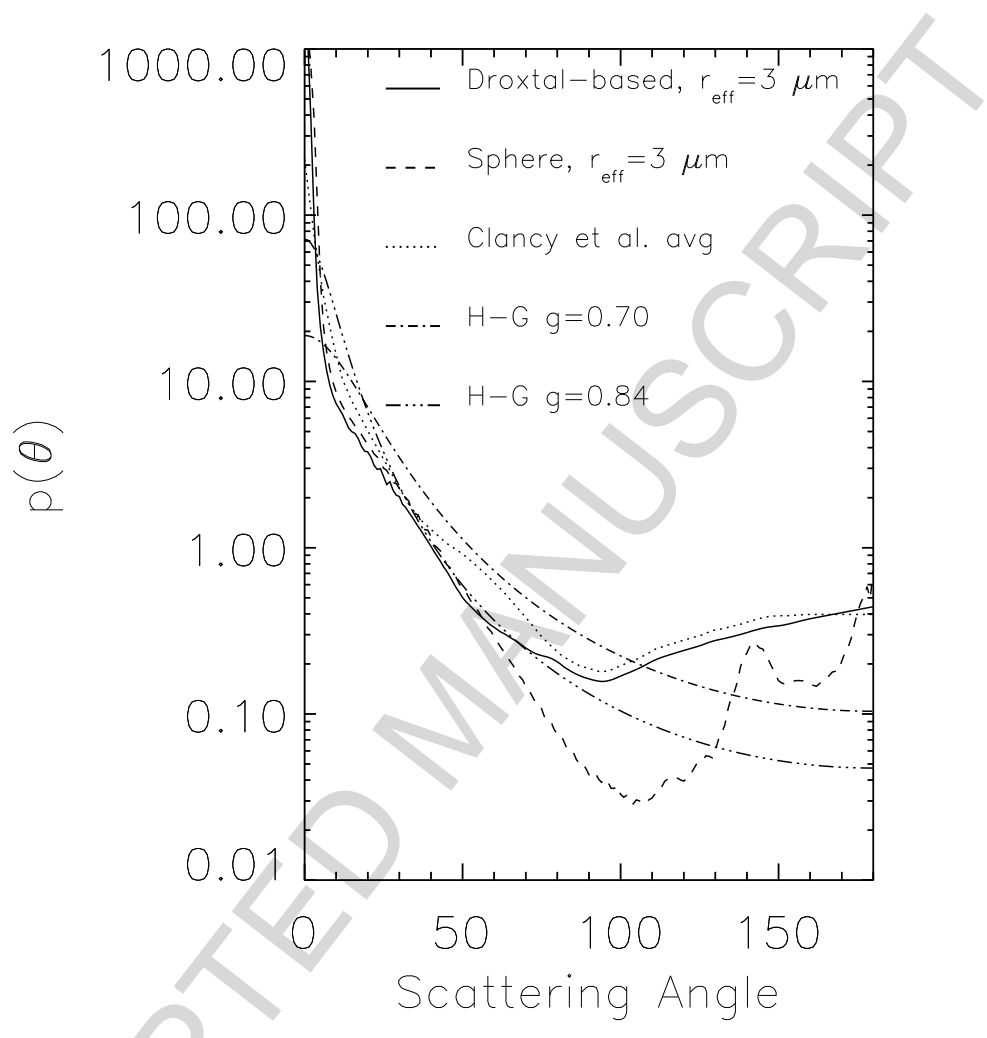

Figure 9: Adopted droxtal-based water ice aerosol phase functions for Band 7. It is compared to the phase functions for an average derived by Clancy et al. (2003, Figure 10) (one type 1, two Type 2), for a distribution of equivalent spheres, and for a HenyeyGreenstein function with asymmetry parameters of 0.7 (e.g., Mateshvili et al., 2007) and 0.84 (equivalent sphere calculation). 


\section{Retrieval Dataset}

\subsection{Water ice optical depth images and associated uncertainties}

The process described in section 3.1 produces a set of water ice optical depths in the form of an image, having the same shape and covering the same physical area as the original MARCI observation. In other words, the radiance values are replaced by the corresponding retrieval result; see the lower panel of Figure 4. The image masks (as discussed in 3.1) are also rendered in a similar format. One mask contains information about the potential inclusion of surface ice, while the other encodes information about the success (or failure) of the retrieval for that particular pixel. An advantage of retaining the image geometry/format is that the $\tau_{i c e}$ products can be easily projected onto any desired coordinate system using the geometry information associated with the original MARCI image. This is likewise true for any of the metadata values, e.g., photometric angles. An example of this capability is shown in Figure 10, where a mapping day from the aphelion cloud belt period (i.e., 01-January-2016) is projected onto a cylindrical grid.

\subsubsection{General Retrieval Uncertainties}

Excluding the possible issue of water ice particle size $\left(r_{\text {eff }}\right)$ as manifested through the water ice particle phase function (we will return to it below), the uncertainties associated with the MARCI $\tau_{i c e}$ dataset are dominated by several model input parameters and the radiometric calibration. Because the pointing registration and camera model have been validated against the MARCI observations at native $(\sim 1 \mathrm{~km})$ resolution using data taken center of the optical axis (i.e., Band 3), we treat the errors associated with the 


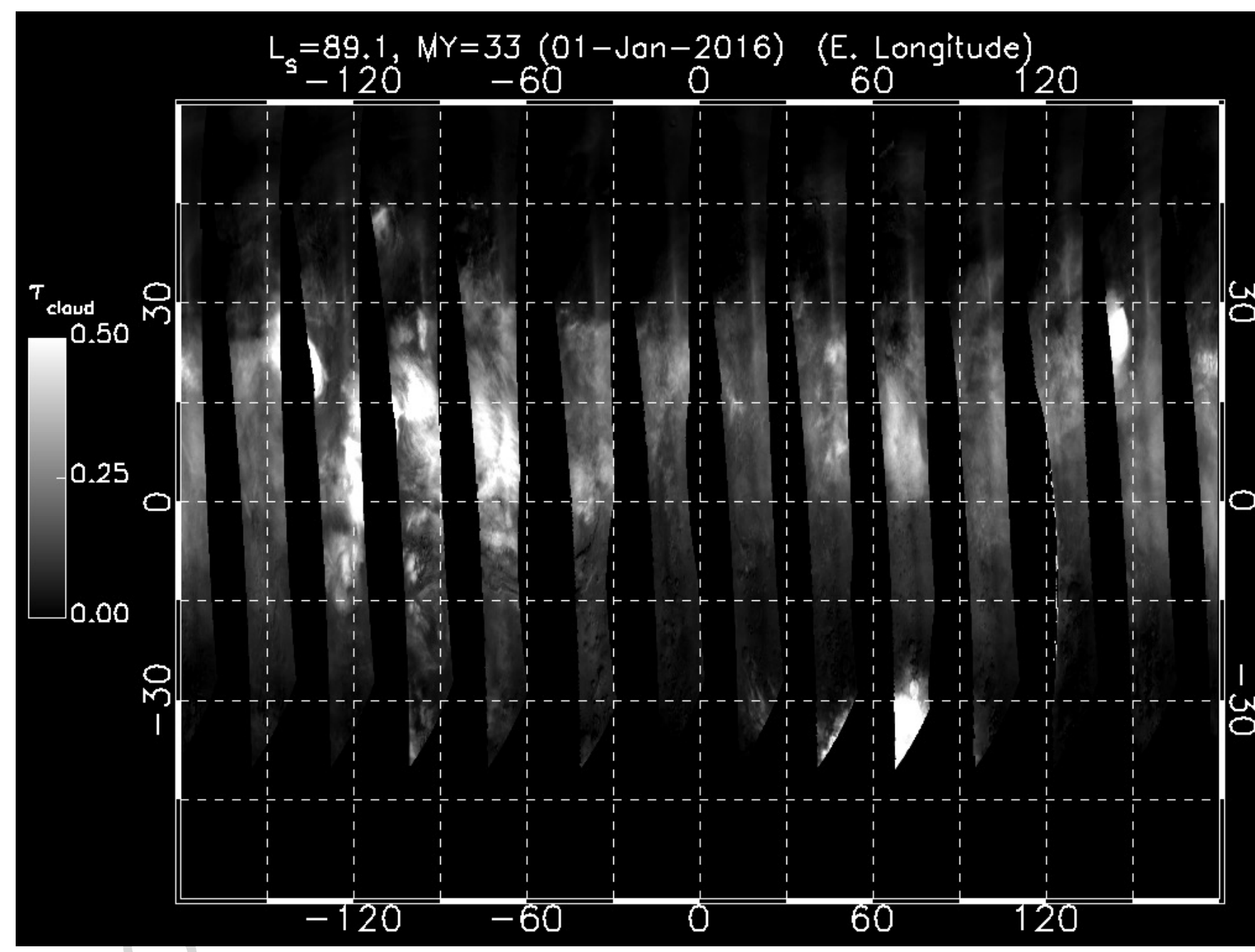

Figure 10: Map-projection of one mapping day of $\tau_{i c e}$ retrievals. The water ice cloud retrievals from 01-January-2016 $\left(\mathrm{MY}=33, \mathrm{~L}_{S}=89.1^{\circ}\right)$ are shown in a cylindrical map projection. The high latitudes are omitted to minimize surface ice confusion in this presentation. See text. 
photometric angles as negligible. We list the remaining relevant sources in Table 3. The first three rows give conservative uncertainty estimates for the specific parameters $\left(\tau_{\text {dust }}\right.$, surface pressure and surface reflectance) derived from the perturbation analysis described in the Appendix C. The first column represents an average error, while the second is a measure of the spatial variability within an image strip.

The fourth and fifth lines in Table 3 give different estimates of the total error associated with the three model inputs. The former assumes an uncorrelated behavior between the terms, which tends to capture the mean behavior. The second estimate attempts to include the effects of variability through a simple monte carlo analysis described in Appendix C. This involves representing the individual error terms as Gaussian distributions and sampling them over a comprehensive range of ice optical depths. The larger value of the monte carlo-based estimate suggests that the variability of the errors within an image does contribute to a larger total error. This is in contrast to the case of the fixed error terms in the perturbation analysis.

We also need to consider the impact of errors associated with the data. We restrict our attention to the dominant source, the radiometric calibration, and repeat the above exercises to characterize the radiometric error term and to include it in the total error estimation (see Appendix C). The results may be found on lines 6-8 of Table 3. We adopt the larger of the two errors, giving $\Delta \tau_{i c e}=0.03$ as the uncertainty of the MARCI $\tau_{i c e}$ retrievals.

\subsubsection{Retrieval Uncertainties from $r_{\text {eff }}$, Phase Function}

The uncertainty in the retrieved $\tau_{i c e}$ related the adopted $r_{\text {eff }}$ is associated with the phase functions, which is to say the difference of the model from 
Table 3: Water Ice Optical Depth $\left(\tau_{i c e}\right)$ Uncertainties (excluding ice $r_{\text {eff }}$ effects) ${ }^{\mathrm{f}}$

\begin{tabular}{|c|c|c|}
\hline Error Source & $\Delta \bar{\tau}_{i c e}$ & $\sigma_{\Delta \tau^{\mathrm{a}}}$ \\
\hline$\tau_{\text {dust }}( \pm 20 \%)$ & \pm 0.003 & 0.009 \\
\hline Hapke w $( \pm 20 \%)$ & \pm 0.010 & 0.007 \\
\hline Surface Pressure $( \pm 10 \%)$ & $+0.005,-0.009$ & .004 \\
\hline Total $_{A B O V E}(\text { uncorrelated })^{\mathrm{b}}$ & $+0.014,-0.012$ & \\
\hline Total $_{A B O V E}$ (monte carlo) ${ }^{\mathrm{c}}$ & \pm 0.019 & \\
\hline Radiance $( \pm 7 \%)$ & \pm 0.017 & \\
\hline Total $_{A L L}$ (uncorrelated) ${ }^{\mathrm{d}}$ & $+0.022,-0.021$ & $\ldots$ \\
\hline $\operatorname{Total}_{A L L}\left(\right.$ monte carlo) ${ }^{\mathrm{e}}$ & \pm 0.029 & 0.003 \\
\hline
\end{tabular}

a The standard deviation is the square-root of the mean of the variances from both perturbations.

b Error from the above three sources are added in quadrature assuming no correlation.

c Error from the above three sources, but sampled using monte carlo. See text.

${ }^{d}$ Error from all four sources are added in quadrature assuming no correlation.

${ }^{\text {e }}$ Error from all four sources, but sampled using monte carlo. See text.

f As described in 4.1.2, the retrieved $\tau$ is estimated to be $\sim 30-40 \%$ too high if the clouds have particles with $r_{\text {eff }}=1.5 \mu \mathrm{m}$, but only slightly lower (less than 10\%) for clouds with with $r_{e f f}=4-6 \mu \mathrm{m}$. 
reality. This dependence is a fundamental limitation or uncertainty inherent in scattering retrievals. As seen in Figure 9, the relative difference between various phase functions can be quite large, in particular when considering inappropriate cases (e.g., spheres, Henyey-Greenstein). For example, repeating the perturbation experiments of the previous section but this time switching the phase function, one sees relative changes that generally correlate directly with the relative differences in Figure 9. This would be expected if single scattering were dominating the observed radiance. However, before becoming too alarmed by the huge differences at some scattering angles, it is important to recall that we are comparing our model to some very inapplicable phase functions.

Of course, it is challenging to know what is more appropriate. This is particularly true for Martian water ice aerosols, which have not been examined and tested in the same way as dust aerosols (e.g., Wolff et al., 2017, and reference within). While we have endeavored to include as much physical reality in our phase function derivation (Appendix B), one must keep in mind this sensitivity to the adopted phase function. At a minimum, our efforts have yielded some degree of confidence with the shape of the water ice phase function.

Nevertheless, if we assume our model is not horribly wrong, we can estimate the impact of particle size on the MARCI optical depths. Assuming that the relative change in the opacity is dominated by single scattering effects, we can use phase function ratios. We employ droxtal calculations to calculate for $r_{\text {eff }}<3 \mu \mathrm{m}$ but need to use spheres for larger $r_{\text {eff }}$. Averaging over field-of-view effects, this simplified scenario indicates that the current 
retrieval (assuming $r_{\text {eff }}=3 \mu \mathrm{m}$ ) could over-estimate the optical depth by $\sim 30-40 \%$ if the actual $r_{\text {eff }}$ is $1.5 \mu \mathrm{m}$, while slightly under-estimating ( $\lesssim 10 \%$ ) for larger (4-6 $\mu \mathrm{m})$. Finally, unless one expects that the column-integrated particle sizes are changing from pixel-to-pixel, this type of uncertainty will tend to apply for comparisons between different regions and seasons where the microphysical processes are sufficiently different.

\subsection{Constructing a database of retrievals}

The MARCI $\tau_{i c e}$ retrieval process generates a significant amount of data, especially when one considers the months and years of data that one might wish to analyze. For example, the 1-January-2016 observations that are shown in Figure 10 produce $60 \mathrm{MB}$ of data $\left(\tau_{i c e}\right.$ and associated metadata fields), even when stored as (gzip) compressed, scaled two-byte integers. This data volume per day leads to about $220 \mathrm{~GB}$, from the beginning of mapping operations (in November 2006) until the end of September 2018. Clearly, any practical tabulation, analysis, or public data product involving multiple years of observations will require a binned product, i.e., temporally, spatially, or both.

For the purposes of the analyses and the discussions in this paper, we construct an $8 \times 8$ pixel (spatially) binned product; $\sim 1^{\circ}$ per pixel at nadir. In addition to the bin-average photometric angles, we include latitude, longitude, adopted $\tau_{d u s t}$, ephemeris time (as a proxy for Mars Year and $\mathrm{L}_{S}$ ), local true solar time (LTST; all times in this paper are LTST), surface ice pixel flag (described above), and some basic statistical information - number of original pixels in the bin (we only include retrieval pixels not flagged as possibly containing surface ice), the standard deviation about the mean for 
$\tau_{i c e}$ and $\tau_{\text {dust }}$. The size of the resulting data set - again stored as compressed two-byte integers - is about 100 times smaller: $\sim 2$ GB vs. $\sim 200$ GB. There is an additional factor-of-two savings beyond that expected from the binning due to the net decrease in metadata compared to that originally produced.

\section{Discussion}

At present, the MARCI observations span six Martian years with systematic spatial sampling, though with a limited local time coverage due to the sun-synchronous nature of the MRO orbit. For reference, the nadir LTST value for low latitudes is listed in Table 4, where the full width of the MARCI strip (assuming $65^{\circ}$ emergence angle) changes from 1.3 (Martian) hours at below $30^{\circ}$ in latitude to 2.4 hrs at $60^{\circ}$ and 2.6 hrs at $75^{\circ}$. With the large amount of data, it is natural to start with a global-scale view and then move to a more regional perspective. We begin with a zonally averaged view of the MARCI $\tau_{i c e}$ over multiple Mars Years. We then move to comparison and discussion of other observations, continuing the focus on zonal mean behavior but also including some temporally-binned zonal-meridional (i.e., latitude-longitude) products. For the latter, we limit comparison and discussion to TES data. Finally, comparisons will be made with the results from two GCMs. The theme of these discussions is to highlight the broad areas of agreement and disagreement with other observations and with model data. Such a set of exercises can potentially reveal useful information about systematic issues between data sets, including MARCI (e.g., sampling, retrieval assumptions). In addition, the inclusion of models can highlight both the successes and the potential needs for improvement. 
Table 4: Local True Solar Time at Nadir for MARCI Observations $\left(45^{\circ} \mathrm{S}-45^{\circ} \mathrm{N}\right)$

\begin{tabular}{ccc}
\hline $\mathrm{L}_{S}$ & mean LTST $^{\mathrm{a}, \mathrm{b}} \Delta$ LTST $\left._{(\text {max-min }}\right)^{\mathrm{c}, \mathrm{d}}$ \\
\hline $0^{\circ}$ & $14 \mathrm{~h} 35$ & $\mathrm{~h} 015$ \\
$90^{\circ}$ & $15 \mathrm{~h} 15$ & $0 \mathrm{~h} 15$ \\
$180^{\circ}$ & $15 \mathrm{~h} 50$ & $0 \mathrm{~h} 20$ \\
$270^{\circ}$ & $15 \mathrm{~h} 15$ & $0 \mathrm{~h} 20$ \\
\hline
\end{tabular}

${ }^{a}$ Mean LTST for emergence angles $<2^{\circ}$ at latitudes between $45^{\circ} \mathrm{S}$ and $45^{\circ} \mathrm{N}$, over MY 2834.

b The full width of the MARCI strip (assuming $65^{\circ}$ emergence angle) is about 1.3 (Martian) hours, i.e. $\pm 0 \mathrm{~h} 40$. The full width increases to $2.4 \mathrm{hrs}$ at $60^{\circ}$ latitude and to $2.6 \mathrm{hrs}$ at $75^{\circ}$.

${ }^{c}$ difference in LTST between maximum and minimum values observed for $\mathrm{L}_{S}$ over MY 2834. In comparison, the standard deviation of the LTST is always less than $40 \%$ of this difference (typically between 25-33\%).

${ }^{\mathrm{d}}$ Units are Martian hours, 24h per sol, Mars rotates $15^{\circ}$ in $1 \mathrm{~h} 00$. 


\subsection{Global-scale seasonal and interannual trends}

A zonal representation of the MARCI $\tau_{i c e}$ values, as shown in Figure 11, clearly reveals the presence and repeatability of two global-scale cloud formations: the aphelion cloud belt (ACB) and the polar hood (PH) clouds. These phenomena have been previously identified from multiple data sets and discussed by several authors, as recently summarized by Clancy et al. (2017). However, the power of the MARCI data set is to be found in the consistency of the global (spatial) coverage across the extended period of time. When displayed in this way, the general behavior of the cloud optical depths seems remarkably consistent from year to year. Nevertheless, the eye does pick out some variations in the detailed spatial extent and temporal evolution.

A simple way to look at internannual variability is to subtract the multiyear average value, which is calculated for each $\mathrm{L}_{S}$-latitude bin using a sample from all available Mars Years (for that bin). The results of such an approach can be seen in Figure 12, where a positive value represents higher optical depth at a given time than the average and a negative value less optical depth than the average. Of course, the amount of zonal averaging tends to smooth out more extreme variations that occur at specific longitudes. From this perspective, the mid-amplitude scale colors (i.e., $\left|\Delta \tau_{\text {ice }}\right| \sim 0.03$ ) can be indicative of non-trivial interannual variations. The largest variations, indicated by the saturated red and dark blue colors, are associated with the polar hoods $(\mathrm{PH})$ and the initiation/decay of the ACB.

Two other trends exist in Figure 12 that merit a brief mention. The first is the variation of cloudiness in the late northern winter season and early northern spring. The variability seems to occur at both northern and 


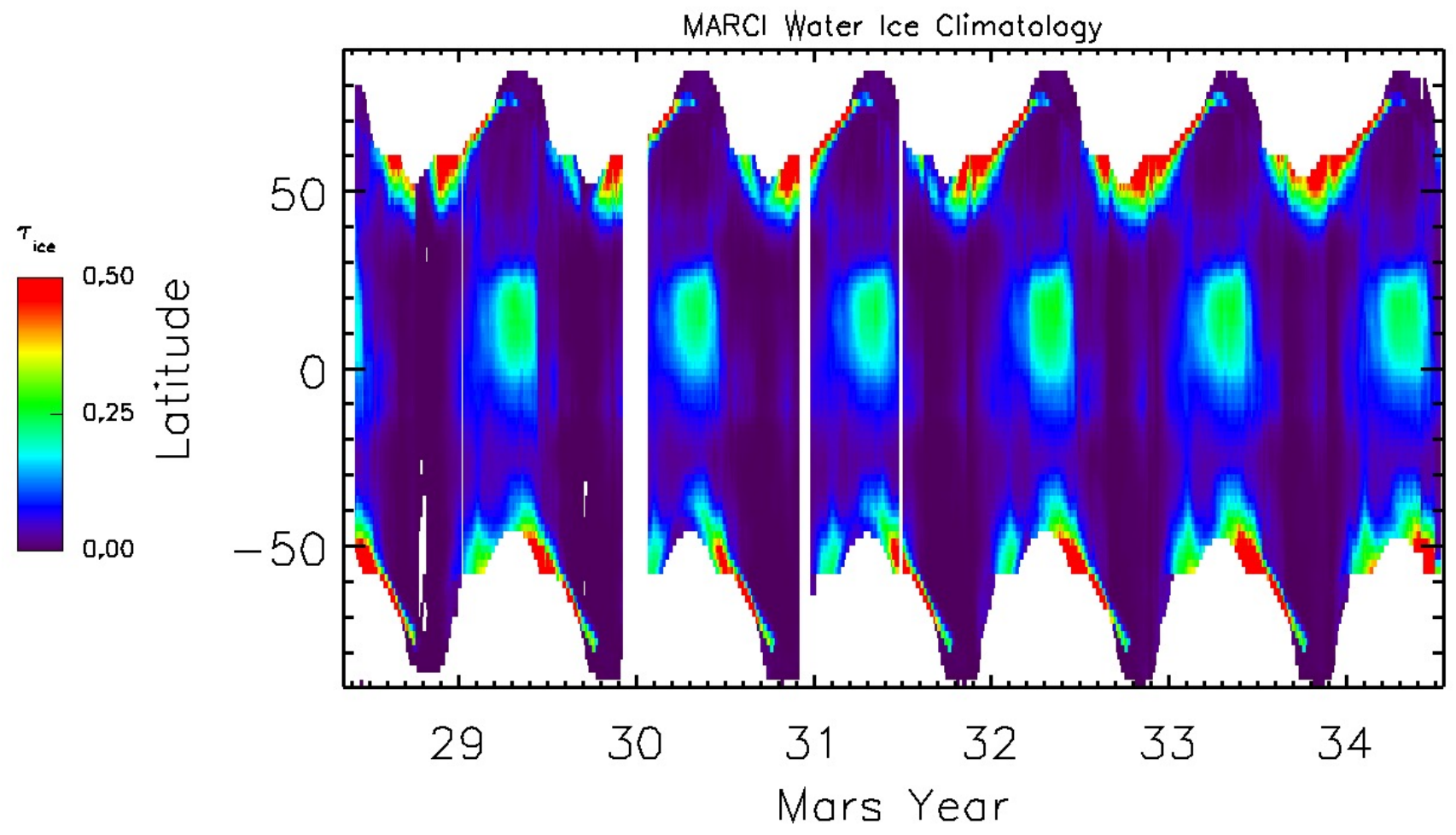

Figure 11: Zonal $\tau_{i c e}$ data set for MY $28 L_{S}=153^{\circ}$ - MY $34 L_{S}=178^{\circ}$. Each pixel represents an average of all longitudes for the given $1^{\circ}$ latitude bin. To provide the most complete temporal coverage, we also average over local times between 14h-16h LTST. Most of the polar hoods are excluded due a combination of surface ice flags and solar incidence angle constraints. 


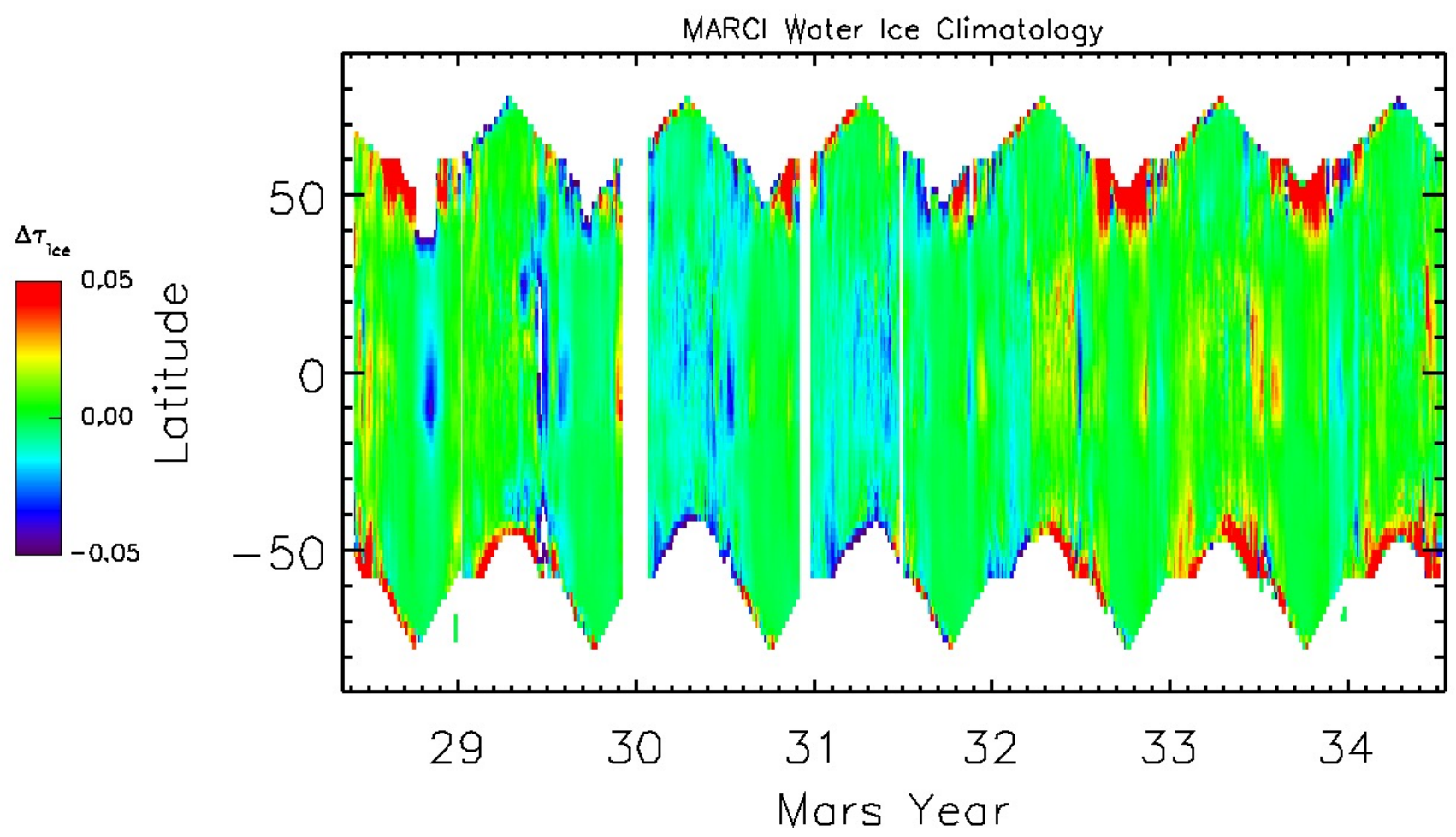

Figure 12: Zonal Difference data set for MY $28 L_{S}=153^{\circ}$ - MY $34 L_{S}=178^{\circ}$, local times included 14h00-16h00 LTST. The multi-year mean value for each $\mathrm{L}_{S}$-latitude bin (calculated using the values from all MY for that bin) is subtracted from value at a given time, so positive means more clouds than average and negative fewer. 
southern latitudes. For example, there is significantly less cloud opacity at low latitudes in late MY 28 but more in late MY 29. Another example is the trend from less optical depth in early MY 30 and 31 to more in MY 31 and 32. The second trend is the general increase of optical depth at midand low-latitudes from MY 29 through MY 33 (and perhaps into MY 34) -note the general shift of negative (blue) to positive (red) differences. So even with six Mars Years of data, it is difficult to say whether this second trend is significant or if it is potentially repeatable or periodic. There are no signs of a drift in the instrumental calibration. It is noteworthy, though speculative, to remind the reader that this trend is bracketed by occurrences of global dust events in MY 28 and MY 34.

The LTST range in Figure 11 is chosen to provide relatively complete zonal-seasonal coverage; see Table 4 for image LTST range and seasonal change of nadir values. Although somewhat unanticipated because of the relative narrow window of local times, this averaging does in fact mask appreciable diurnal changes and associated interannual variations. For example, as can be seen in Figure 13, significant increases in the opacity of the ACB can occur within a one hour period from (14h30 to 15h30), but only in some Mars Years such as MY 30 and MY 31. This is in contrast to the changes between this period and the 15h30-16h00 interval, where the differences are generally more subtle and similar to those seen in Figure 11.

\subsection{Comparisons with previous observations}

Comparing observations from different instruments can be beneficial for a variety of reasons: extension of seasonal coverage (i.e., more Mars Years), interannual variability; extension of local time coverage (e.g., diurnal changes 


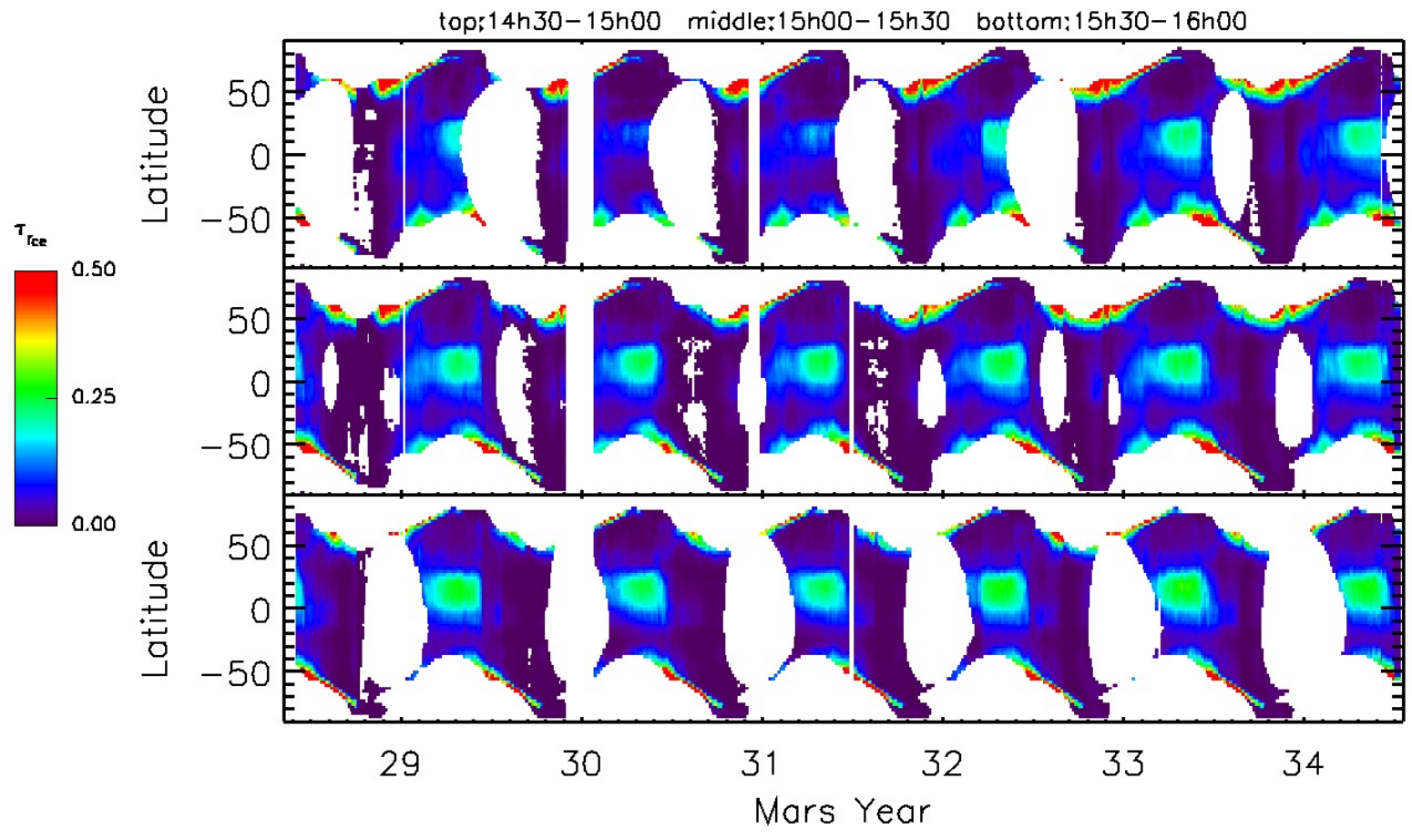

Figure 13: Local Time dependence of $\tau_{i c e}$. MY $28 L_{S}=153^{\circ}$ - MY $34 L_{S}=178^{\circ}$ for the local time intervals $14 \mathrm{~h} 30-15 \mathrm{~h} 00,15 \mathrm{~h} 00-15 \mathrm{~h} 30$, and 15h30-16h00 LTST. The changes in the completeness of the coverage for various Mars Years is due to the changes in the orbit of MRO, including maneuvers to support other missions (Menon et al., 2017). 
of aerosol loading); verification of previously phenomena and validation of retrievals; and synergies in wavelength (e.g., particle size from visible and IR) and vertical resolution (combining column-integrated values with limb profiles). In this section, we are motivated by the first few items in the list, but we restrict our attention to column-integrated optical depths from data sets with systematic spatial sampling, at least in a mean zonal sense. In addition, we focus primarily on the mid- and the low-latitude behavior, e.g., the ACB.

\subsubsection{Zonal - TES}

We begin with Figure 14, an annual zonal mean comparison using TES retrievals (from MY 24-25). The data set is described by Pearl et al. (2001) and Smith (2004). For comparison to MARCI, we use the publicly available TES water ice column-integrated absorption optical depth, which is referenced to $12.1 \mu \mathrm{m}\left(1076 \mathrm{~cm}^{-1}\right)$, kindly provided by M. Smith (personal communication). Quantitative comparisons between these TES IR absorption optical depths and MARCI $320 \mathrm{~nm}$ extinction optical depths require the assumption of an ice particle size distribution. Here we assume the same ice particle size distribution employed for the MARCI cloud retrieval LUT calculations, $r_{e f f}=3 \mu \mathrm{m}\left(v_{e f f}=0.1\right)$, In this case, the approximate ratio of the MARCI-toTES optical depth is 1.9. This number includes the cross section conversion of absorption to extinction and the scaling between $320 \mathrm{~nm}$ and $12.1 \mu \mathrm{m}$ (i.e., Clancy et al., 2003; Wolff and Clancy, 2003). We incorporate this factor in Figure 14 by scaling range of the color bar similarly (i.e., 0.50/0.26 = 1.9), such that the same color in each panel would represent the same ice column abundance under the adopted water ice particle properties $\left(r_{\text {eff }}=3 \mu \mathrm{m}\right)$. 

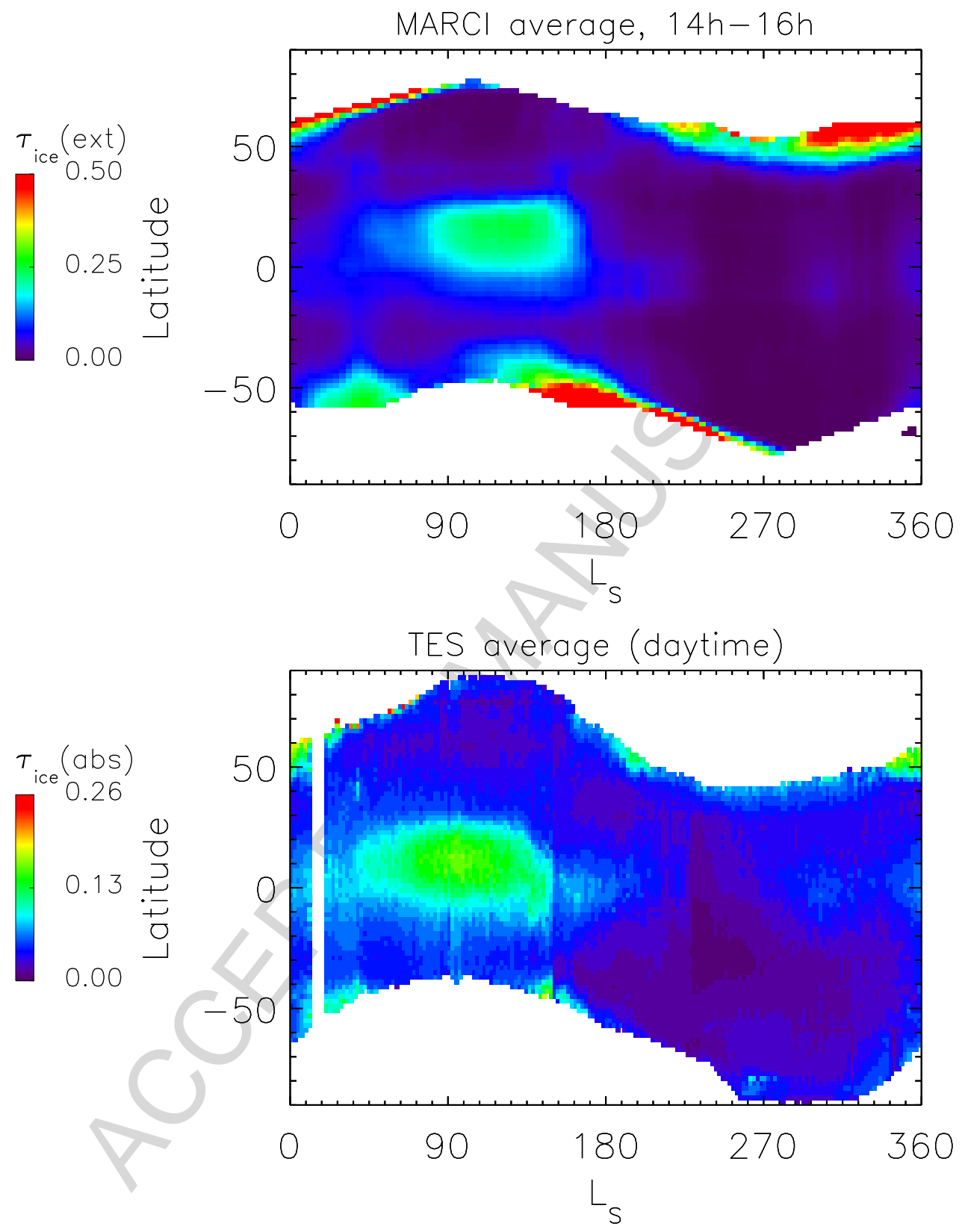

Figure 14: Multi-year mean zonal map comparison between MARCI and TES comparison. The MARCI data are extinction optical depths while those from TES are absorption optical depths. The MARCI data are averaged over the same range shown in Figure 12, both in season and local time. For TES, the average includes all valid daytime measurements (13h30-14h30 LTST). The TES "annual" data are taken from the interval that represents the highest quality data, namely MY $24 L_{S}=151^{\circ}$ - MY $25 L_{S}=151^{\circ}$ (Smith, 2004, M. Smith, personal communication). 
A quick comparison between the two panels corroborates the general correspondance of the $\mathrm{ACB}$ and $\mathrm{PH}$ features in both data sets. However, there are some visual differences worth mentioning. In the case of the polar hoods, the lack of dependence on surface temperature for MARCI allows the retrievals to extend to slightly higher latitudes than TES. On the other hand, the MARCI binning and surface ice flagging algorithm tend to remove all pixels inside the outer edge of the polar caps, including those pixels over bare surfaces. This gives TES more apparent high latitude coverage in the summer poles. So, these differences are simply a mismatch in the effective coverage between the two data sets.

A second difference is associated with structure of the ACB. From MARCI, it appears that the $\mathrm{ACB}$ is forming later in the season than observed from TES. However, taking into account the specific color scales (see above), the $\mathrm{ACB}$ in each data set actually persists for essentially the same duration, $\mathrm{L}_{S}$ $\sim 60^{\circ}-160^{\circ}$. Rather, it is the shape or profile of the ACB (versus $\mathrm{L}_{S}$ ) that is actually distinct between the two data sets. Specifically, MARCI and TES have similar ACB optical depths from $50^{\circ}-60^{\circ}$ until $75^{\circ}-80^{\circ}$ (as presented, extinction@ 320 nm vs. absorption @ $12.1 \mu \mathrm{m})$. At this point, the MARCI $\tau_{\text {ice }}$ increase appreciably and remains relatively constant until the dissipation of the ACB. In effect, the TES ACB profile is approximately symmetric about $\mathrm{L}_{S}=90^{\circ}$, while that for MARCI is skewed to later in the northern summer season having a centroid near $\mathrm{L}_{S}=120^{\circ}$ (see also Figure 28 from the GCM comparisons in $\S 5.3)$.

These $\mathrm{L}_{S}$ variations in the MARCI-to-TES optical depth ratio for the ACB potentially reflect $\mathrm{ACB}$ particle size variations over the aphelion sea- 
son. If the $\mathrm{ACB}$ after $\mathrm{L}_{S}=90^{\circ}$ manifests smaller particle sizes than assumed (i.e., $r_{e f f}=1-2 \mu \mathrm{m}$ vs. $3 \mu \mathrm{m}$ ), increased MARCI-to-TES optical depth ratios would be expected; even allowing for the overestimation of the MARCI $\tau_{i c e}$ associated with the particle size effects discussed in $\S$ 4.1.2. However, early in the northern summer season $\left(\mathrm{L}_{S} \sim 60^{\circ}-110^{\circ}\right)$, the observed TES and MARCI optical depths are consistent with the ice particle sizes $\left(r_{\text {eff }} \sim 3 \mu \mathrm{m}\right)$. We revisit these TES/MARCI ACB optical depth variations in more detail and in the context of data-GCM comparisons in section 5.3.

In addition to the potentially naive interpretation of differences optical depth ratios due to particle sizes, it is also important to consider observational coverage offsets in time (MY) and local time between the TES and MARCI data sets that might impact these TES-MARCI optical depth ratios. The TES values are compiled from a single Mars Year of observation (MY 24-25), while MARCI is an average of six Mars Years. However, the previously presented MARCI single-MY zonal means and associated interannual variations (Figures 11-12) are relatively minor and with $\mathrm{L}_{S}$ variations quite distinct from the TES/MARCI $\mathrm{L}_{S}$ variations presented in figure 15 .

The issue of the local time differences between the two data sets is another temporal distinction between the data sets $(\sim 14 \mathrm{~h}$ versus $15 \mathrm{~h}$ for MARCI). However, neither the local time dependence of the MARCI annual mean zonal $\tau_{i c e}$ shown in Figure 15 , nor the previously presented annual zonal $\tau_{\text {ice }}$ maps (Figure 13), provides observational support for interpreting the TES and the MARCI ACB L $S_{S}$-variations as consistent with local time differences. While the ACB exhibits significant diurnal variations, local time trends in the MARCI database would not suggest major ACB local time variations 


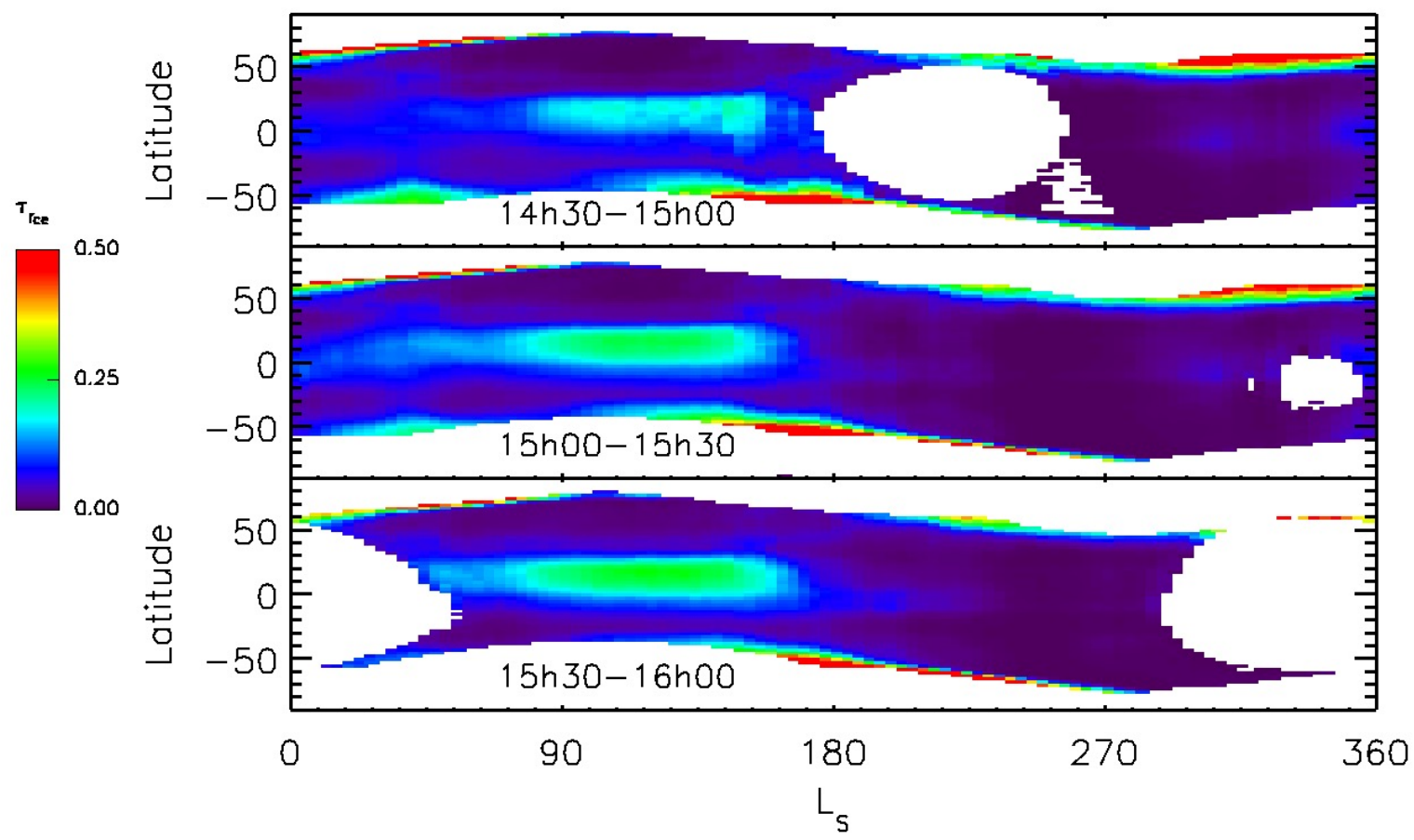

Figure 15: Local time dependence of the MARCI annual mean zonal $\tau_{i c e}$ retrievals. The mean is calculated from the data shown in Figure 13. The three LTST bins represent the bulk of the $\tau_{\text {ice }}$ data.

between the similar local times represented in the MARCI and TES data sets. This is particularly the case for the divergence in TES and MARCI ACB optical depths over $\mathrm{L}_{S}=100-150^{\circ}$. In the following section we revisit this issue in the context of THEMIS 5pm local time cloud retrievals.

\subsubsection{Zonal - THEMIS}

The THEMIS $\tau_{i c e}$ data set represents an extension of the TES data set beyond the point of spectrometer failure in 2004. A description of the retrieval algorithm and resulting data set may be found in Smith (2009). Like 
TES, the retrieval values are explicitly absorption optical depths, and referenced to $12.1 \mu \mathrm{m}$. The spatial coverage is sufficiently less systematic than that of TES such that the retrievals are generally tabulated only in a zonal mean. This data set is available by request from M. Smith (personal communication). However, since a manuscript that analyzes observations over the

lifetime of the mission (through the present) has been submitted, we restrict our discussion to the results previously published.

From Figure 6 and 8 of Smith (2009), one can see that MARCI and THEMIS data sets show a consistent behavior of the ACB structure. More specifically, each of the four MY THEMIS ACB structures (MY 26-29) is asymmetric with respect to $\mathrm{L}_{S}=90^{\circ}$, having a centroid near $\mathrm{L}_{S}=110-120^{\circ}$. In addition, a more seasonally extended (e.g., starting at earlier $\mathrm{L}_{S}$, dissipating later) ACB is seen in the THEMIS data, as would be expected from the later local times (i.e., near 17h00) and an extrapolation of the MARCI local time behavior.

\subsubsection{Zonal-SPICAM and OMEGA}

The orbit of the Mars Express spacecraft is such that the LTST at the sub-spacecraft point drifts slowly in season. As a result, characterization of an average zonal behavior over a range of local times comparable to MARCI (i.e., 2 hours) is problematic. As a result, multiple Mars Years must be combined to fill-in the gaps that would exist in any given Mars year (e.g., Figure 2, Willame et al., 2017). For water ice, systematic efforts to characterize the seasonal evolution of the zonally averaged optical depth distribution have only been published for the SPICAM instrument (Mateshvili et al., 2009; Willame et al., 2017). For OMEGA, efforts are currently ongoing with some 
initial results available in terms of the so-called "ice cloud index" (ICI; Szantai et al., 2017, 2018). The ICI, originally proposed by Langevin et al. (2007) and based upon the $3 \mu \mathrm{m}$ water ice feature, is a proxy for water ice absorption optical depth. The quantitative relationship of ICI to the optical depth is part of the current analysis effort (A. Szantai, 2018, personal communication). However, it is closely related to an absorption optical depth in the $3 \mu \mathrm{m}$ feature or a proxy for water ice mass.

Both instruments produce a mean annual zonal product that possesses an asymmetric ACB with respect to $\mathrm{L}_{S}=90^{\circ}$. For SPICAM, this can be found in Figure 8 of Willame et al. (2017), which plots extinction optical depth in the UV. If one includes data from all 4 Mars Years to provide seasonal coverage, one sees that both the amplitude and extent of the ACB structure corresponds reasonably well to that of the MARCI 14h-16h average (Figure 14). However, the specific range of local times included is not specified beyond an indication of the solar zenith (incidence) angle. For OMEGA, an averaged zonal ICI product for 14h-18h LTST is provided in Figure 1 of Szantai et al. (2018). Despite the noise and artifacts in the figure, one finds an ACB structure that approximates the $\mathrm{L}_{S}$-profile of the MARCI ACB.

\subsubsection{Zonal-Meridional-TES}

The zonal average representation is useful when looking for seasonal trends of large scale structures (or lack thereof). However, because this view tends to hide or mask interesting meridional structures and patterns, it is also of interest to compare MARCI to other observations using a longitudelatitude grid. Of the data sets considered above, only TES supports such comparisons without temporal bins that would be on the scale of the sea- 
sonal changes that one would hope to capture. As with the zonal comparison with TES, the goal is to match two data sets of column-integrated optical depths. However, it should be noted that the description of cloud spatial distributions from MOC images by Wang and Ingersoll (2002) captures the general behavior of the MARCI $\tau_{i c e}$ data associated with the ACB, polar hoods, and orographic/topographic clouds (volcanoes, Valles Marineris). As such, the reader interested in a more descriptive narrative of the temporal evolution of these features should consult Wang and Ingersoll (2002), and in particular the discussions centered around their Figures 2 and 3.

The TES atmospheric retrievals are available as a gridded product from M. Smith (see also, Smith, 2004) with spatial bins of $7.5^{\circ}$ in longitude and $3^{\circ}$ in latitude. Observations are included from Mars Years 24 through 27 (1999-2004) using temporal bins of $5^{\circ}$ in $\mathrm{L}_{S}$. Because the intrinsic meridional sampling of the TES swath or strip is 10-20 km (Smith, 2004), this $\mathrm{L}_{S}$ grid introduces additional smoothing beyond that simply associated with the spatial bin size: i.e., for any given 10 day block $\left(\sim 5^{\circ}\right.$ in $\left.\mathrm{L}_{S}\right)$ twelve or thirteen $0.2^{\circ}-0.3^{\circ}$ strips per day will not fill the meridional extent of most pixels in the grid. By comparison, $\sim 5$ mapping days of MARCI observations will provide complete longitude coverage, even with the photometric angle cut-offs associated with the radiative transfer retrievals (e.g., Figure 10).

Figures 16-27 present the MARCI-TES comparisons of water ice opacity as a function of latitude and longitude. We bin the MARCI retrievals using the same grid employed in the TES product. In order to keep the number of figures tractable, we increase the $\mathrm{L}_{S}$ bin to $30^{\circ}$ and include available data from all Mars Years in each data set. Despite the increased temporal av- 


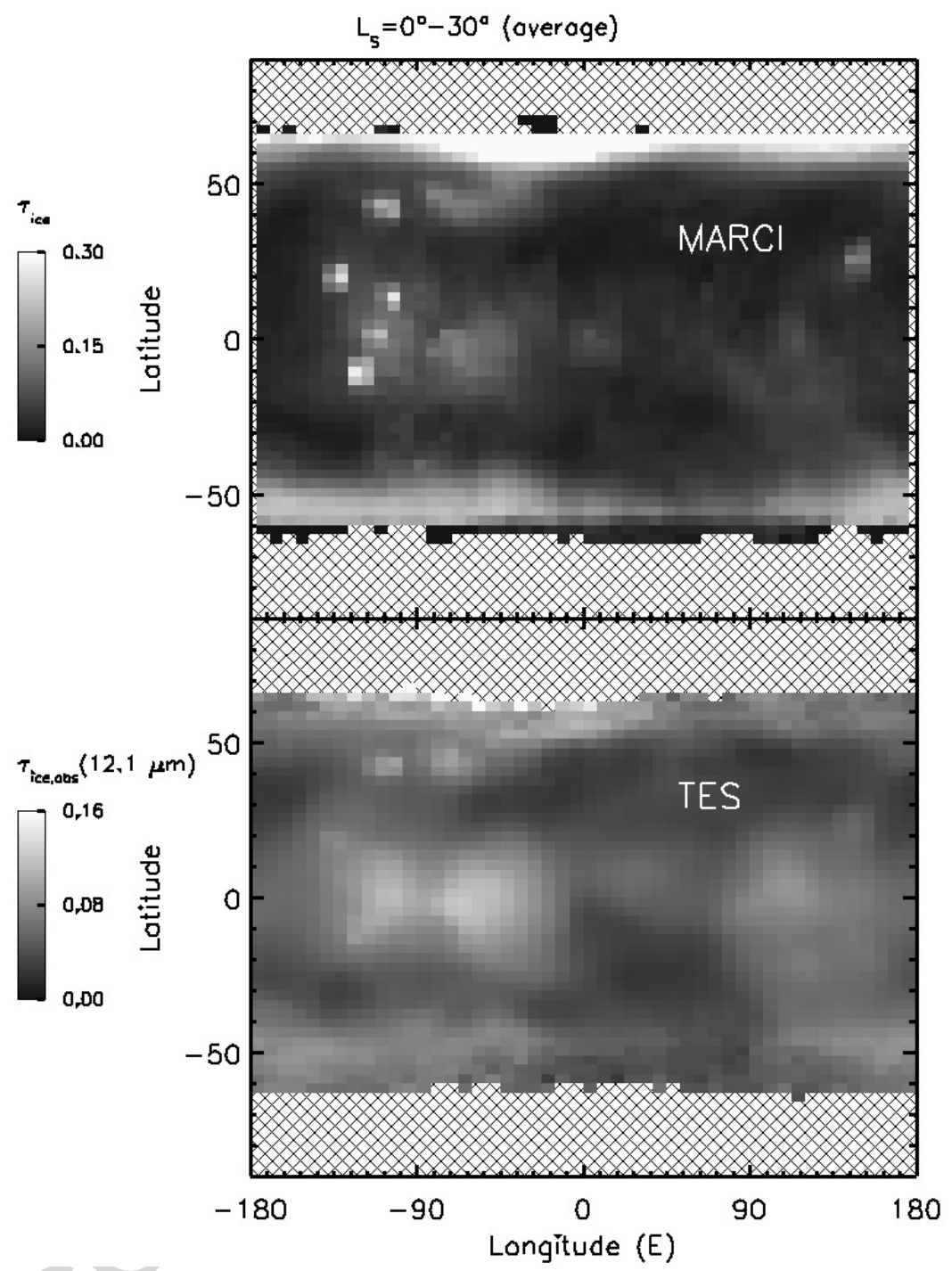

Figure 16: Comparison of the meridional behavior of water ice optical depths between MARCI (extinction) and TES (absorption) $\tau_{i c e}$, averaged over the period $L_{S}=0^{\circ}-30^{\circ}$, including all from available observations from MY 28-34 and 24-26, respectively. The TES data were provided with a binning of $7.5^{\circ} \times 3^{\circ}$, we include the full daytime local time range which is centered on 14h00 LTST. The MARCI data are binned using the same grid, and are also averaged over the 14h00-16h00 (LTST) range. The cross-hatched regions indicate the absence of valid data, (as do the black pixels - zero optical depth - surrounded on at least three sizes by cross-hatched pixels). The respective color bars are scaled by 1.9 so that the same grey level indicates equivalent 57 water ice columns, under the assumption of spherical particles with $r_{e f f}=3 \mu \mathrm{m}$. See text. 
eraging, the intrinsic smoothing of the TES values discussed above is still evident. We again scale the optical depth greyscale range by a factor of 1.9 (as discussed in $\S 5.2 .1$ ) so that the same grey level in each panel indicates an equivalent water ice column (assumingl particles with $r_{e f f}=3.0 \mu \mathrm{m}$ ), allowing for differences in the wavelength and the opacity definitions (extinction vs. absorption). 


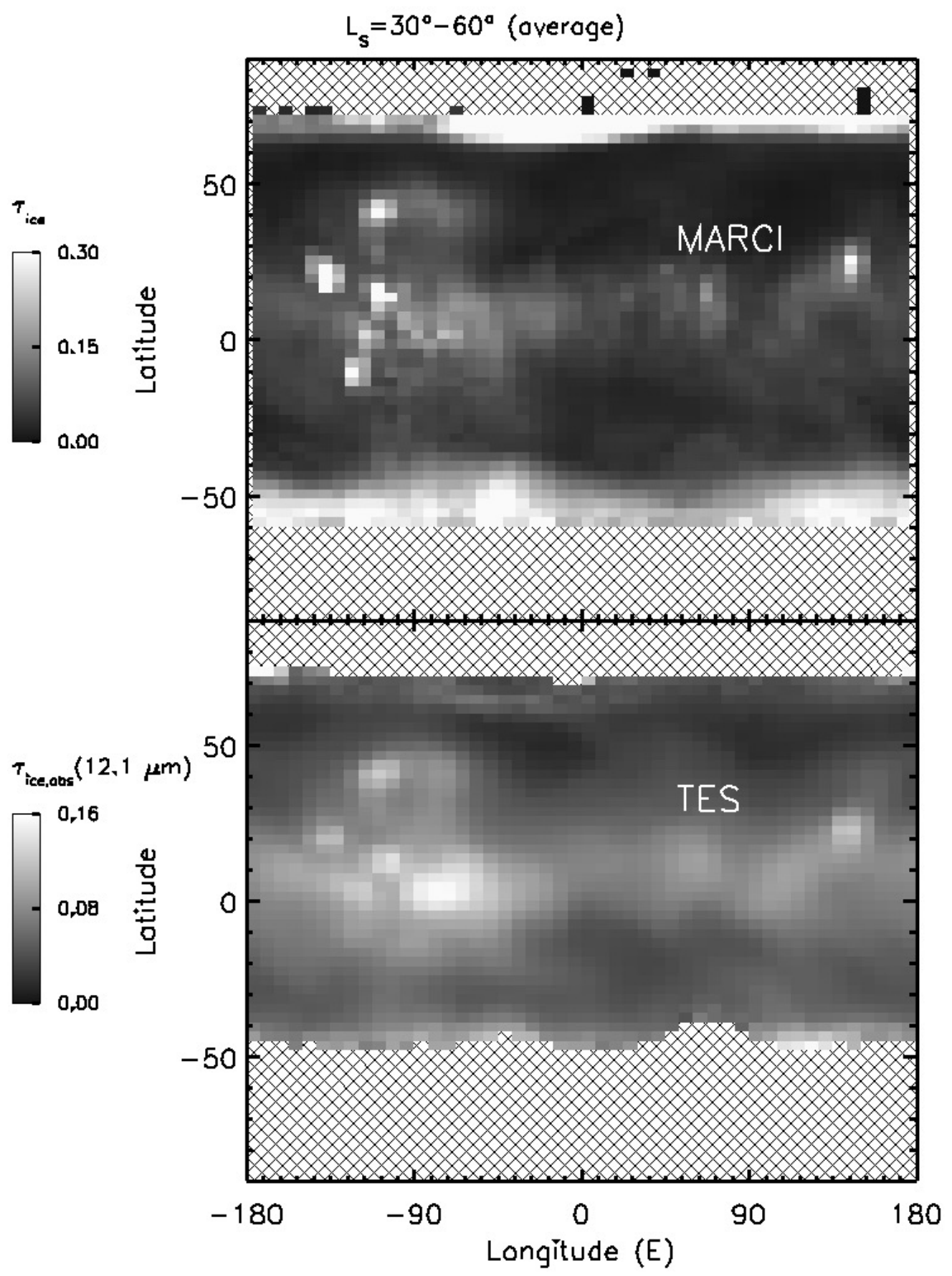

Figure 17: Same as Figure 16, but for $L_{S}=30^{\circ}-60^{\circ}$. 


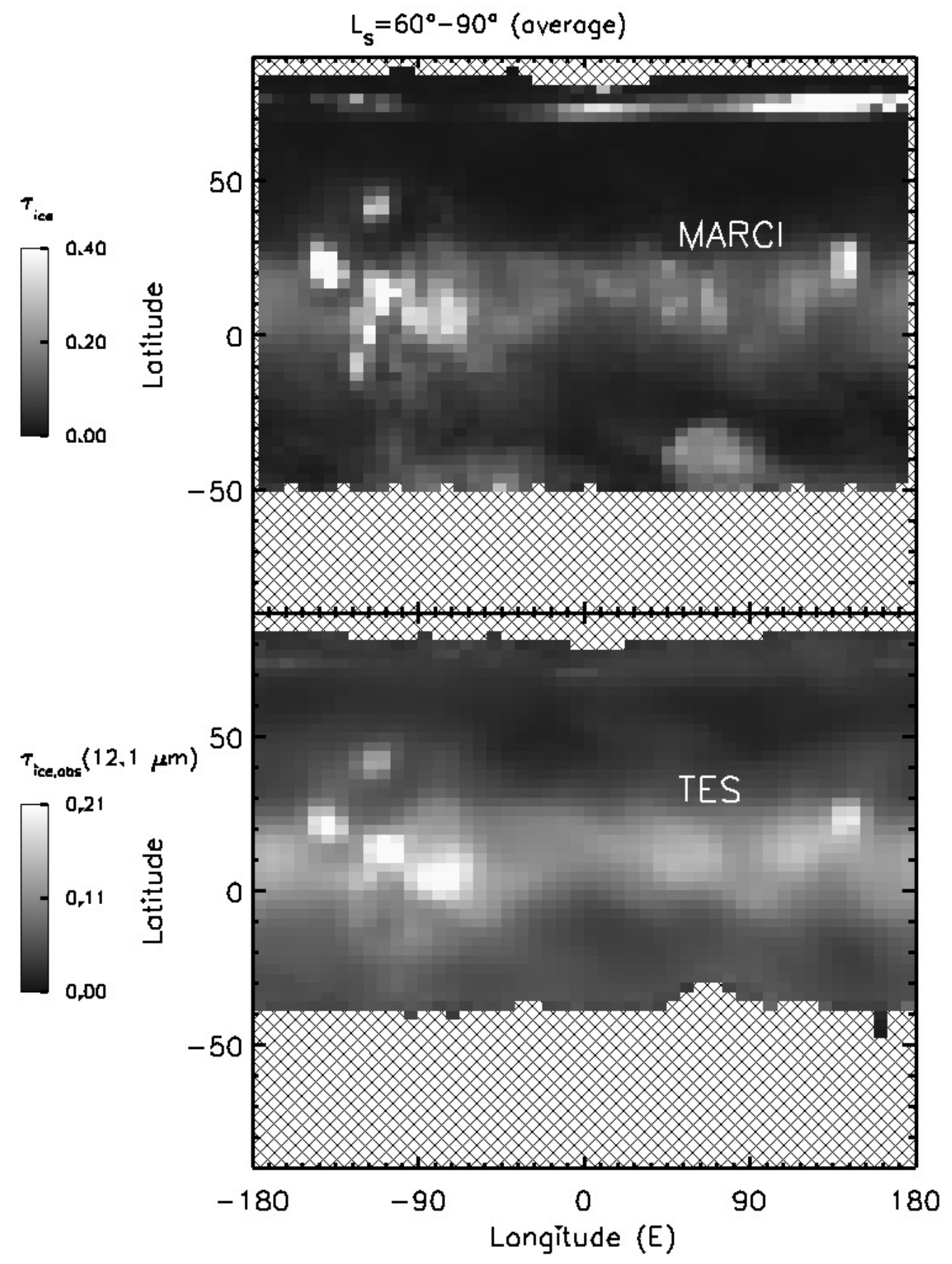

Figure 18: Same as Figure 16, but for $L_{S}=60^{\circ}-90^{\circ}$. The optical depth ranges have been increased to allow for greater optical depth associated with the northern late spring season. The large $\tau_{i c e}$ values seen by MARCI in the biogh northern latitudes are due to cap outliers that were not flagged. 
An examination of the 12 timesteps in the MARCI-TES comparison demonstrates that the general correspondance of the anticipated cloud features between the TES and MARCI retrievals is good:

- the meridional structure and evolution of the ACB correlate well, including relative changes in opacity. The asymmetric nature of the MARCI zonal results is somewhat muted by the broader temporal bins and the use of grayscale.

- the location and seasonal behavior of the orographic/topographic clouds associated with the volcanoes (including Alba Mons and Elysium Mons) and Valles Marineris are generally consistent with each other, and other observations (e.g., Wang and Ingersoll, 2002).

As with the zonal comparisons, the absence of polar hood structures in the TES opacities is anticipated because of data quality filtering associated with regions of low atmosphere-surface contrast (Smith, 2004). This can be seen through the correlation of the lower latitude boundaries of the hood in the MARCI data with those of the TES data. This includes the southern part of Hellas Basin during late southern fall and early winter, cf. Figures 18-20. There is an indication of the Hellas clouds and the polar hood in the TES data for that last figure, but the amplitude is much lower than would be expected unless the particles are very small (i.e., large MARCI-TES ratio). However, TES retrievals do detect the troughs of the north polar hood wave structure starting with the northern winter solstice (Figure 25) and continuing through the equinox where the entire southern boundary is seen (Figure 27). When the north $\mathrm{PH}$ is detected in both data sets, the meridional structures are in good agreement. 


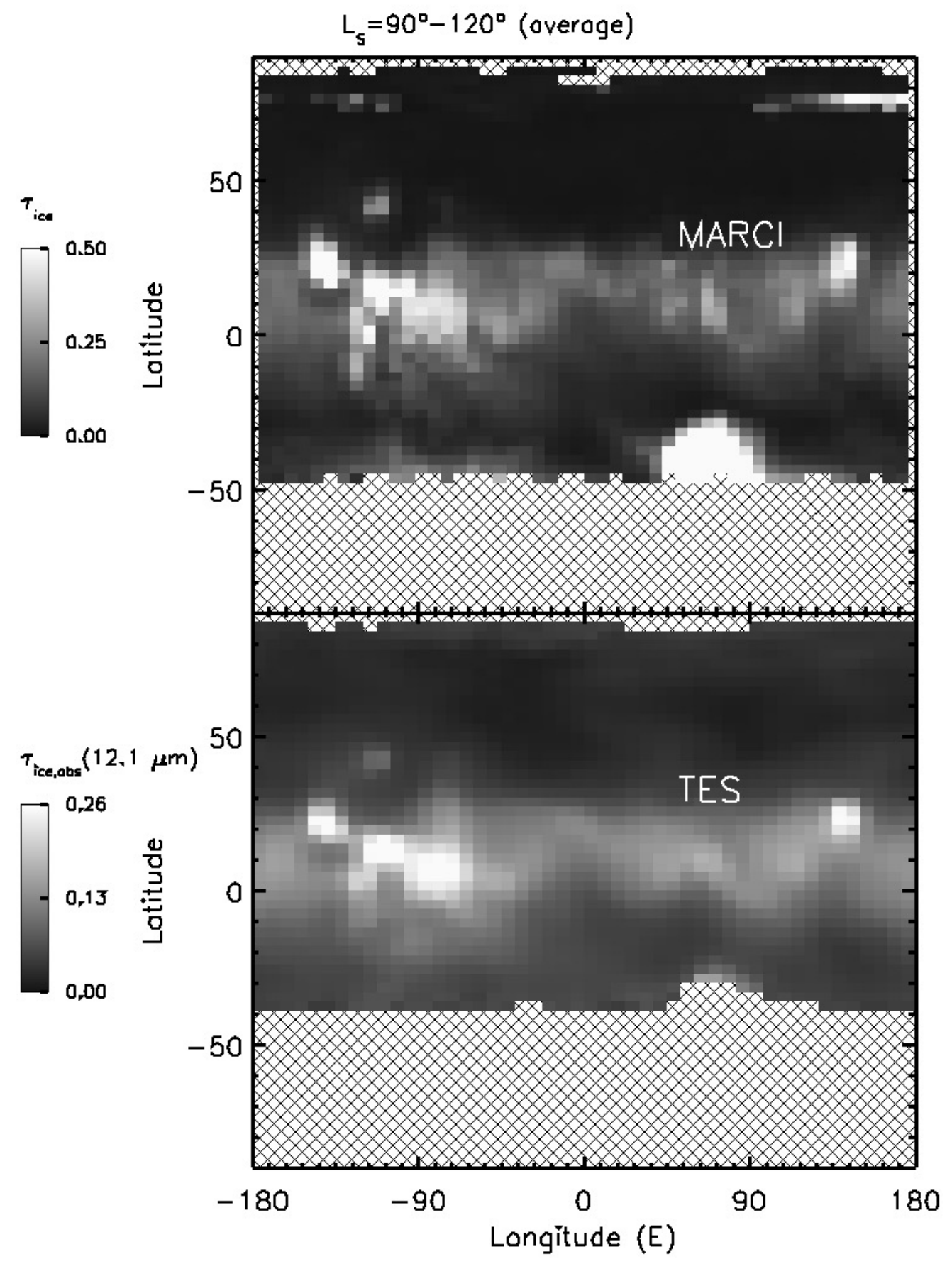

Figure 19: Same as Figure 16, but for $L_{S}=90^{\circ}-120^{\circ}$. The optical depth ranges have been increased to allow for greater optical depth associated with the northern summer season. The large $\tau_{i c e}$ values seen by MARCI in the figh northern latitudes are due to cap outliers that were not flagged. 


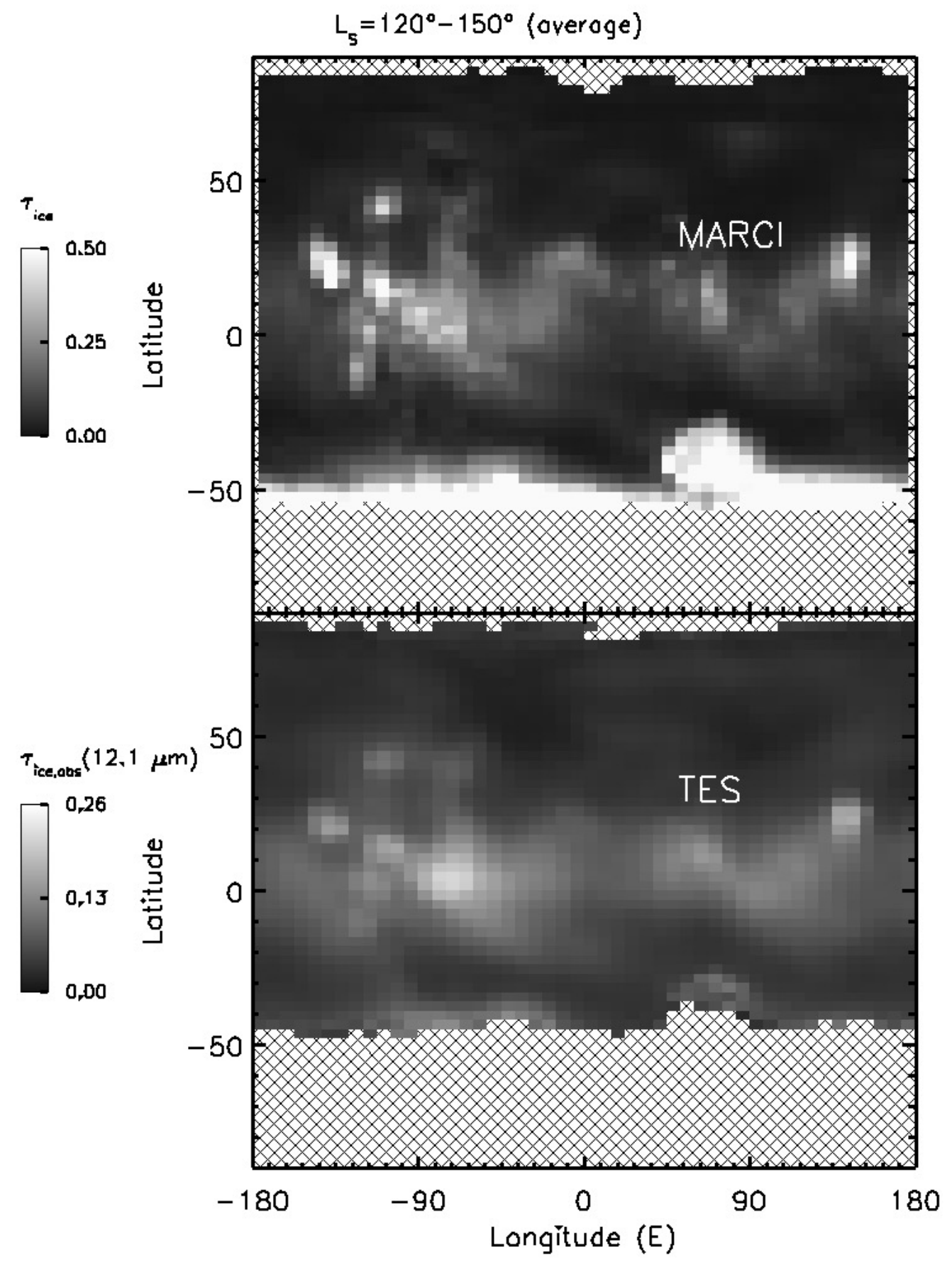

Figure 20: Same as Figure 16, but for $L_{S}=120^{\circ}-150^{\circ}$. The optical depth ranges have been increased to allow for greater optical depth associated with the northern summer season. 
There are also several cases of potential dichotomies in the MARCI-TES comparisons. These are interesting both from the perspective of the insights in terms of particle properties, but also as indicators of the difficulties and limits of these types of comparisons. Recalling that the scaling of the gray levels in these figures is designed to produce similar brightness levels for similar water ice columns with $r_{e f f}=3 \mu \mathrm{m}$. Correspondingly, brighter levels in the MARCI plot suggest smaller particles and brighter levels in TES are consistent with larger particles.

- The clouds associated with the volcanoes typically have some noticeably brighter pixels in the MARCI data, potentially indicative of smaller particle sizes. Given the smoothing possibly induced through the temporal binning of the initial gridded TES product (from M. Smith, $5^{\circ}$ in $\mathrm{L}_{S}$ ), it is reasonable to consider the potential for incomplete sampling. On the other hand, microphysical modeling of orographic clouds by Michaels et al. (2006) indicates that smaller particles can be produced under dryer conditions, such as those associated with earlier northern spring. For reference, the MARCI-TES ratio increases to 2.4 for $r_{e f f}=2 \mu \mathrm{m}$ and jumps to 5 for $r_{e f f}=1 \mu \mathrm{m}$. Any further investigation of this issue will require the use of higher resolution retrieval products for both TES (i.e., native geometry of $\sim 10 \mathrm{~km}$ strips) and MARCI ( $\sim 10 \mathrm{~km}$ pixels).

- The clouds associated with Valles Marineris also demonstrate interesting opacity ratio behavior. In this case, TES has higher opacity levels than those expected by extrapolating from MARCI using the $r_{\text {eff }}=3 \mu \mathrm{m}$. This behavior is most striking outside of the ACB period, i.e., Figures 16, 17, 21, and 22. The behavior of the MARCI clouds is consistent with that shown in Wang 
and Ingersoll (2002). With the MARCI-opacity ratio for these particular time steps in the range 1-1.5, the result is certainly consistent with larger particles. However, given that the particle size diagnostic ratio (MARCITES) effectively saturates at a lower limit of $\sim 1.5\left(r_{\text {eff }}>5 \mu \mathrm{m}\right)$, we may be seeing the effects of retrieval uncertainties, particularly for the lower $\tau_{\text {ice }}$ values.

- TES detects modest amounts of water ice in Hellas during southern spring, but in this case without a corresponding detection by MARCI, see Figures 2224; nor is this feature evident in Figure 2 of Wang and Ingersoll (2002). This apparent discrepancy may be due to the simple prescription employed for the vertical distributions of water ice and dust in the MARCI retrieval algorithm (see $\S 3.5$ and 3.6). If the water ice clouds were near the surface at the bottom of Hellas and most of the dust column (which is moderate-to-large at this season) above these clouds, the water ice could be effectively hidden from MARCI (and MOC) photometry while remaining detectable by TES though the $12.1 \mu \mathrm{m}$ spectral signature.

- Finally, the TES retrievals contain diffuse water ice opacity that has no obvious counterparts in the MARCI maps. It is particularly evident for the period $\mathrm{L}_{S}=300^{\circ}-30^{\circ}$ (Figures 26, 27, and 16). The TES $\tau_{i c e}$ are not insignificant, with values in the range $0.05-0.08$. 


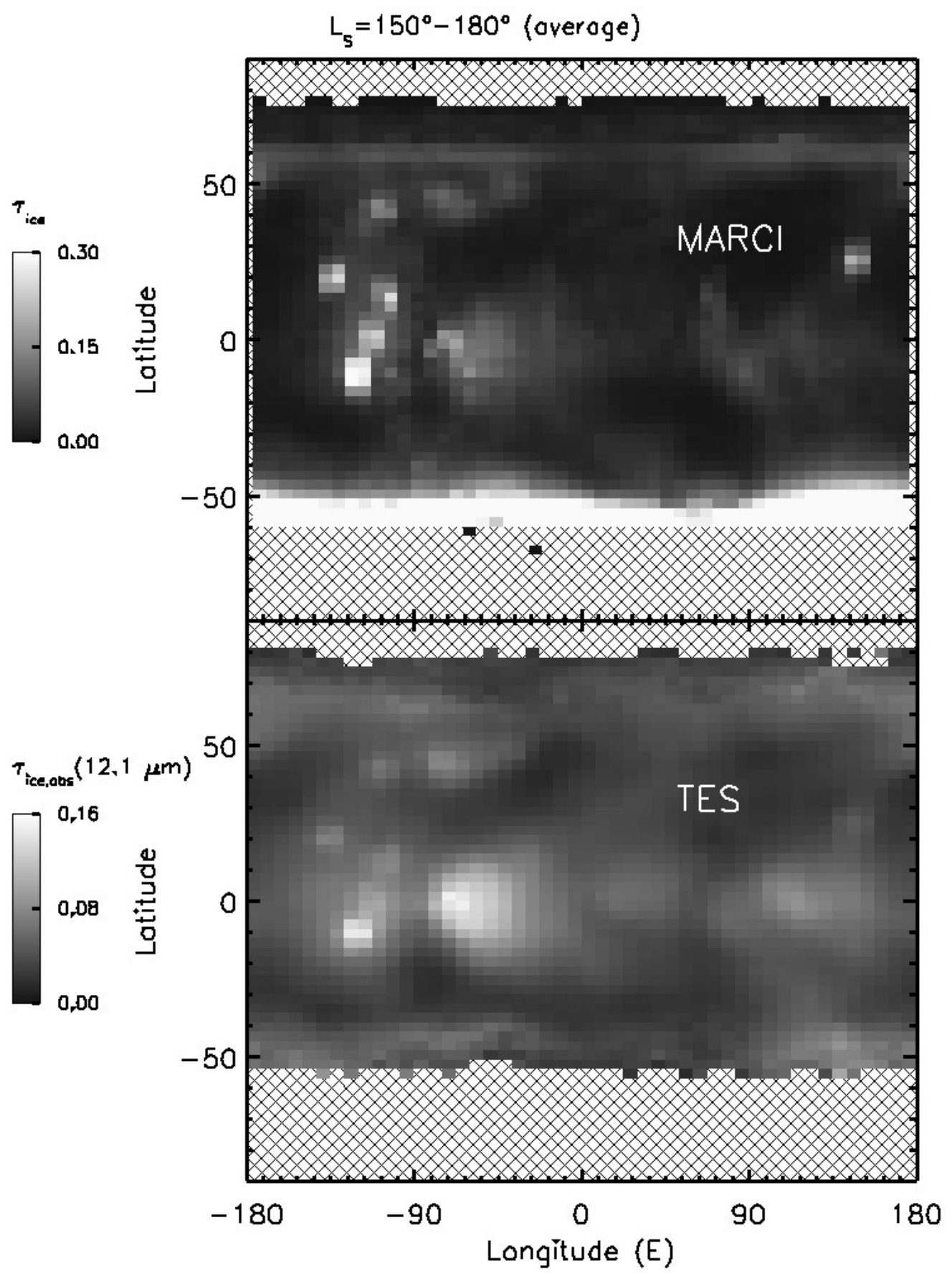

Figure 21: Same as Figure 16, but for $L_{S}=150^{\circ}-180^{\circ}$. 


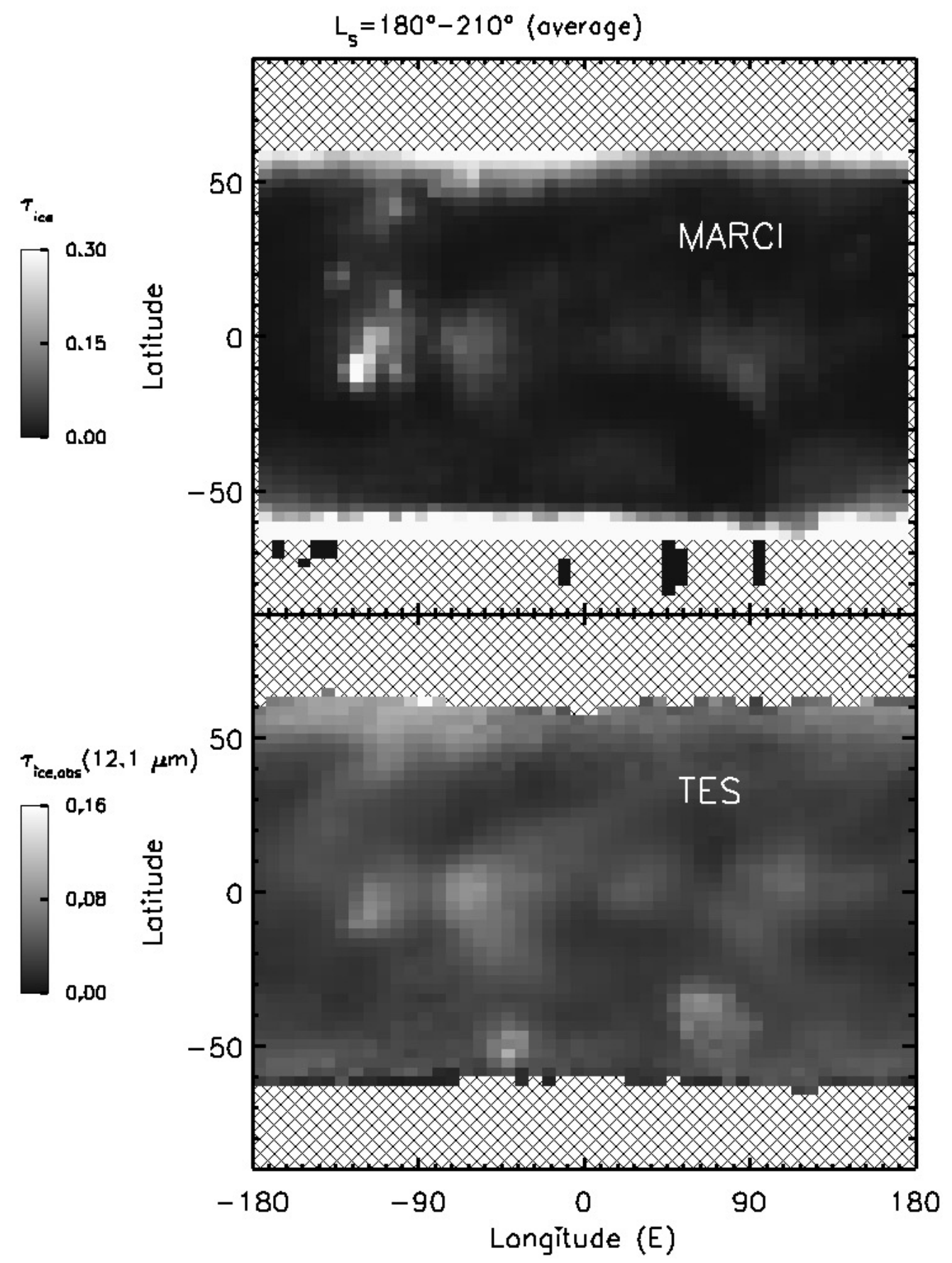

Figure 22: Same as Figure 16, but for $L_{S}=180^{\circ}-210^{\circ}$. 


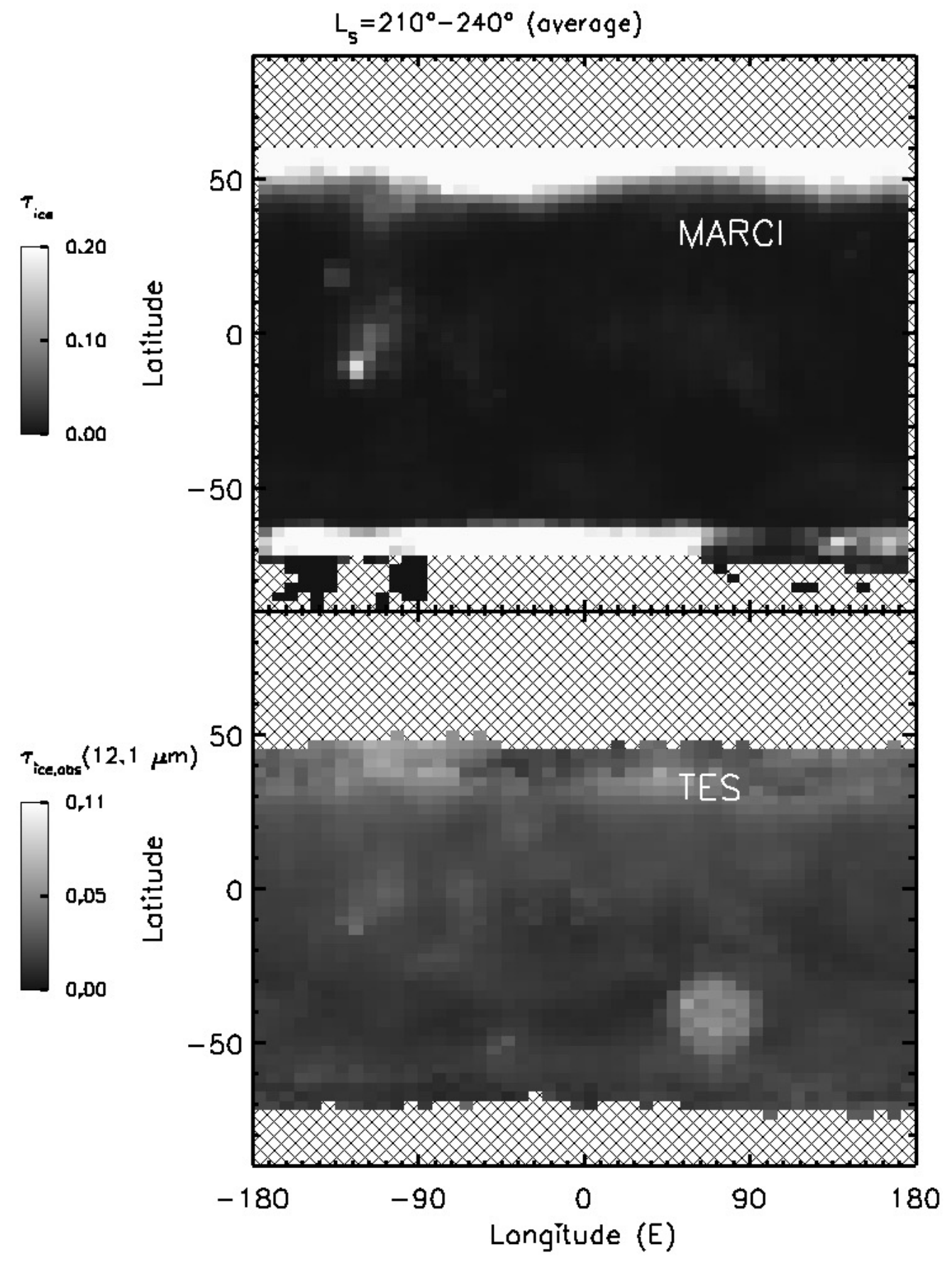

Figure 23: Same as Figure 16, but for $L_{S}=210^{\circ}-240^{\circ}$. The optical depth ranges have been decreased to allow for lower optical depth associated with the southern spring season. 


\subsection{Comparison with Global Climate Models}

A primary motivation in the development of the MARCI $\tau_{i c e}$ retrieval has been the ability to generate products for comparison to and, perhaps optimistically for assimilation by Mars GCMs (MGCM). Significant advances in the capabilities of MGCMs to capture the spatial and temporal evolution of water ice in the Martian atmosphere have occurred over the fifteen years since the initial reproduction of the ACB structure by Richardson et al. (2002). Thus, we compare MARCI zonal cross-sections and three latitude-longitude snapshots with the simulated cloud optical depths from two MGCMs. The MARCI data are chosen to provide examples of the types of structures already examined: ACB, polar hood, and topographic-orographic clouds.

The MGCM output is generated by models from the NASA Ames Research Center (Ames) and from the Laboratoire de Météorologie Dynamique (LMD). Both models have been well described in the literature, with the particular data sets that we are using described and documented by Haberle et al. (2018a) and Pottier et al. (2017), respectively. These models are optimized to reproduce the observed water cycle on Mars, including the microphysical formation and transport/dynamical impact of water ice clouds. For more description of the models, the reader is referred to Navarro et al. (2014, and references within) for the LMD GCM, and Haberle et al. (2018a) for the Ames GCM. Both models calculate ice optical depths as a function of simulated ice particle size distributions and number densities. The LMD GCM routinely generates $12.1 \mu \mathrm{m}$ absorption optical depths (i.e., TES $\tau_{\text {ice }}$ ). The Ames model provides two optical depths, a shortwave UV band extinction optical depth (effectively, the MARCI $\tau_{i c e}$ ), as well as the same IR 


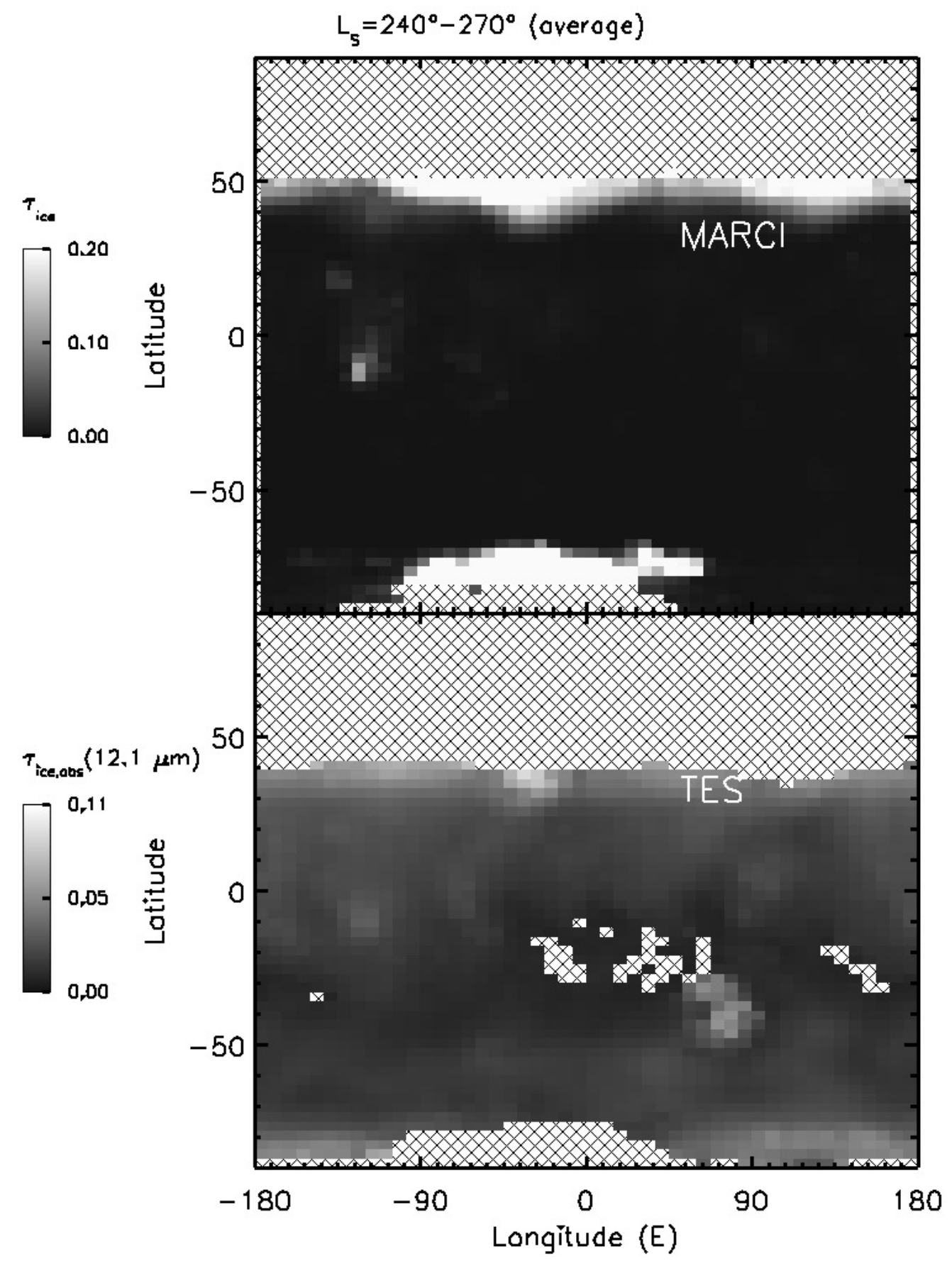

Figure 24: Same as Figure 16, but for $L_{S}=240^{\circ}-270^{\circ}$. The optical depth ranges have been decreased to allow for lower optical depth associated with the southern spring season. The large $\tau_{i c e}$ values seen by MARCI in the south0polar region are parts of the cap the surface ice flag algorithm. 


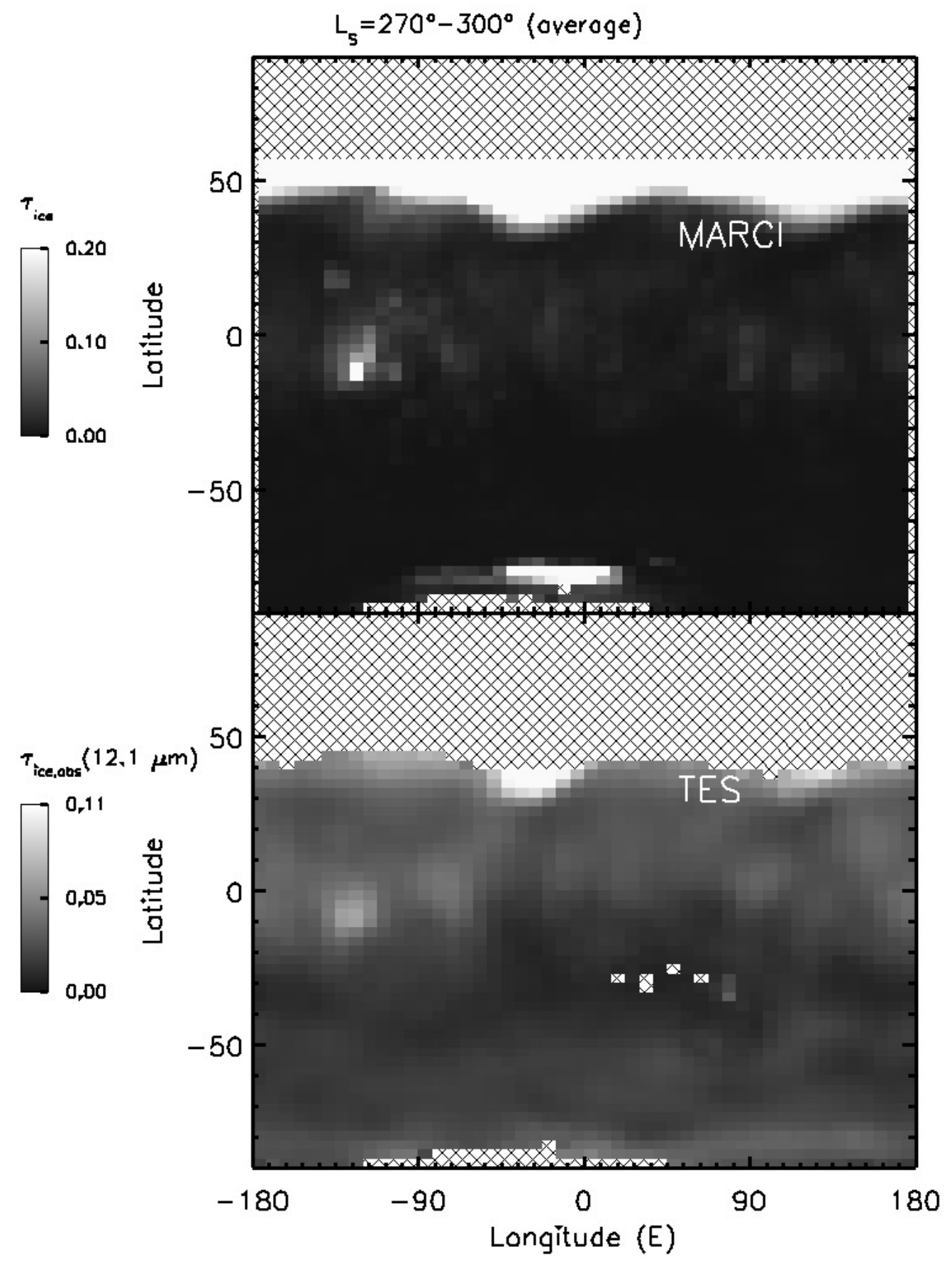

Figure 25: Same as Figure 16, but for $L_{S}=270^{\circ}-300^{\circ}$. The optical depth ranges have been decreased to allow for lower optical depth associated with the southern summer season. The large $\tau_{\text {ice }}$ seen by MARCI in the south $p$ blar region is again surface ice missed by the surface ice algorithm. 


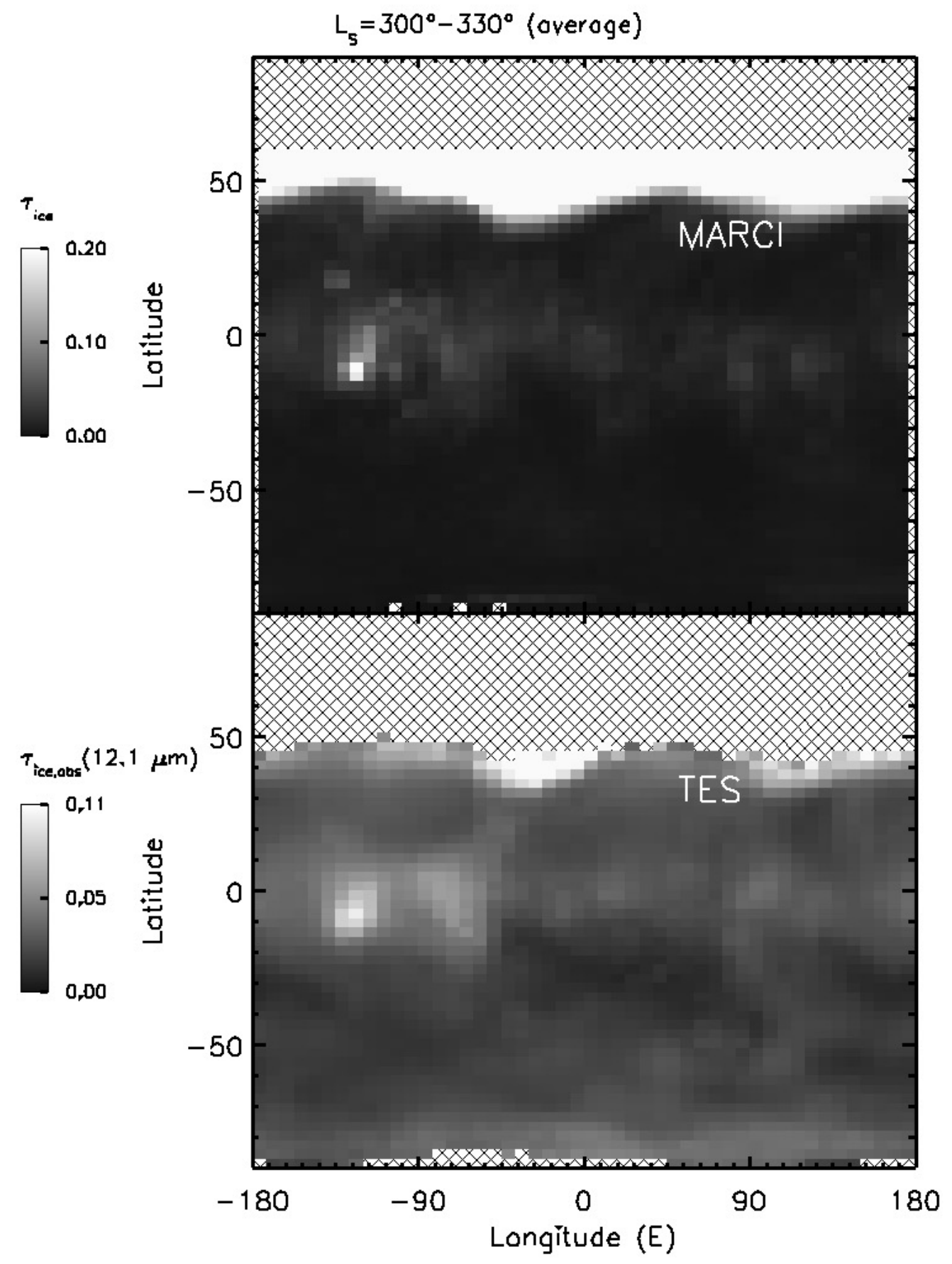

Figure 26: Same as Figure 16, but for $L_{S}=300^{\circ}-330^{\circ}$. The optical depth ranges have been decreased to allow for lower optical depth associated with the southern summer season. 


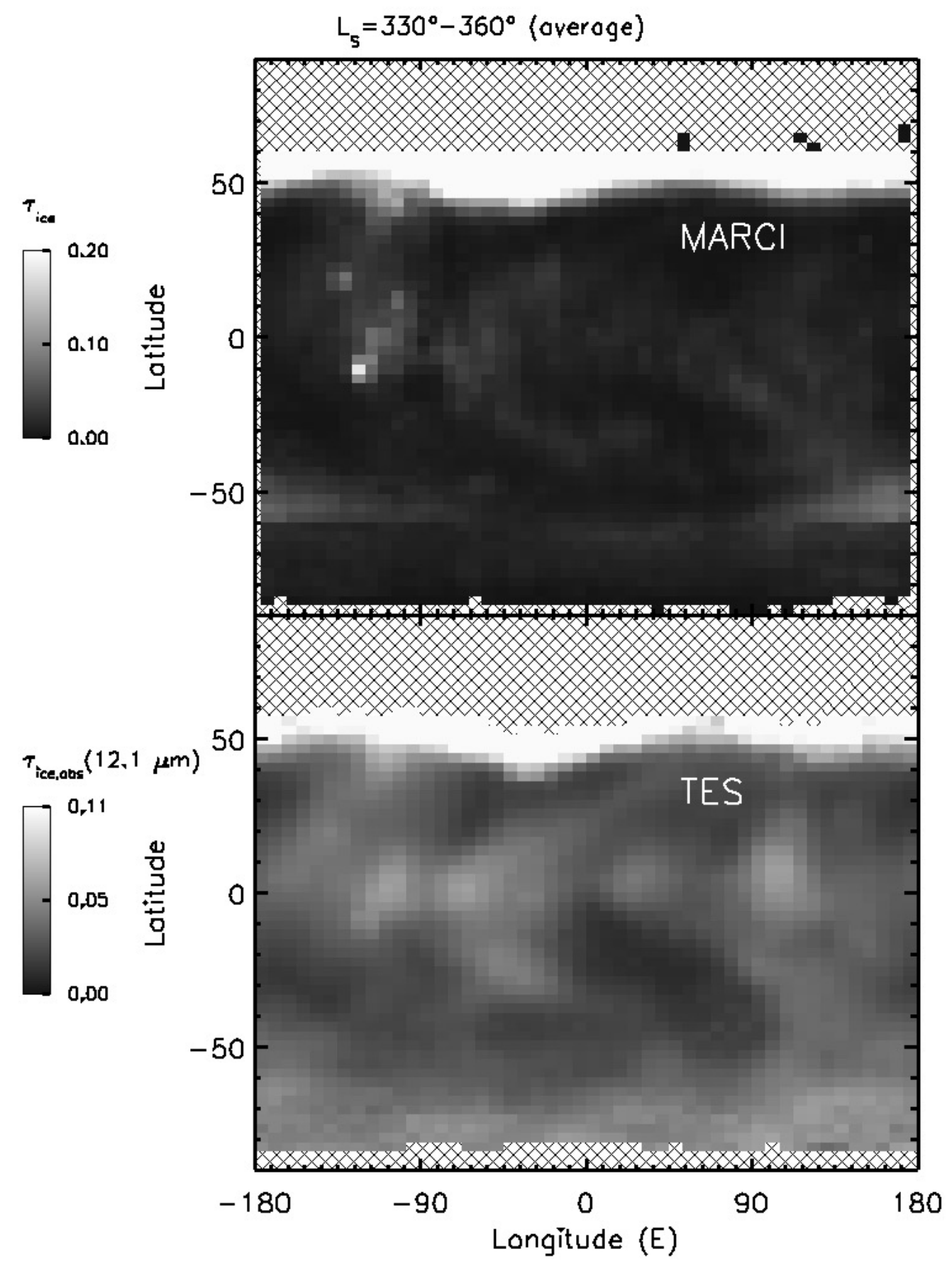

Figure 27: Same as Figure 16, but for $L_{S}=330^{\circ}-0^{\circ}$. The optical depth ranges have been decreased to allow for lower optical depth associated with the southern summer season. 
absorption $\tau_{\text {ice }}$ as LMD.

Figure 28 compares constant latitude cross sections (slices) through the MARCI and TES zonal means (shown in Figure 14) with the equivalent samples from the Ames and LMD model runs. The two Ames optical depth products are meant to be analogous to the MARCI and TES retrieval products, namely a UV extinction optical depth and an infrared absorption optical depth. The ratio of these two values is sensitive to the water ice particle sizes in the GCM. The MARCI and TES data are binned in latitude to $5^{\circ}$ to better compare with the GCM results, i.e., the Ames model grid is $6^{\circ} \times 5^{\circ}$ (longitude $\times$ latitude). Since we are using the high resolution output from Pottier et al. $(2017)\left(1^{\circ} \times 1^{\circ}\right)$, the LMD zonal $\tau_{i c e}$ are also binned to $5^{\circ}$ in latitude. The zonal means for both models are constructed from averages over the local time range 14h00-16h00.

The Ames and LMD MGCMs generally capture the observed water ice zonal mean behaviors of the ACB and the polar hoods. The amplitudes of the model $\tau_{i c e}$ for the $\mathrm{ACB}$, particularly at the equator and northward, are generally consistent with those of TES and MARCI. The models predict the seasonal behavior of the polar hood clouds, particularly the decay phases of the polar hoods.

Although the models reasonably predict the major features of the observed water ice cloud populations, discrepancies between the observations and the models exist. The models tend to produce an ACB that extends too far south, as can be seen from the model $\tau_{i c e}$ in the visible and IR in the panels for $10^{\circ}$ and $30^{\circ} \mathrm{S}$. Additionally, both models predict low latitude clouds that are thick compared to observations during the perihelion season. 

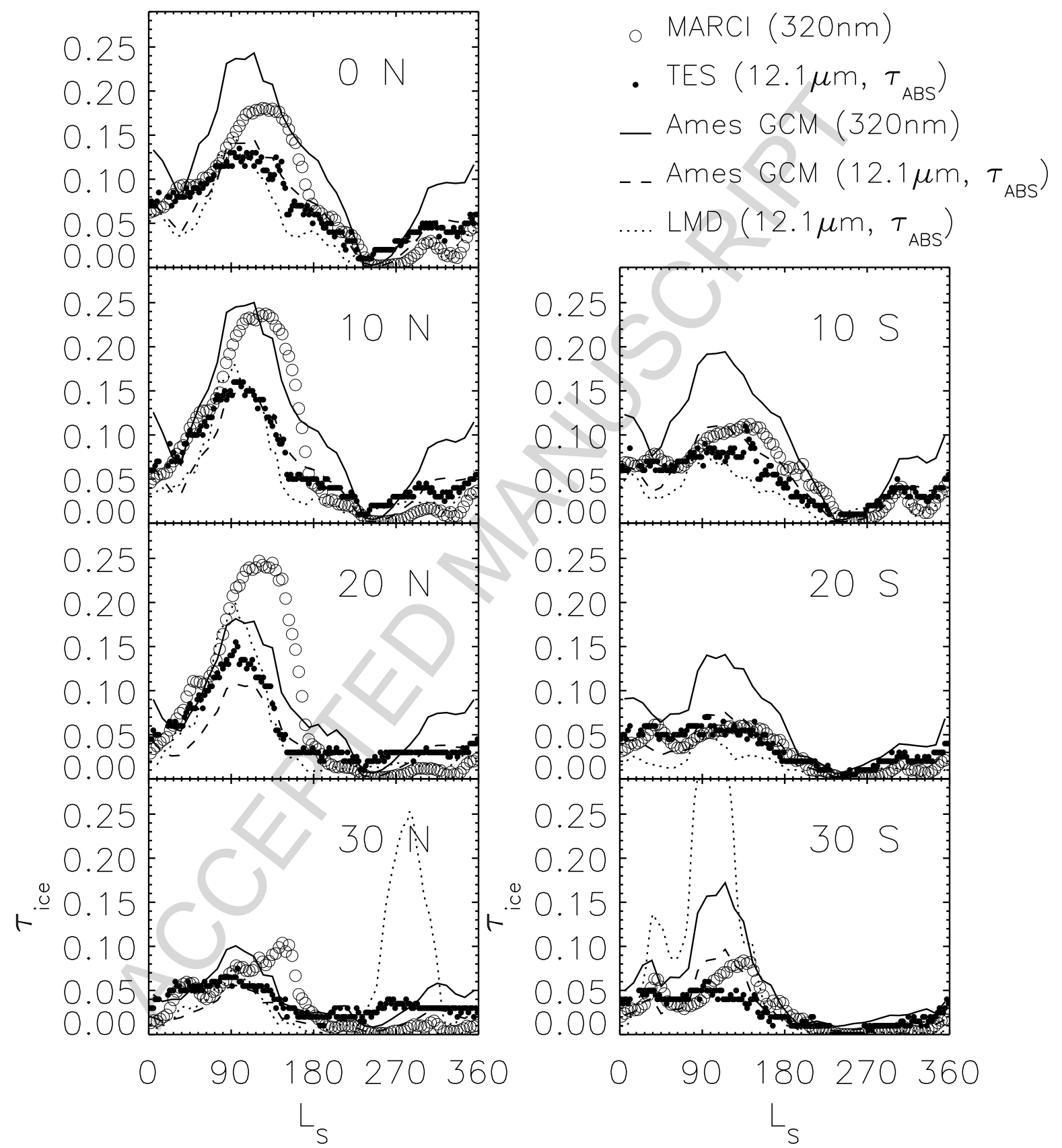

.... LMD $\left(12.1 \mu \mathrm{m}, \tau_{\text {ABS }}\right)$

0.25

0.20

0.15

0.10

0.05

0.00

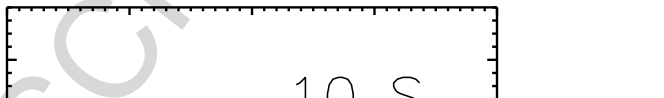

0.25

0.20

0.15

0.10

0.05

0.00

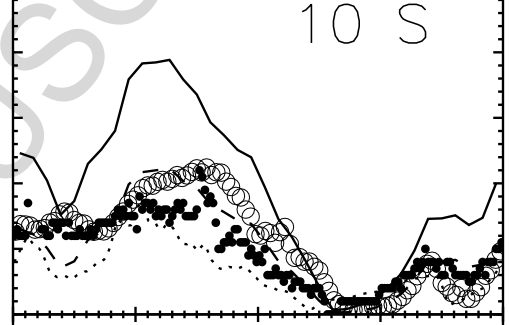

$\stackrel{0.0}{0}$

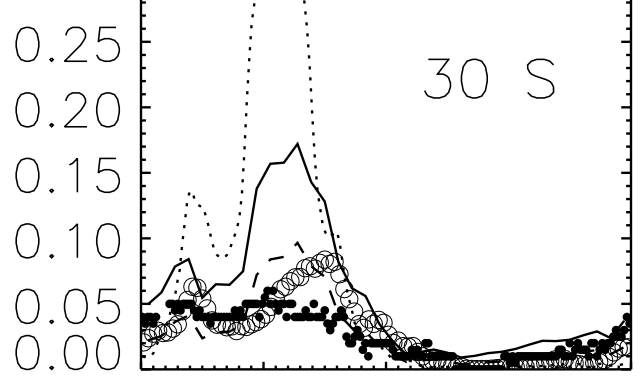

$0 \quad 90180270360$

Ls

Figure 28: Latitude cross sections of MARTE and TES zonal means (e.g., Figure 14 are compared to those from zonal means of the LMD and the Ames models. The particular latitudes are chosen to capture the ACB and the edges of the polar hood structure. The Ames optical depths are extinction in the UV and absorption in the IR. See text. 
Finally, both models predict polar hood clouds that are significantly too thick and extend too far equator-ward (particularly in the north) compared to MARCI and TES observations.

The previously discussed MARCI ACB asymmetric structure (with respect to $\mathrm{L}_{S}=90^{\circ}$ ) is quite evident in the $0^{\circ}$ and $10^{\circ} \mathrm{N}$ panels, as is the symmetric aspect of the TES $\tau_{i c e}$. The phasing (centroid) of the ACB in both models is much more consistent with that of TES than with MARCI. The predicted ratio between the UV and IR $\tau_{i c e}$ in the ACB from the Ames model remains nearly constant throughout the aphelion season, which indicates that the model does not predict significant particle size evolution. This remains an open area for future investigation.

For our comparisons to meridional behavior, we restrict the observations to those from MARCI. However, the lack of a corresponding UV $\tau_{i c e}$ in the LMD data set leads us to use water ice mass $\left(\mathrm{kg} / \mathrm{m}^{2}\right)$ as a proxy. We set the range on the color bars such that a specific grey level in the ice mass panels is equivalent to the MARCI optical depth associated with that color assuming a specific water ice size distribution. Specifically, one converts the mass to a column optical depth assuming spherical particles with an $r_{\text {eff }}=3 \mu \mathrm{m}$ (for which $Q_{\text {exet }}=1.6$ at $320 \mathrm{~nm}$ ). We consider both the high resolution and low resolution runs for the LMD GCM, $\left(1^{\circ} \times 1^{\circ}\right)$ and $\left(6^{\circ} \times 4^{\circ}\right)$, respectively. As with the zonal results, the Ames fields are averaged over the local time range 14h-16h. For the LMD results, the mass field is interpolated to exactly $15 \mathrm{~h}$ for each Mars day and longitude bin.

Figure 29 compares the MARCI $\tau_{\text {ice }}$ values with the GCM water ice column masses averaged over $\mathrm{L}_{S}=0^{\circ}-30^{\circ}$. The goal of this comparison is to 


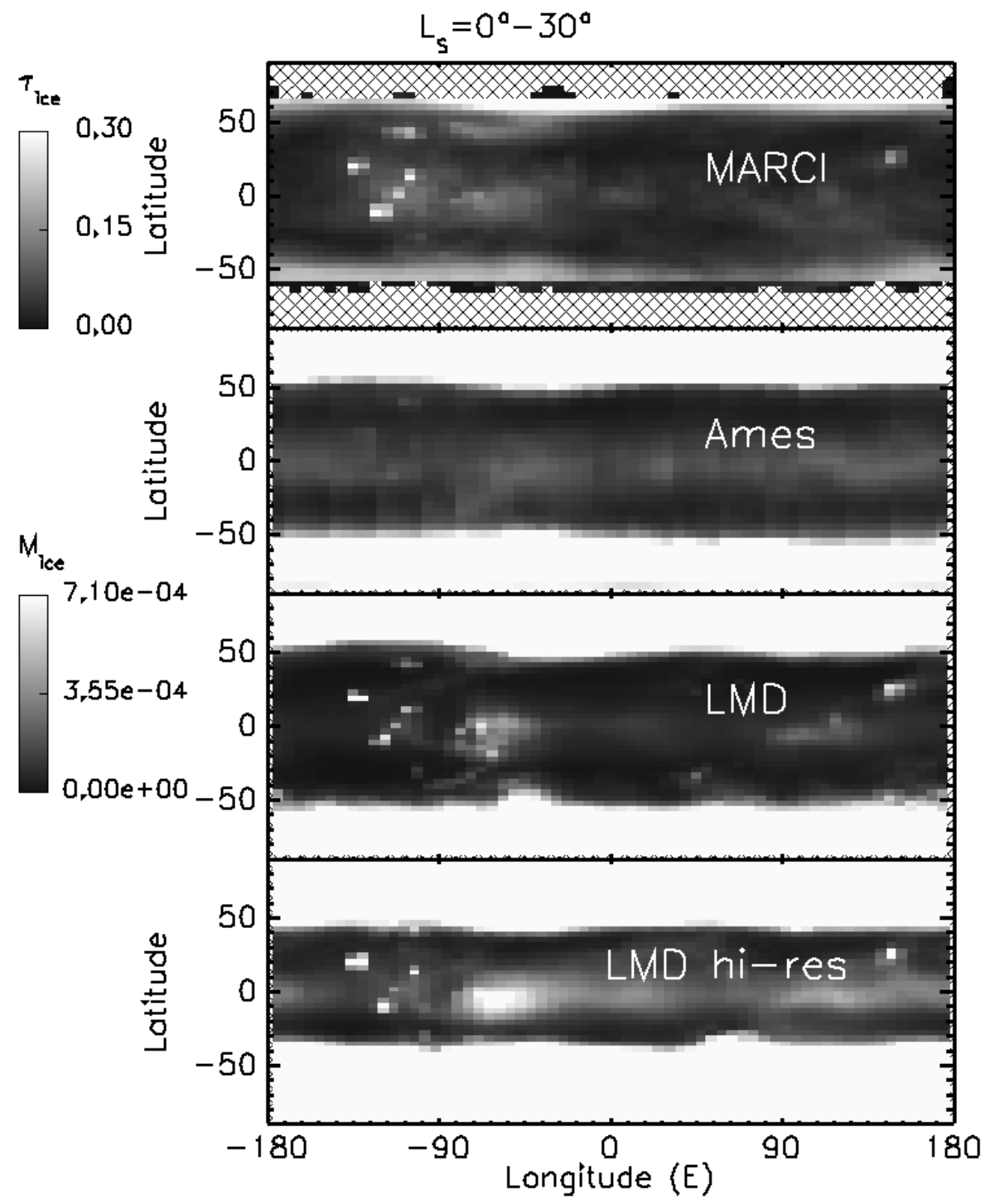

Figure 29: Comparison for the period of $L_{S}=0^{\circ}-30^{\circ}$ of MARCI water ice optical depth with the mass of water ice $\left(\mathrm{kg} / \mathrm{m}^{2}\right)$ predicted by the NASA-Ames and the LMD GCMs, where the latter is run at low- $\left(6^{\circ} \times 4^{\circ}\right)$ and high-resolution $\left(1^{\circ} \times 1^{\circ}\right)$. The high-resolution case is downsample to $6^{\circ} \times 4^{\circ}$ here for comparison. The range of the colorbars was chosen so that the same gray levels are equivalent water ice columns under the assumption of spherical particles with $r_{\text {eff }}=3 \mu \mathrm{m}\left(Q_{e x t}=77.6\right.$ for $\left.320 \mathrm{~nm}\right)$. See text. 
compare orographic-topographic clouds and the polar hoods. The difference between the two LMD models is the resolution. The higher resolution run output is binned to $6^{\circ} \times 4^{\circ}$ for comparison to MARCI and Ames. The LMD model does a good job with the orographic-topographic clouds, which are not seen in this period in the Ames model. The lack of predicted discrete orographic-topographic clouds by the Ames model likely reflects that model ingests a topography map that has been been more heavily smoothed that the one used in the LMD model. Both models are in good agreement with respect to the $\mathrm{PH}$ boundaries, though lower in latitude for the north $\mathrm{PH}$ as compared to MARCI. The primary difference between the two LMD cases is that the high resolution is generally much cloudier, particularly with the extent of the polar hoods and Valles Marineris clouds. Both models show the suggestion of a diffuse, low latitude band of clouds that is not clearly seen in MARCI (but seen in TES, see Figure 16). This feature is thicker for the LMD model high resolution case. In fact, it is a general trend for the high resolution model to produce more clouds than the lower resolution one; see also Figure 10 of Pottier et al. (2017).

We move to the ACB structure and the Hellas Basin with Figure 30, which shows the period $\mathrm{L}_{S}=120^{\circ}-150^{\circ}$. The focus is to compare orographictopographic clouds and the polar hoods. The Ames model reproduces undulations of more diffuse ACB structure, while resolving the cloud structures associated with Olympus Mons, Ascreus, Valles Marineris, Elysium Mons, and Hellas. As shown in Kahre et al. (2018), clouds in Hellas result from increased water transport just to the west of the basin and the regional dynamics of the basin itself. The LMD models add clouds associated with the 


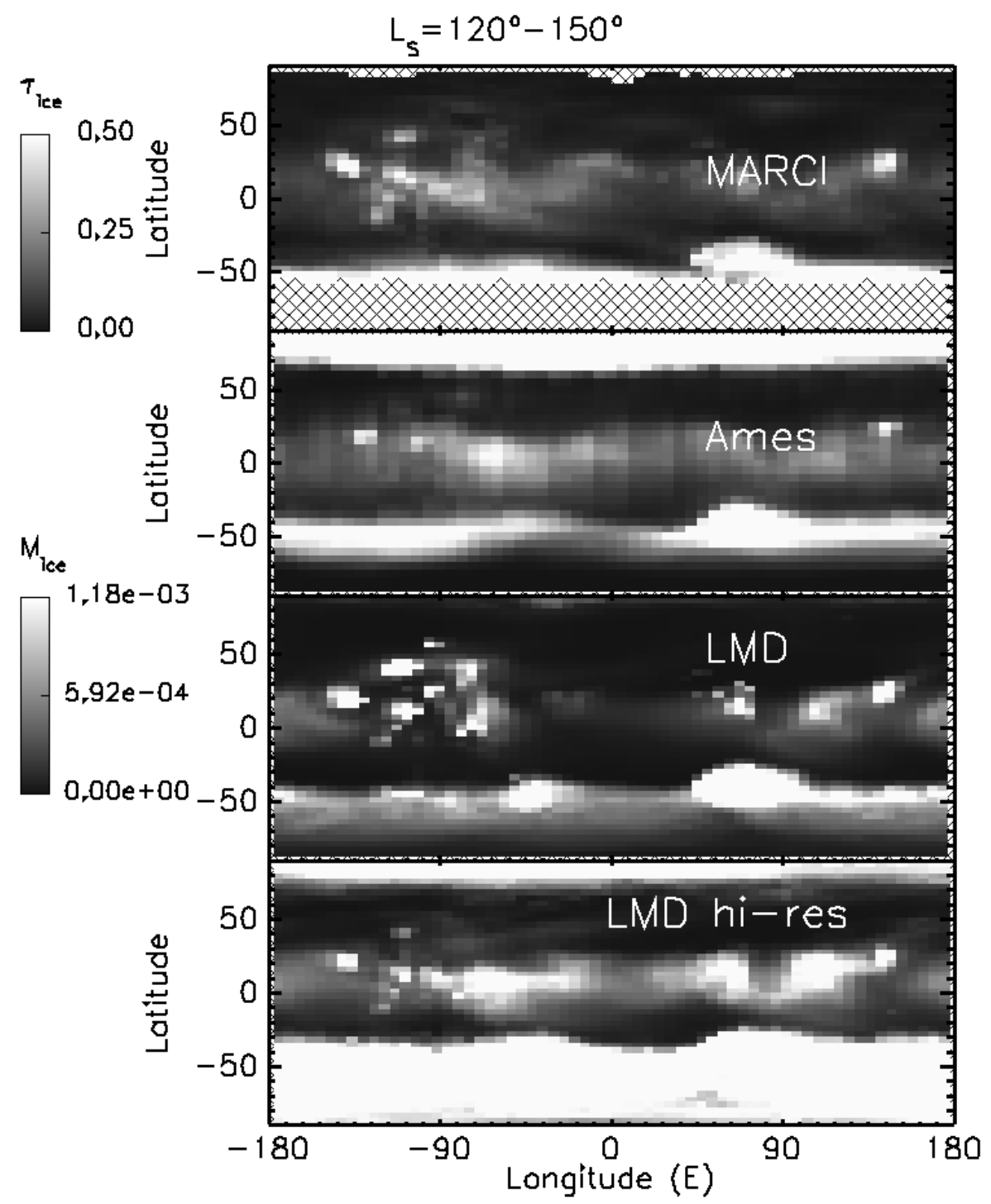

Figure 30: Comparison of MARCI water ice optical depth with the mass of water ice $\left(\mathrm{kg} / \mathrm{m}^{2}\right)$ predicted by the NASA-Ames and the LMD GCM (at low and high resolution) for the period $L_{S}=120^{\circ}-150^{\circ}$. See caption for Figure 29 . 
two more southern Tharsis volcanoes and Alba Mons. The LMD model is again cloudier in the high resolution run, with the lower resolution case missing some of the diffuse ACB structure. For both models, the correspondance of the polar hood structures is again good, including the feature associated with Argyre (near $50^{\circ} \mathrm{E}$ ). It is worth noting that both lower resolution simulations predict an annular south polar hood cloud feature during northern summer, whereas the higher resolution simulation produces clouds that extend to the south pole.

Lastly, we consider a final interesting case. Figure 31 covers the late southern summer through the equinox, $\mathrm{L}_{S}=330^{\circ}-360^{\circ}$. As with the first case, the LMD model reproduces the topographic structures seen in MARCI, including thicker clouds at Arsia Mons. Both models reproduce the boundaries of the northern $\mathrm{PH}$, including latitude of the perimeter. As discussed in Haberle et al. (2018b), the wave-2 structure in the equator-ward extent of the northern $\mathrm{PH}$ is produced by forced stationary waves. The models have clearly initiated the southern hood a bit too soon. The diffuse clouds observed at low latitudes in the models are generally absent in the MARCI data, though evident in TES, Figure 27. However, MARCI does find clouds associated with Valles Mariners (i.e., as seen in LMD), as well as with the "swooping" diffuse structure centered near Chyrse Planitia $\left(40^{\circ} \mathrm{E}, 10^{\circ}\right.$; both models)

\subsection{Public Data Products}

It is our goal to generate publicly available products similar to those shown in this paper, i.e., zonal products, latitude-longitude maps, and other summary products. We have begun this process, with curation and hosting 


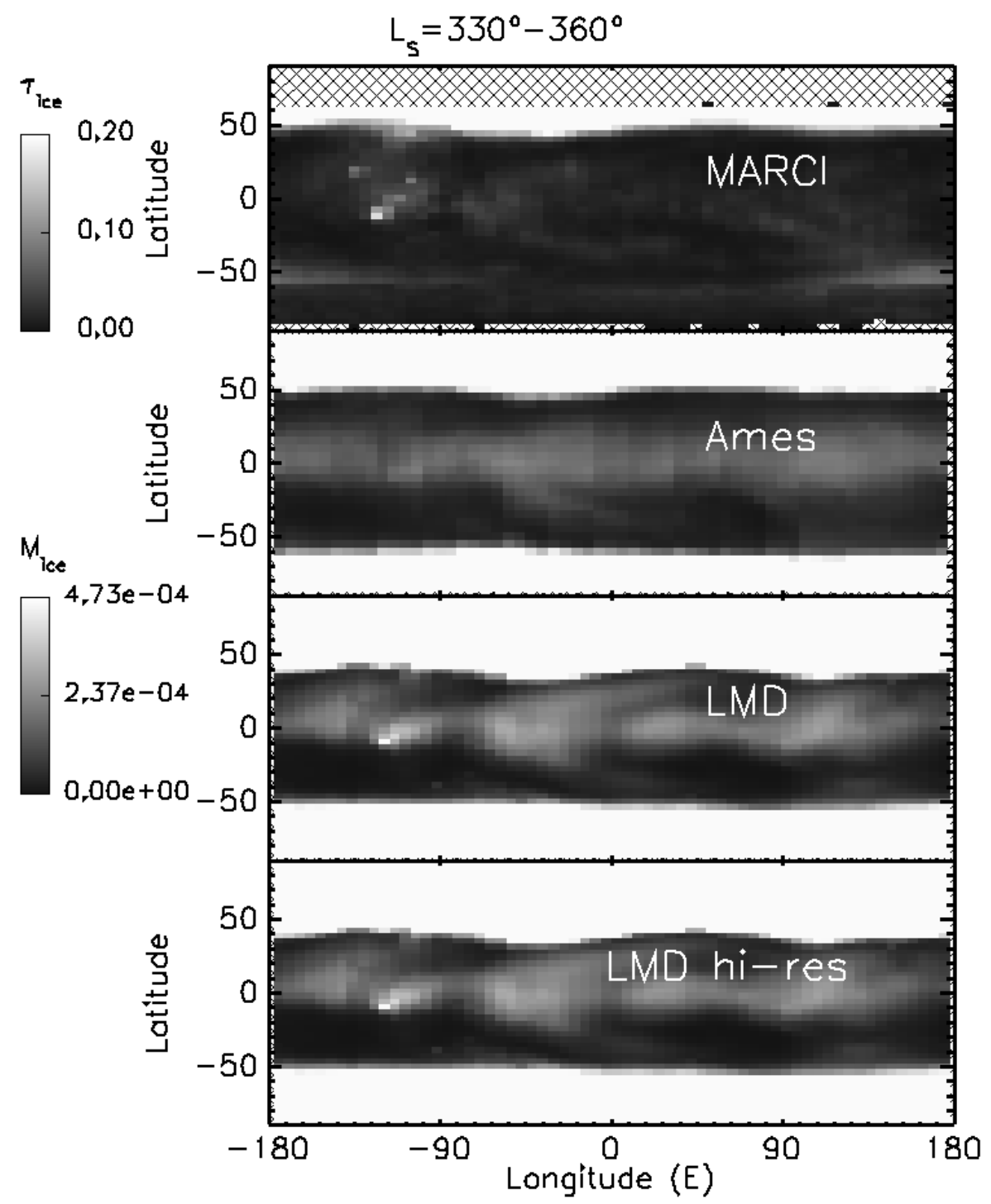

Figure 31: Comparison of MARCI water ice optical depth with the mass of water ice $\left(\mathrm{kg} / \mathrm{m}^{2}\right)$ predicted by the NASA-Ames and the LMD GCM (at low and high resolution) for the period $L_{S}=330^{\circ}-360^{\circ}$. See caption for Figure 29 . 
provided by Malin Space Science Systems: https://www.msss.com/mro_ marci_iceclouds/. Requests for specific products can be made by contacting the first author via e-mail.

\section{Summary}

With over 6 Mars Years of nearly continuous observations, the MARCI data set offers a unique opportunity to characterize water ice cloud distributions on scales from regional and global to mesoscale $(\sim 10 \mathrm{~km})$. The sun synchronous orbit and the time span of the observations allow for characterization of both seasonal and interannual changes. The ultraviolet Band 7 $(320 \mathrm{~nm})$ provides an enhanced sensitivity to water ice clouds with respect to the dark surface and the more neutral impact of dust aerosols (i.e., lower single-scattering albedo, similar to that of the surface). In order to take advantage of this extensive set of observations, we have developed an efficient, radiative-transfer based $\tau_{i c e}$ retrieval pipeline that process a full mapping day of retrievals on the order of 10 minutes (using 12 cores in 10 year-old machines). Along the way, we have derived:

1. an update to the camera (geometry) model parameters for the UV. The previous values in the NAIF/SPICE geometry files were not sufficient at even moderate emergence angles;

2. a zonal dust climatology based on CRISM Emission Phase Function retrievals. This database is available upon request from the first author, and will be archived on the same site where the water ice clouds products are being archived (https://www.msss.com/mro_marci_iceclouds/); 
3. a surface reflectance model for Band 7 with a spatially variable normalization coefficient (and extended to Band 6), including a coarse "albedo map" that illustrates the so-called UV reversal;

4. a water ice scattering phase function starting from discrete dipole approximation calculations for a droxtal shape, which is a representation of the polycrystalline habit used for terrestrial applications when the water ice particles are typically smaller than $5 \mu \mathrm{m}$.

The uncertainty in the $\tau_{i c e}$ retrievals is estimated from perturbation and monte carlo analysis to be $\sim 0.03$. However, this does not take into account $r_{\text {eff }}$ and phase function issues, which are very difficult without some additional constraints on the particle shape. While phase functions for irregular particles tend to be less sensitive to particle morphology than than those for analytical shapes (e.g., spheres), it remains a point of concern. This is particularly true when comparing observations with specific (and different) scattering angles, as opposed to looking at an average over a range of angles. Nevertheless, we have estimated that if Martian clouds have $r_{e f f}=1.5 \mu \mathrm{m}$, MARCI will overestimate $\tau_{i c e}$ by about $30 \%$. This is in contrast to have larger particles, e.g., 4-6 $\mu \mathrm{m}$, where the effect is typically less than a $10 \%$ underestimation of $\tau_{i c e}$.

From the start of mapping in November 2006 until the beginning of the global dust event in June 2018, the MARCI band 7 retrievals have resulted in over $220 \mathrm{~GB}$ of data products, most of which are the metadata associated with each pixel. Since this estimate includes storage as compressed, 2-byte integers, any tractable summary product will involve some degree of spatial and temporal binning. For use in this project, we implement an $8 \times 8$ pixel 
$\left(1^{\circ} \times 1^{\circ}\right)$ spatial summing scheme that reduces the data volume to approximately 2 GB. Using the resulting database, we performed several analyses that reveal several trends that merit mention:

1. Using a zonal representation, the seasonally Aphelion Cloud Belt (ACB) and polar hood $(\mathrm{PH})$ cloud boundaries demonstrate repeatable structure from year-to-year when averaged over the local time range 14h16h. The associated interannual variability of the ACB is relatively small (though detectable); larger at the boundaries of the PH. When viewed in blocks of approximately 30 minutes, there can be a significant diurnal variability, particularly in the interval 14h30-15h30. These local time effects also possess apreciable interanual variability.

2. Comparing annual (mean) zonal behavior with TES $\tau_{i c e}$ retrievals reveals a difference in the seasonal $\left(\mathrm{L}_{S}\right)$ variations of the ACB. The TES results have a variation that is effectively more symmetric in time (e.g., centered near $\mathrm{L}_{S}=90^{\circ}$, while the MARCI profiles are skewed or shifted to later in the northern summer $\left(\mathrm{L}_{S} \sim 120^{\circ}\right)$. There is nothing in the observed diurnal trends of the MARCI data that suggests a clear relationship to diurnal changes (i.e., 14 h00 of TES versus $15 \mathrm{~h} 00$ of MARCI). However, the THEMIS $\tau_{i c e}$ - a data set with observational characteristics much more similar to TES than to MARCI, but with a mean local time near $17 \mathrm{~h} 00$ - shows a shifted ACB profile as well. In addition, both SPICAM and OMEGA suggest ACB profiles similar to that observed by MARCI (and THEMIS).

3. Comparison of MARCI latitude-longitude maps with those from TES $\left(30^{\circ}\right.$ bins in $\left.\mathrm{L}_{S}\right)$ show a general consistency in $\tau_{i c e}$ for the ACB structure, 
orographic and topographic clouds associated with various volcanoes (including Elysium and Alba Mons) and Valles Marineris, and polar hood boundaries (where surface-atmosphere contrast is sufficient for a TES detection). Some discrepancies occur in terms of the relative amplitudes of the MARCI and TES $\tau_{i c e}$ values, which are anticipated to be a function of the additional smoothing inherent in the lontgitudinal binning of the TES data set and of particle size effects.

4. TES detected water ice clouds in Hellas at a season where there were no corresponding detections by MARCI or MOC, (Wang and Ingersoll, 2002). As this conflict occurs during the southern summer season, one may wish to consider the case where moderate amounts of dust obscure UV-visible wavelength detection of near-surface water ice clouds.

5. TES shows modest amounts of diffuse water ice opacity in late southern summer and early northern spring that have no counterpart in the MARCI data. Such clouds are predicted by the Ames and LMD MGCMs over this period.

6. MGCM zonal mean $\tau_{i c e}$ values for the ACB show reasonable agreement with the amplitudes observed by MARCI, though in zonal representation, the $\mathrm{L}_{S}$-profile of the models is more consistent with that of TES rather than MARCI, i.e., profiles are centered on $\mathrm{L}_{S}=90^{\circ}$. Both the Ames and LMD GCM are successful in predicting the observed decay phase of the hoods.

7. Comparing GCM water ice mass to MARCI optical depths, the LMD model reproduces the general behavior of the ACB structure and orographic/topographic clouds. The degree of correspondance in the cloudi- 
ness between MARCI and LMD is related to the model resolution. The high resolution grid generally produces more clouds, though this elevated cloudiness produces a better agreement with MARCI for the case of diffuse ACB structure. In comparison, the Ames model reproduces only part of the topographically-linked cloud structures but matches the more diffuse structure of the ACB. Both models match with the phasing (wave structure) of the north and south PH clouds.

8. Both models predict more diffuse structure at low latitudes for late southern summer and early northern spring than is seen in MARCI retrieved cloud optical depths. However, these predictions, particularly by the LMD model, are in better agreement with the TES observations.

Finally, it is the intention of the authors to provide $\tau_{i c e}$ products to the community. We have begun to distribute files at https://www.msss.com/ mro_marci_iceclouds/. While we hope to migrate to a more permanent platform, this remains a viable staring point for MARCI water ice data (as well as emailing the first author).

\section{Acknowledgments}

The authors thank the operations and support staff at Malin Space Science Systems for keeping the MARCI observations and data products flowing so well over the years, as well as hosting the water ice cloud products for public dissemination! This work was supported by NASA through JPL Contract 1275776. We thank the two anonymous referees for their very careful and thorough reading of the manuscript and the many improvements that they recommended. We also thank Michael Smith - for the TES gridded data 
products and many helpful discussions - and André Szantai for the current status of the OMEGA water ice clouds analysis.

\section{Appendix A. Surface Reflectance Map}

Characterizing the spatial variation in the reflectance of the surface can be accomplished using a simple modification of the cloud retrieval algorithm. Specifically, by choosing observations where the CRISM dust database provides reasonable coverage and the assumption of no water ice cloud opacity is appropriate (or where cloudy pixels can be easily excluded), the same LUTs may be employed to relate the observed radiance to a value of the Hapke w parameter (see $\S 3.4)$.

We chose three distinct observational epochs to optimize the solar illumination across the latitude range and to minimize the presence of water ice clouds. In addition, we require that the sample is sufficiently large to allow for filtering on photometric angles and to provide enough pixels in each spatial bin for robust statistical moments. The general description of these periods is given in Table A.5. The first two data sets are for seasons where clouds are generally not present. This was not possible for the epoch chosen to cover the northern middle and high latitudes, though we minimize the contribution of the aphelion cloud belt feature.

The generation of the reflectance map starts with the individual strips of the Hapke w parameter generated from the daily observations. Each of the strips is projected onto a cylindrical grid with a scale of 8 pixels per degree. A mean map for each epoch is then constructed with a resolution of 1 pixel per degree by combining all the individual strips in an arithmetic mean (700-800 
Table A.5: MARCI Band 7 Observations for Reflectance Map

\begin{tabular}{cccc}
\hline DOY Range & Year & L $_{S}$ Range & Latitude Range \\
\hline $150-210$ & 2009 & $275^{\circ}-312^{\circ}$ & $90^{\circ} \mathrm{S}-25 \mathrm{~N}$ \\
$317-366$ & 2010 & $180^{\circ}-208^{\circ}$ & $25^{\circ} \mathrm{S}-25^{\circ} \mathrm{N}$ \\
$65-115$ & 2010 & $60^{\circ}-82^{\circ}$ & $20^{\circ} \mathrm{N}-90^{\circ} \mathrm{N}$ \\
\hline
\end{tabular}

a Day of Year

a Range of latitudes used to populate the "mean map".

image strips). In order to minimize the errors associated with the departures from the idealized atmospheric state (i.e., presence of thin clouds, discrete dust clouds, etc.) and the effects of backscattering, we exclude pixels that:

1. have incidence angles greater than $70^{\circ}$, emergence angles greater than $5^{\circ}$, and a phase angles less than $20^{\circ}$;

2. have $\mathrm{w}$ values less than 0.03 or greater than 0.12 ; and

3. are within $\sim 5^{\circ}$ of the three Tharsis volcanoes, Olympus Mons, Alba Mons, and Elysium Mons.

These parameter ranges were set through numerical experimentation designed to minimize the variance of the pixel values in each $1^{\circ}$ bin. Then each of the mean maps from the three epochs is combined into a single map, resulting in Figure A.32.

Even with the selection criteria above, Figure A.32 retains noticeable artifacts associated with the atmospheric correction process and the relatively modest contribution of the surface to the observed radiance. These are removed by applying a $5 \times 5$ median filter. The "empty" or undefined pixels 
(typically caused by persistent surface ice (polar regions) and ice clouds (volcano and scattered low-latitude regions) are populated by taking pixels from a more highly smoothed $(11 \times 11$ boxcar $)$ version of Figure A.32.

Finally, we take into account the bias in the retrieved w values that results from the increased relative contribution of the atmosphere to the darker surface elements. This effect tends to produce an overcorrection (i.e., artificially lower $\mathrm{w}$ value). This adjustment is accomplished through a linear transformation of the range defined by the minimum and mean values in the derived $\mathrm{w}$ value distribution, [0.030,0.078], to one with a higher minimum value. We have adopted the range [0.05, 0.078]. Though somewhat ad hoc, it represents the minimum change required to remove obvious artifacts in cloud maps over the darkest surface features. The final product is that shown in Figures 6 and 7 .

\section{Appendix B. Water Ice Single Scattering Phase Function}

Having specified both the composition and the particle size, one needs to choose the particle shape. The motivation for the droxtal shape can be intuitively understood from the description provided by Ohtake (1970). In essence, the particle growth from supercooled water drops occurs so quickly that equilibrium is not reached. As a result, the particles form with incom-

plete hexagonal and rectangular faces. Such a complicated shape does not lend itself to exact numerical techniques for the calculation of the radiative properties. One must resort to a finite element approach. For example, Yang et al. (2003) use the Finite Different Time Domain algorithm. However, due to the wavelength and particle sizes of interest, we chose a different tech- 

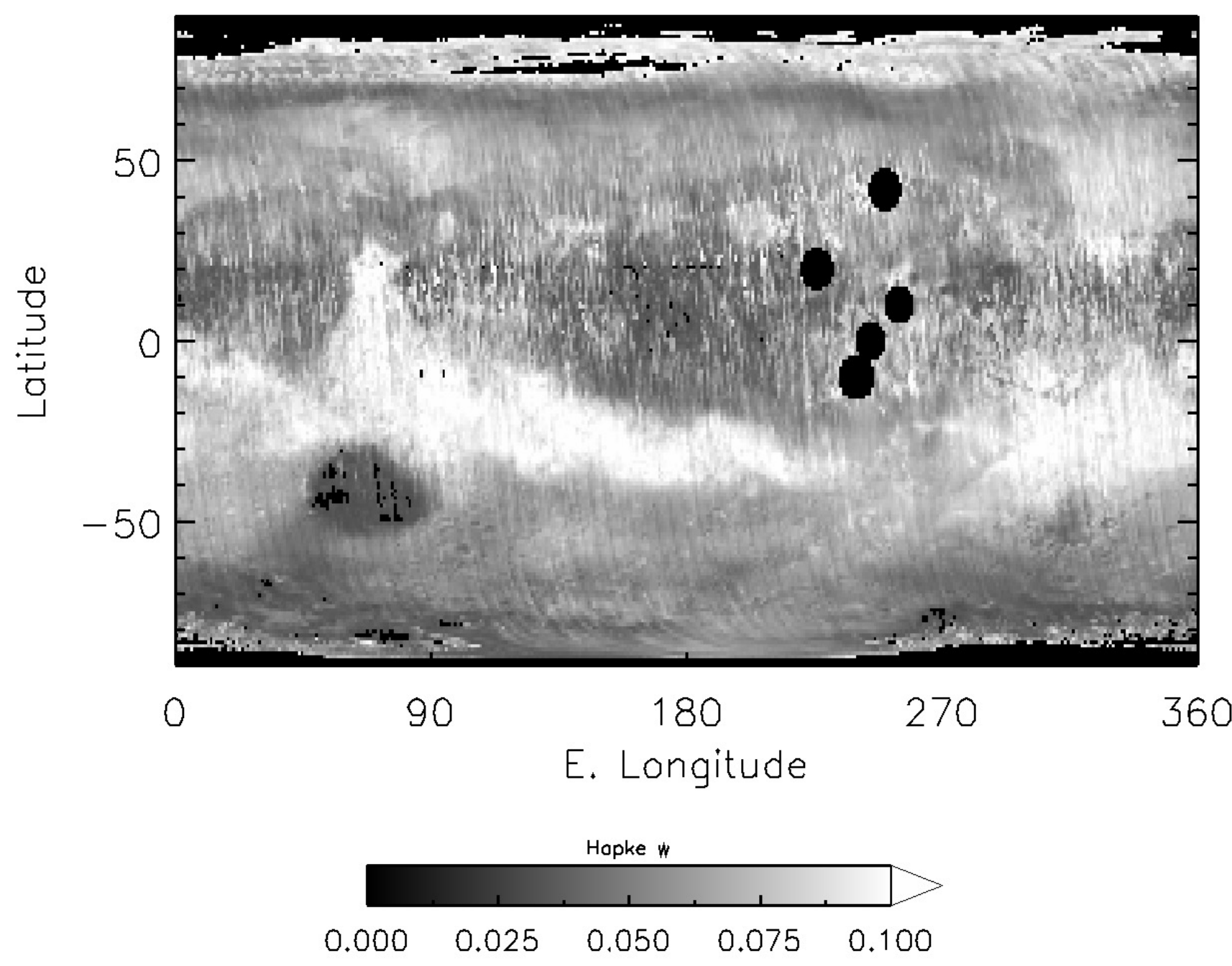

Figure A.32: Mean Map of Band 7 Hapke $w$ values derived from the observations in Table A.5, using the process described in the text. 
nique, namely the so-called the discrete dipole approximation (DDA, e.g., Draine and Flatau, 1994). Specifically, we employ an open-source version of the DDA, called DDSCAT, which is actively maintained and distributed via http://ddscat.wikidot.com. For the calculations presented here, we used version 7.2.1.

We generate a droxtal shape by starting with DDA sphere and then keep only the portion of the particle defined by the geometric prescription of Yang et al. (2003) with $\theta_{1}, \theta_{2}=30^{\circ}$ and $70^{\circ}$, and $\mathrm{R}=60$ (dipoles). Because the effective size parameter (i.e., $2 \pi r_{e f f} / \lambda$ ) is $\sim 60$, the circumscribing sphere has more than 900,000 dipole and the resulting droxtal - shown in Figure B.33 more than 720,000 dipoles.

Even with the large number of dipoles in the target, a single particle with $\mathrm{r}=3 \mu \mathrm{m}$ at a wavelength of $0.32 \mu \mathrm{m}$ is significantly stretching the DDA validity criteria (e.g., Draine and Flatau, 1994). Thus, instead of a size distribution with a range of particles sizes to produce the desired $r_{e f f}$ (and $v_{e f f}$, which would completely "break" the validity criteria, we use a single particle realization for an equivalent (sphere) radius of $3 \mu \mathrm{m}$. Fortunately, the many facets of the droxtal produce a much more subdued interference signature compared to more rounded shape like sphere, as can be seen in Figure B.34. However, by replacing the forward diffraction lobe of the DDA droxtal with that of the sphere calculations, say for scattering angles below $40^{\circ}$, one removes artifacts associated with the lack of a size integral.

The oscillations in the DDA droxtal phase function between $90^{\circ}$ and $180^{\circ}$ might not seem significant (e.g., Figure B.34) but, they do manifest themselves as arcs and stripes in the water ice optical depth maps. The effects can 
be mitigated by applying a smoothing function. However, a stronger artifact (a dark streak) remains after smoothing, occurring for phase angles between $80^{\circ}$ and $100^{\circ}$. In effect, making the droxtal phase function is "too bright" in this region. By replacing this feature with a linear function between the two angles, the phase function appears similar to that derived by Clancy et al. (2003). Consequently, our final phase function combines the shape of Clancy et al. (for scattering angles between $80^{\circ}-100^{\circ}$ ) with the smoothed DDA droxtal and the spherical particle diffraction lobe. The apparent offset between our adopted phase function and the original DDA phase function is due to the normalized nature of the scattering phase function, i.e.., the amplitude of the oscillations in near-forward scattering direction is artificially increased.

\section{Appendix C. Details of Uncertainty Analysis}

The uncertainty estimates in the first 3 lines of Table 3 are determined by perturbing the input parameter values used for the original retrievals in the example mapping day (10-April-2010, $\left.\mathrm{L}_{S}=75^{\circ}\right)$. This day includes a moderately large range of water ice optical depths $\left(0 \leq \tau_{i c e} \leq 0.8\right)$. A new set of retrievals is performed for each parameter perturbation, allowing for the calculation of the change in $\tau_{i c e}$ with respect to the relevant variable. Histograms of the errors (i.e., differences) are adequately represented by Gaussian distributions, and thus we adopt that functional form. Table 3 lists the mean and standard deviation of the histograms, where the latter is driven by the variability of $\tau_{i c e}$ within each observation. The desire to characterize the errors and their variability as a function $\tau_{i c e}$ motivates the use of full mapping day of observations with a range of $\tau_{\text {ice }}$ values. 


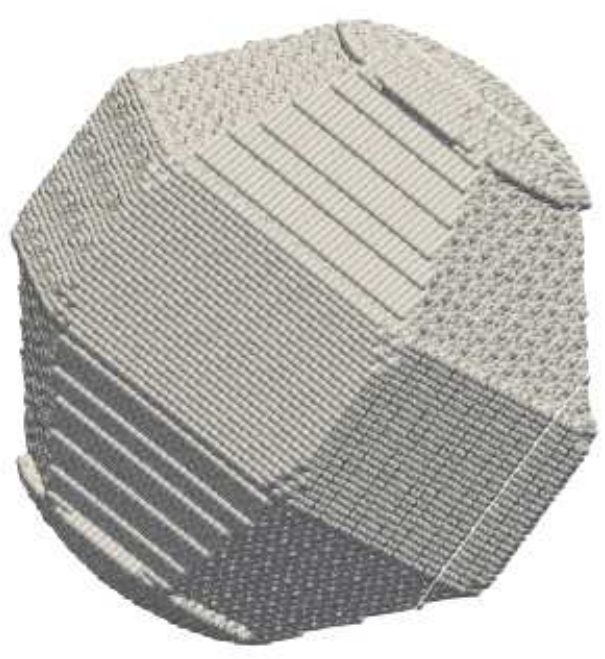

Figure B.33: Discrete Dipole Approximation realization of a droxtal from the geometric description of Yang et al. (2003). Starting with a circumscribing sphere of 904690 dipoles (via the CALLTARGET utility of DDSCAT), the resulting DDA target has 721,192 dipoles. The rounded caps are an artifact of our particle generation scheme; a true droxtal would have flat surfaces in these locations. The figure is rendered using Paraview (https : //www.paraview.org) software and the VTRCONVERT utility distributed with DDSCAT. 


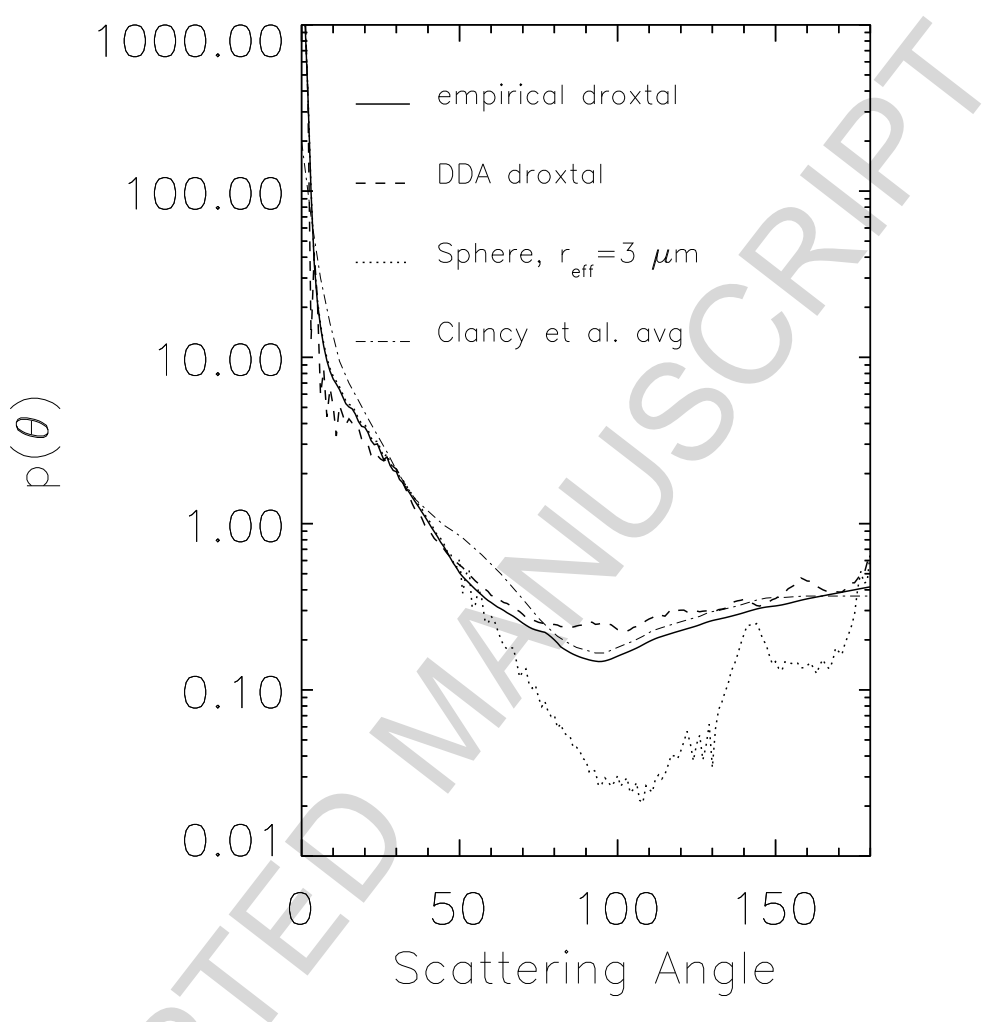

Figure B.34: Scattering phase functions for the DDA droxtal and equivalent sphere (dashed and dotted lines, respectively). These constituent functions are compared to the adopted phase function (solid line), which itself includes some additional empirical tuning. Specifically, the diffraction lobe from the sphere model and the functional dependence between $80^{\circ}$ and $100^{\circ}$ of the Clancy et al. (2003) average ice phase function (dot-dash curve) are combined with the smoothed DDA droxtal results. See text. 
In order to include potential effects of both spatial variability and a dependence on the amount of water ice $\left(\tau_{i c e}\right)$, we implement a monte carlo approach that builds on the previous perturbation analysis. Specifically, we take the following steps:

1. The errors for an individual retrieval (i.e., pixel) are simulated by selecting a $\tau_{i c e}$ and an error value for each of the three model parameters. $\tau_{i c e}$ is chosen from a uniform distribution between 0 an 1.0, which effectively represents the range in the example data set (i.e., 10-April 10-2010). Then, the associated error for the input parameters $\left(\tau_{\text {dust }}, \mathrm{w}\right.$, surface pressure) is chosen from a Gaussian distribution with a mean of zero and a standard deviation represented by the parenthetical \pm value in the first column. In other words, the LUT input values for each pixel are perturbed by an error that is drawn from a Gaussian distribution whose width is defined by relevant variance. In this way, we allow for errors from an entire distribution rather than a single representative value.

2. For each set of $\tau_{i c e}$ and the three error terms, the total error is calculated assuming no correlation between the terms.

3. The above process is repeated for several runs of 100,000 individual (pixel) retrieval simulations. Each run is is starting with a unique random number seed.

The net result of this monte carlo experiment is listed on the fifth line of Table 3. The mean optical depth error of 0.019 is an average across the full range of $\tau_{i c e}$ values. However, the types of errors considered tend to be 
constant rather than multiplicative. As such, they would not be expected to display a strong dependence on $\tau_{i c e}$.

We next consider observational errors, which are dominated by the uncertainty in the radiometric calibration, $\sim 5-7 \%$ range. Most of this error should be considered systematic in that it cannot be reduced by co-addition. In terms of the actual random component, the shot noise is less than $1 \%$. Using an error of $7 \%$, We repeat the perturbation exercises employed for the model input parameters. The results are listed on line 6 of Table 3, which shows that the uncertainty associated with radiometric error is similar to that of the total input uncertainty. In this case, the larger standard deviation is driven the variance due to the strong effects of $\tau_{i c e}$ and path length (incidence, emergence angles) on the observed radiance.

Finally, we combine the model input and calibration error terms using the same quadrature and the monte carlo schemes discussed above. The larger monte carlo estimate underlines the impact of considering the distribution of errors rather than a single value. In the interest of taking a conservative approach, we adopt the larger of the two values, $\Delta \tau_{i c e}=0.03$. 


\section{References}

Albee, A.L., Palluconi, F.D., Arvidson, R.E., 1998. Mars Global Surveyor Mission: Overview and Status. Science 279, 1671. doi:10.1126/science. 279.5357 .1671 .

Anderson, E., Leovy, C., 1978. Mariner 9 television limb observations of dust and ice hazes on Mars. Journal of Atmospheric Sciences 35, 723-734. doi:10.1175/1520-0469(1978)035<0723:MTLOOD>2 . . . CO; 2.

Bailey, M.P., Hallett, J., 2009. A Comprehensive Habit Diagram for Atmospheric Ice Crystals: Confirmation from the Laboratory, AIRS II, and Other Field Studies. Journal of Atmospheric Sciences 66, 2888. doi:10.1175/2009JAS2883.1.

Baker, B.A., Lawson, R.P., 2006. In Situ Observations of the Microphysical Properties of Wave, Cirrus, and Anvil Clouds. Part I: Wave Clouds. Journal of Atmospheric Sciences 63, 3160-3185. doi:10.1175/JAS3802.1.

Bell, J.F., Ansty, T.M., 2007. High spectral resolution UV to near-IR observations of Mars using HST/STIS. Icarus 191, 581-602. doi:10.1016/j . icarus. 2007.05.019.

Bell, J.F., Wolff, M.J., Malin, M.C., Calvin, W.M., Cantor, B.A., Caplinger, M.A., Clancy, R.T., Edgett, K.S., Edwards, L.J., Fahle, J., Ghaemi, F., Haberle, R.M., Hale, A., James, P.B., Lee, S.W., McConnochie, T., Noe Dobrea, E., Ravine, M.A., Schaeffer, D., Supulver, K.D., Thomas, P.C., 2009. Mars Reconnaissance Orbiter Mars Color Imager (MARCI): Instru- 
ment description, calibration, and performance. Journal of Geophysical Research (Planets) 114, 8. doi:10.1029/2008JE003315.

Benson, J.L., Bonev, B.P., James, P.B., Shan, K.J., Cantor, B.A., Caplinger, M.A., 2003. The seasonal behavior of water ice clouds in the Tharsis and Valles Marineris regions of Mars: Mars Orbiter Camera Observations. Icarus 165, 34-52. doi:10.1016/S0019-1035(03)00175-1.

Benson, J.L., James, P.B., Cantor, B.A., Remigio, R., 2006. Interannual variability of water ice clouds over major martian volcanoes observed by MOC. Icarus 184, 365-371. doi:10.1016/j.icarus.2006.03.014.

Benson, J.L., Kass, D.M., Kleinböhl, A., 2011. Mars' north polar hood as observed by the Mars Climate Sounder. Journal of Geophysical Research (Planets) 116, 3008. doi:10.1029/2010JE003693.

Benson, J.L., Kass, D.M., Kleinböhl, A., McCleese, D.J., Schofield, J.T., Taylor, F.W., 2010. Mars' south polar hood as observed by the Mars Climate Sounder. Journal of Geophysical Research (Planets) 115, 12015. doi:10.1029/2009JE003554.

Bertaux, J.L., Korablev, O., Perrier, S., Quémerais, E., Montmessin, F., Leblanc, F., Lebonnois, S., Rannou, P., Lefèvre, F., Forget, F., Fedorova, A., Dimarellis, E., Reberac, A., Fonteyn, D., Chaufray, J.Y., Guibert, S., 2006. SPICAM on Mars Express: Observing modes and overview of UV spectrometer data and scientific results. Journal of Geophysical Research (Planets) 111, S90. doi:10.1029/2006JE002690. 
Bibring, J.P., Soufflot, A., Berthé, M., Langevin, Y., Gondet, B., Drossart, P., Bouyé, M., Combes, M., Puget, P., Semery, A., Bellucci, G., Formisano, V., Moroz, V., Kottsov, V., Bonello, G., Erard, S., Forni, O., Gendrin, A., Manaud, N., Poulet, F., Poulleau, G., Encrenaz, T., Fouchet, T., Melchiori, R., Altieri, F., Ignatiev, N., Titov, D., Zasova, L., Coradini, A., Capacionni, F., Cerroni, P., Fonti, S., Mangold, N., Pinet, P., Schmitt, B., Sotin, C., Hauber, E., Hoffmann, H., Jaumann, R., Keller, U., Arvidson, R., Mustard, J., Forget, F., 2004. OMEGA: Observatoire pour la Minéralogie, l'Eau, les Glaces et l'Activité, in: Wilson, A., Chicarro, A. (Eds.), Mars Express: the Scientific Payload, pp. 37-49.

Cantor, B.A., James, P.B., Calvin, W.M., 2010. MARCI and MOC observations of the atmosphere and surface cap in the north polar region of Mars. Icarus 208, 61-81. doi:10.1016/j.icarus.2010.01.032.

Christensen, P.R., Bandfield, J.L., Hamilton, V.E., Ruff, S.W., Kieffer, H.H., Titus, T.N., Malin, M.C., Morris, R.V., Lane, M.D., Clark, R.L., Jakosky, B.M., Mellon, M.T., Pearl, J.C., Conrath, B.J., Smith, M.D., Clancy, R.T., Kuzmin, R.O., Roush, T., Mehall, G.L., Gorelick, N., Bender, K., Murray, K., Dason, S., Greene, E., Silverman, S., Greenfield, M., 2001. Mars Global Surveyor Thermal Emission Spectrometer experiment: Investigation description and surface science results. Journal of Geophysical Research (Planets) 106, 23823-23872. doi:10.1029/2000JE001370.

Christensen, P.R., Jakosky, B.M., Kieffer, H.H., Malin, M.C., McSween, Jr., H.Y., Nealson, K., Mehall, G.L., Silverman, S.H., Ferry, S., Caplinger, M., Ravine, M., 2004. The Thermal Emission Imaging System (THEMIS) 
for the Mars 2001 Odyssey Mission. SSR 110, 85-130. doi:10.1023/B: SPAC.0000021008.16305.94.

Clancy, R.T., Grossman, A.W., Wolff, M.J., James, P.B., Rudy, D.J., Billawala, Y.N., Sandor, B.J., Lee, S.W., Muhleman, D.O., 1996. Water vapor saturation at low altitudes around Mars aphelion: A key to Mars climate? Icarus 122, 36-62. doi:10.1006/icar.1996.0108.

Clancy, R.T., Montmessin, F., Benson, J., Daerden, F., Colaprete, A., Wolff, M.J., 2017. Mars Clouds, in The Atmosphere and Climate of Mars. chapter 5. pp. 42-75. doi:10.1017/9781139060172.005.

Clancy, R.T., Nair, H., 1996. Annual (perihelion-aphelion) cycles in the photochemical behavior of the global Mars atmosphere. Journal of Geophysical Research 101, 12785-12790. doi:10.1029/96JE00836.

Clancy, R.T., Wolff, M.J., Christensen, P.R., 2003. Mars aerosol studies with the mgs tes emission phase function observations: Optical depths, particle sizes, and ice cloud types versus latitude and solar longitude. Journal of Geophysical Research (Planets) 108, 5098. DOI: 10.1029/2003JE002058.

Clancy, R.T., Wolff, M.J., Lefèvre, F., Cantor, B.A., Malin, M.C., Smith, M.D., 2016. Daily global mapping of Mars ozone column abundances with MARCI UV band imaging. Icarus 266, 112-133. doi:10.1016/j.icarus . 2015.11.016.

Conrath, B., Curran, R., Hanel, R., Kunde, V., Maguire, W., Pearl, J., Pirraglia, J., Welker, J., 1973. Atmospheric and surface properties of Mars 
obtained by infrared spectroscopy on Mariner 9. Science 78, 4267-4278. doi:10.1029/JB078i020p04267.

Curran, R.J., Conrath, B.J., Hanel, R.A., Kunde, V.G., Pearl, J.C., 1973. Mars: Mariner 9 Spectroscopic Evidence for $\mathrm{H}_{2} \mathrm{O}$ Ice Clouds. Science 182, 381-383. doi:10.1126/science.182.4110.381.

Draine, B.T., Flatau, P.J., 1994. Discrete-dipole approximation for scattering calculations. Journal of the Optical Society of America A 11, 1491-1499. doi:10.1364/JOSAA.11.001491.

Formisano, V., Angrilli, F., Arnold, G., Atreya, S., Bianchini, G., Biondi, D., Blanco, A., Blecka, M.I., Coradini, A., Colangeli, L., Ekonomov, A., Esposito, F., Fonti, S., Giuranna, M., Grassi, D., Gnedykh, V., Grigoriev, A., Hansen, G., Hirsh, H., Khatuntsev, I., Kiselev, A., Ignatiev, N., Jurewicz, A., Lellouch, E., Lopez Moreno, J., Marten, A., Mattana, A., Maturilli, A., Mencarelli, E., Michalska, M., Moroz, V., Moshkin, B., Nespoli, F., Nikolsky, Y., Orfei, R., Orleanski, P., Orofino, V., Palomba, E., Patsaev, D., Piccioni, G., Rataj, M., Rodrigo, R., Rodriguez, J., Rossi, M., Saggin, B., Titov, D., Zasova, L., 2005. The Planetary Fourier Spectrometer (PFS) onboard the European Mars Express mission. Planetary and Space Science 53,963-974. doi:10.1016/j.pss.2004.12.006.

French, R.G., Gierasch, P.J., Popp, B.D., Yerdon, R.J., 1981. Global patterns in cloud forms on Mars. Icarus 45, 468-493. doi:10.1016/0019-1035(81) 90047-6.

Garvin, J.B., Figueroa, O., Naderi, F.M., 2001. NASA's New Mars Explo- 
ration Program: The Trajectory of Knowledge. Astrobiology 1, 439-446. doi:10.1089/153110701753593847.

Haberle, R.M., Kahre, M.A., Hollingsworth, J.L., Montmessin, F., Wilson, R.J., Urata, R.A., Brecht, A.S., Wolff, M.J., Kling, A.M., Schaeffer, J.R., 2018a. Documentation of the NASA/Ames Mars Global Climate Model: Simulations of the Present Seasonal Water Cycle. Icarus, submitted August 29, 2018.

Haberle, R.M., Kahre, M.A., Hollingsworth, J.L., Wolff, M.J., Barnes, J.R., 2018b. MARCI Observations of a Wave-Two Feature in the Norther Polar Hood of Mars: Interpretation of with the Ames Mars Global Circulation Model. Icarus , submitted Nov. 2018.

Hansen, J.E., Travis, L.D., 1974. Light scattering in planetary atmospheres. Space Science Reviews 16, 527-610. doi:10.1007/BF00168069.

Hapke, B., 1993. Theory of reflectance and emittance spectroscopy. Topics in Remote Sensing, Cambridge, UK: Cambridge University Press, _c1993.

Hapke, B., 2012. Theory of reflectance and emittance spectroscopy, 2nd ed. Cambridge, UK: Cambridge University Press, —c2012.

Ityaksov, D., Linnartz, H., Ubachs, W., 2008. Deep-UV absorption and Rayleigh scattering of carbon dioxide. Chemical Physics Letters 462, 3134. doi:10.1016/j.cplett.2008.07.049.

James, P.B., Thomas, P.C., Wolff, M.J., Bonev, B.P., 2007. MOC observations of four Mars year variations in the south polar residual cap of Mars. Icarus 192, 318-326. doi:10.1016/j . icarus.2007.07.014. 
Johnson, J.R., Grundy, W.M., Lemmon, M.T., Bell, J.F., Johnson, M.J., Deen, R., Arvidson, R.E., Farrand, W.H., Guinness, E., Hayes, A.G., Herkenhoff, K.E., Seelos, F., Soderblom, J., Squyres, S., 2006b. Spectrophotometric properties of materials observed by Pancam on the Mars Exploration Rovers: 2. Opportunity. Journal of Geophysical Research (Planets) 111, S16. doi:10.1029/2006JE002762.

Johnson, J.R., Grundy, W.M., Lemmon, M.T., Bell, J.F., Johnson, M.J., Deen, R.G., Arvidson, R.E., Farrand, W.H., Guinness, E.A., Hayes, A.G., Herkenhoff, K.E., Seelos, F., Soderblom, J., Squyres, S., 2006a. Spectrophotometric properties of materials observed by Pancam on the Mars Exploration Rovers: 1. Spirit. Journal of Geophysical Research (Planets) 111, S14. doi:10.1029/2005JE002494.

Kahn, R., 1984. The spatial and seasonal distribution of Martian clouds and some meteorological implications. Journal of Geophysical Research 89, 6671-6688. doi:10.1029/JA089iA08p06671.

Kahre, M.A., Haberle, R.M., Hollingsworth, J.L., Wolff, M.J., 2018. MARCIObserved Clouds in the Hellas Basin During NH Summer. Icarus, submitted Nov. 2018.

Langevin, Y., Bibring, J.P., Montmessin, F., Forget, F., Vincendon, M., Douté, S., Poulet, F., Gondet, B., 2007. Observations of the south seasonal cap of Mars during recession in 2004-2006 by the OMEGA visible/nearinfrared imaging spectrometer on board Mars Express. Journal of Geophysical Research (Planets) 112, E08S12. doi:10.1029/2006JE002841. 
Laszlo, I., Stamnes, K., Wiscombe, W.J., Tsay, S.C., 2010. Towards generalized boundary conditions in DISORT, in: 13th Conference on Atmospheric Radiation, p. P3.11. URL: https://ams.confex.com/ams/ 13CldPhy13AtRad/techprogram/paper_171283.htm.

Lefèvre, F., Bertaux, J.L., Clancy, R.T., Encrenaz, T., Fast, K., Forget, F., Lebonnois, S., Montmessin, F., Perrier, S., 2008. Heterogeneous chemistry in the atmosphere of Mars. Nature 454, 971-975. doi:10.1038/nature07116.

Leovy, C.B., Briggs, G.A., Smith, B.A., 1973. Mars Atmosphere during the Mariner 9 Extended Mission: Television Results. Journal of Geophysical Research 78, 4252-4266. doi:10.1029/JB078i020p04252.

Lin, Z., Stamnes, S., Jin, Z., Laszlo, I., Tsay, S.C., Wiscombe, W.J., Stamnes, K., 2015. Improved discrete ordinate solutions in the presence of an anisotropically reflecting lower boundary: Upgrades of the DISORT computational tool. Journal of Quantitative Spectroscopy and Radiative Transfer 157, 119-134. doi:10.1016/j . jqsrt.2015.02.014.

Madeleine, J.B., Forget, F., Millour, E., Navarro, T., Spiga, A., 2012a. The influence of radiatively active water ice clouds on the Martian climate. Geophysical Research Letters 39, 23202. doi:10.1029/2012GL053564.

Madeleine, J.B., Forget, F., Spiga, A., Wolff, M.J., Montmessin, F., Vincendon, M., Jouglet, D., Gondet, B., Bibring, J.P., Langevin, Y., Schmitt, B., 2012b. Aphelion water-ice cloud mapping and property retrieval us- 
ing the OMEGA imaging spectrometer onboard Mars Express. Journal of Geophysical Research (Planets) 117, 0. doi:10.1029/2011JE003940.

Malin, M.C., Calvin, W., Clancy, R.T., Haberle, R.M., James, P.B., Lee, S.W., Thomas, P.C., Caplinger, M.A., 2001. The mars color imager (marci) on the mars climate orbiter. Journal of Geophysical Research (Planets) 106, 17651-17672. doi:10.1029/1999JE001145.

Malin, M.C., Calvin, W.M., Cantor, B.A., Clancy, R.T., Haberle, R.M., James, P.B., Thomas, P.C., Wolff, M.J., Bell, J.F., Lee, S.W., 2008. Climate, weather, and north polar observations from the Mars Reconnaissance Orbiter Mars Color Imager. Icarus 194, 501-512. doi:10.1016/j.icarus. 2007.10 .016 .

Malin, M.C., Danielson, G.E., Ingersoll, A.P., Masursky, H., Veverka, J., Ravine, M.A., Soulanille, T.A., 1992. Mars Observer camera. Journal of Geophysical Research (Planets) 97, 7699-7718. doi:10.1029/92JE00340.

Malin Space Science Systems (MSSS), 2005. Mars Reconnaissance Orbiter, Mars Color Imager (MARCI), and Context Camera (CTX) pre-flight calibration report. Technical Report MSSS-CTX-DOC-2002. Malin space Science Systems. San Diego, CA. URL: http://marswatch . astro. cornell. edu/MARCI/Calibration_Report/MARCI_CTX_Cal_Report_v1.5.pdf.

Mateshvili, N., Fussen, D., Vanhellemont, F., Bingen, C., Dekemper, E., Loodts, N., Tetard, C., 2009. Water ice clouds in the Martian atmosphere: Two Martian years of SPICAM nadir UV measurements. Planetary and Space Science 57, 1022-1031. doi:10.1016/j ·pss. 2008.10.007. 
Mateshvili, N., Fussen, D., Vanhellemont, F., Bingen, C., Dodion, J., Montmessin, F., Perrier, S., Dimarellis, E., Bertaux, J.L., 2007. Martian ice cloud distribution obtained from SPICAM nadir UV measurements. Journal of Geophysical Research (Planets) 112, 7004. doi:10 . 1029/2006 JE002827.

McCleese, D.J., Schofield, J.T., Taylor, F.W., Calcutt, S.B., Foote, M.C., Kass, D.M., Leovy, C.B., Paige, D.A., Read, P.L., Zurek, R.W., 2007. Mars Climate Sounder: An investigation of thermal and water vapor structure, dust and condensate distributions in the atmosphere, and energy balance of the polar regions. Journal of Geophysical Research (Planets) 112, 5. doi:10.1029/2006JE002790.

Menon, P., Wagner, S., Demack, S., Graat, E., Lee, K., Schulze, W., 2017. 50,000 Laps Around Mars: Navigating the Mars Reconnaissance Orbiter Through the Extended Missions (January 2009 - March 2017), in: 28th International Symposium on Space Flight Dynamics, pp. ISTS-2017-d110/ISSFD-2017-110. 28th International Symposium on Space Flight Dynamics ; Conference date: 03-06-2017 Through 09-06-2012.

Michaels, T.I., Colaprete, A., Rafkin, S.C.R., 2006. Significant vertical water transport by mountain-induced circulations on Mars. GRL 33, L16201. doi:10.1029/2006GL026562.

Mishchenko, M.I., Travis, L.D., Lacis, A.A., 2002. Scattering, absorption, and emission of light by small particles. Cambridge, U.K. : Cambridge University Press, 2002. 
Montmessin, F., Forget, F., Rannou, P., Cabane, M., Haberle, R.M., 2004. Origin and role of water ice clouds in the Martian water cycle as inferred from a general circulation model. Journal of Geophysical Research (Planets) 109, 10004. doi:10.1029/2004JE002284.

Murchie, S., Arvidson, R., Bedini, P., Beisser, K., Bibring, J.P., Bishop, J., Boldt, J., Cavender, P., Choo, T., Clancy, R.T., Darlington, E.H., Des Marais, D., Espiritu, R., Fort, D., Green, R., Guinness, E., Hayes, J., Hash, C., Heffernan, K., Hemmler, J., Heyler, G., Humm, D., Hutcheson, J., Izenberg, N., Lee, R., Lees, J., Lohr, D., Malaret, E., Martin, T., McGovern, J.A., McGuire, P., Morris, R., Mustard, J., Pelkey, S., Rhodes, E., Robinson, M., Roush, T., Schaefer, E., Seagrave, G., Seelos, F., Silverglate, P., Slavney, S., Smith, M., Shyong, W.J., Strohbehn, K., Taylor, H., Thompson, P., Tossman, B., Wirzburger, M., Wolff, M., 2007. Compact reconnaissance imaging spectrometer for mars (crism) on mars reconnaissance orbiter (mro). Journal of Geophysical Research (Planets) 112. DOI: 10.1029/2006JE002682.

Navarro, T., Madeleine, J.B., Forget, F., Spiga, A., Millour, E., Montmessin, F., Määttänen, A., 2014. Global climate modeling of the Martian water cycle with improved microphysics and radiatively active water ice clouds. Journal of Geophysical Research (Planets) 119, 1479-1495. doi:10.1002/ 2013JE004550, arXiv:1310.1010.

Ohtake, T., 1970. Unusual Crystal in Ice Fog. Journal of Atmospheric Sciences 27, 509-513. doi:10.1175/1520-0469(1970) 027<0509:UCIIF> $2.0 . \mathrm{CO} ; 2$. 
Olsen, K.S., Forget, F., Madeleine, J.B., Szantai, A., Audouard, J., Geminale, A., Altieri, F., Bellucci, G., Oliva, F., Montabone, L., Wolff, M., 2019. Retrieval of the water ice column and physical properties of water-ice clouds in the Martian atmosphere using the OMEGA imaging spectrometer. Icarus, in pressdoi:https://doi.org/10.1016/j.icarus.2019.03. 006.

Pearl, J.C., Smith, M.D., Conrath, B.J., Bandfield, J.L., Christensen, P.R., 2001. Observations of Martian ice clouds by the Mars Global Surveyor Thermal Emission Spectrometer: The first Martian year. Journal of Geophysical Research 106, 12325-12338. doi:10.1029/1999JE001233.

Pottier, A., Forget, F., Montmessin, F., Navarro, T., Spiga, A., Millour, E., Szantai, A., Madeleine, J.B., 2017. Unraveling the martian water cycle with high-resolution global climate simulations. Icarus 291, 82-106. doi:10.1016/j.icarus.2017.02.016.

Richardson, M.I., Wilson, R.J., Rodin, A.V., 2002. Water ice clouds in the Martian atmosphere: General circulation model experiments with a simple cloud scheme. Journal of Geophysical Research (Planets) 107, 5064. doi:10.1029/2001JE001804.

Schmidt, R., 2003. Mars Express-ESA's first mission to planet Mars. Acta Astronautica 52, 197-202.

Semenov, B., 2007. MARCI Instrument Kernel. Technical Report version 10. NAIF/NASA Jet Propulsion Laboratory. Pasadena, CA. URL: http: //naif.jpl.nasa.gov/pub/naif/MRO/kernels/ik/mro_marci_v10.ti. 
Slipher, E.C., 1962. A photographic history of Mars, 1905-1961.

Smith, D.E., Zuber, M.T., Solomon, S.C., Phillips, R.J., Head, J.W., Garvin, J.B., Banerdt, W.B., Muhleman, D.O., Pettengill, G.H., Neumann, G.A., Lemoine, F.G., Abshire, J.B., Aharonson, O., Brown, C., D., Hauck, S.A., Ivanov, A.B., McGovern, P.J., Zwally, H.J., Duxbury, T.C., 1999. The Global Topography of Mars and Implications for Surface Evolution. Science 284, 1495. doi:10.1126/science.284.5419.1495.

Smith, M.D., 2004. Interannual variability in TES atmospheric observations of Mars during 1999-2003. Icarus 167, 148-165. doi:10.1016/ S0019-1035(03) 00287-2.

Smith, M.D., 2008. Spacecraft Observations of the Martian Atmosphere. Annual Review of Earth and Planetary Sciences 36, 191-219. doi:10. 1146/annurev . earth. 36.031207.124334.

Smith, M.D., 2009. THEMIS observations of Mars aerosol optical depth from 2002-2008. Icarus 202, 444-452. doi:10.1016/j . icarus .2009.03.027.

Smith, M.D., Wolff, M.J., Clancy, R.T., Kleinböhl, A., Murchie, S.L., 2013. Vertical distribution of dust and water ice aerosols from CRISM limbgeometry observations. Journal of Geophysical Research (Planets) 118, 321-334. doi:10.1002/jgre. 20047.

Sneep, M., Ubachs, W., 2005. Direct measurement of the Rayleigh scattering cross section in various gases. Journal of Quantitative Spectroscopy and Radiative Transfer 92, 293-310. doi:10.1016/j.jqsrt.2004.07.025. 
Stamnes, K., Tsay, S.C., Jayaweera, K., Wiscombe, W., 1988. Numerically stable algorithm for discrete-ordinate-method radiative transfer in multiple scattering and emitting layered media. Applied Optics 27, 2502-2509.

Szantai, A., Audouard, J., Forget, F., Madeleine, J.B., Pottier, A., Millour, E., Gondet, B., Langevin, Y., Bibring, J.P., 2017. Martian cloud coverage and diurnal cloud life cycle derived from gridded Mars/Express OMEGA data. European Planetary Science Congress 11, EPSC2017-879.

Szantai, A., Wolff, M., Audouard, J., Forget, F., Madeleine, J.B., Pottier, A., Millour, E., Gondet, B., Langevin, Y., Bibring, J.P., 2018. Construction and use of a 4D cloud database derived from MEx/OMEGA data - Cloud life cycle over polar regions. European Planetary Science Congress 12, EPSC2018-960.

Thomas, G.E., Stamnes, K., 2002. Radiative Transfer in the Atmosphere and Ocean. Radiative Transfer in the Atmosphere and Ocean, by Gary E. Thomas and Knut Stamnes. Cambridge Atmospheric and Space Science Series, Cambridge University Press, 2002, 546 pp.

Thomas, P., Veverka, J., 1986. Red/violet contrast reversal on Mars - Significance for eolian sediments. Icarus 66, 39-55. doi:10.1016/0019-1035(86) 90005-9.

Thuman, W.C., Robinson, E., 1954. Studies of Alaskan Ice-Fog Particles. Journal of Atmospheric Sciences 11, 151-156. doi:10.1175/ $1520-0469(1954) 011<0151:$ SOAIFP $>2$. $0 . \mathrm{CO} ; 2$. 
Wang, H., Fisher, J.A., 2009. North polar frontal clouds and dust storms on Mars during spring and summer. Icarus 204, 103-113. doi:10.1016/j . icarus.2009.05.028.

Wang, H., Ingersoll, A.P., 2002. Martian clouds observed by Mars Global Surveyor Mars Orbiter Camera. Journal of Geophysical Research (Planets) 107, 5078. doi:10.1029/2001JE001815.

Warren, S.G., Brandt, R.E., 2008. Optical constants of ice from the ultraviolet to the microwave: A revised compilation. Journal of Geophysical Research (Atmospheres) 113, D14220. doi:10.1029/2007JD009744.

Willame, Y., Vandaele, A.C., Depiesse, C., Lefèvre, F., Letocart, V., Gillotay, D., Montmessin, F., 2017. Retrieving cloud, dust and ozone abundances in the Martian atmosphere using SPICAM/UV nadir spectra. Planetary and Space Science 142, 9-25. doi:10.1016/j.pss . 2017.04.011.

Wilson, R.J., Lewis, S.R., Montabone, L., Smith, M.D., 2008. Influence of water ice clouds on Martian tropical atmospheric temperatures. Geophysical Research Letters 35, 7202. doi:10.1029/2007GL032405.

Wolff, M.J., Clancy, R.T., 2003. Constraints on the size of Martian aerosols from Thermal Emission Spectrometer observations. Journal of Geophysical Research (Planets) 108, 5097. doi:10.1029/2003JE002057.

Wolff, M.J., Lopéz-Valverde, M., Madeleine, J.B., Wilson, R.J., Smith, M.D., Fouchet, T., Delory, G.T., 2017. Radiative Process: Techniques and Applications in The Atmosphere and Climate of Mars. chapter 6. pp. 76-105. doi:10.1017/9781139060172.006. 
Wolff, M.J., Smith, M.D., Clancy, R.T., Arvidson, R., Kahre, M., Seelos, F., Murchie, S., Savijärvi, H., 2009. Wavelength dependence of dust aerosol single scattering albedo as observed by the Compact Reconnaissance Imaging Spectrometer. Journal of Geophysical Research (Planets) 114, 0. doi:10.1029/2009JE003350.

Wolff, M.J., Todd Clancy, R., Goguen, J.D., Malin, M.C., Cantor, B.A., 2010. Ultraviolet dust aerosol properties as observed by MARCI. Icarus 208, 143-155. doi:10.1016/j .icarus. 2010.01.010.

Yang, P., Baum, B.A., Heymsfield, A., Hu, Y.X., Huang, H.L., Tsau, S.C., Ackerman, S., 2003. Single scattering properties of droxtals. Journal of Quantitative Spectroscopy and Radiative Transfer 79, 1159. doi:10.1016/ S0022-4073(02)00347-3.

Zasova, L., Formisano, V., Moroz, V., Grassi, D., Ignatiev, N., Giuranna, M., Hansen, G., Blecka, M., Ekonomov, A., Lellouch, E., Fonti, S., Grigoriev, A., Hirsch, H., Khatuntsev, I., Mattana, A., Maturilli, A., Moshkin, B., Patsaev, D., Piccioni, G., Rataj, M., Saggin, B., 2005. Water clouds and dust aerosols observations with PFS MEX at Mars. Planetary and Space Science 53, 1065-1077. doi:10.1016/j.pss.2004.12.010.

Zurek, R.W., Smrekar, S.E., 2007. An overview of the Mars Reconnaissance Orbiter (MRO) science mission. Journal of Geophysical Research (Planets) 112, 5. doi:10.1029/2006JE002701. 


\section{Highlights}

- The zonal behavior is dominated by aphelion cloud belt (ACB) and polar hood structures.

- The MARCI ACB can demonstrate appreciable diurnal change and annual variability.

- MARCI ACB observations show differences with those of Thermal Emission Spectrometer.

- Dynamical models can capture the general behavior seen by the MARCI ACB and polar hoods. 
$L_{s}=115.6, M Y=29$ (21-August-2008) East Longitude

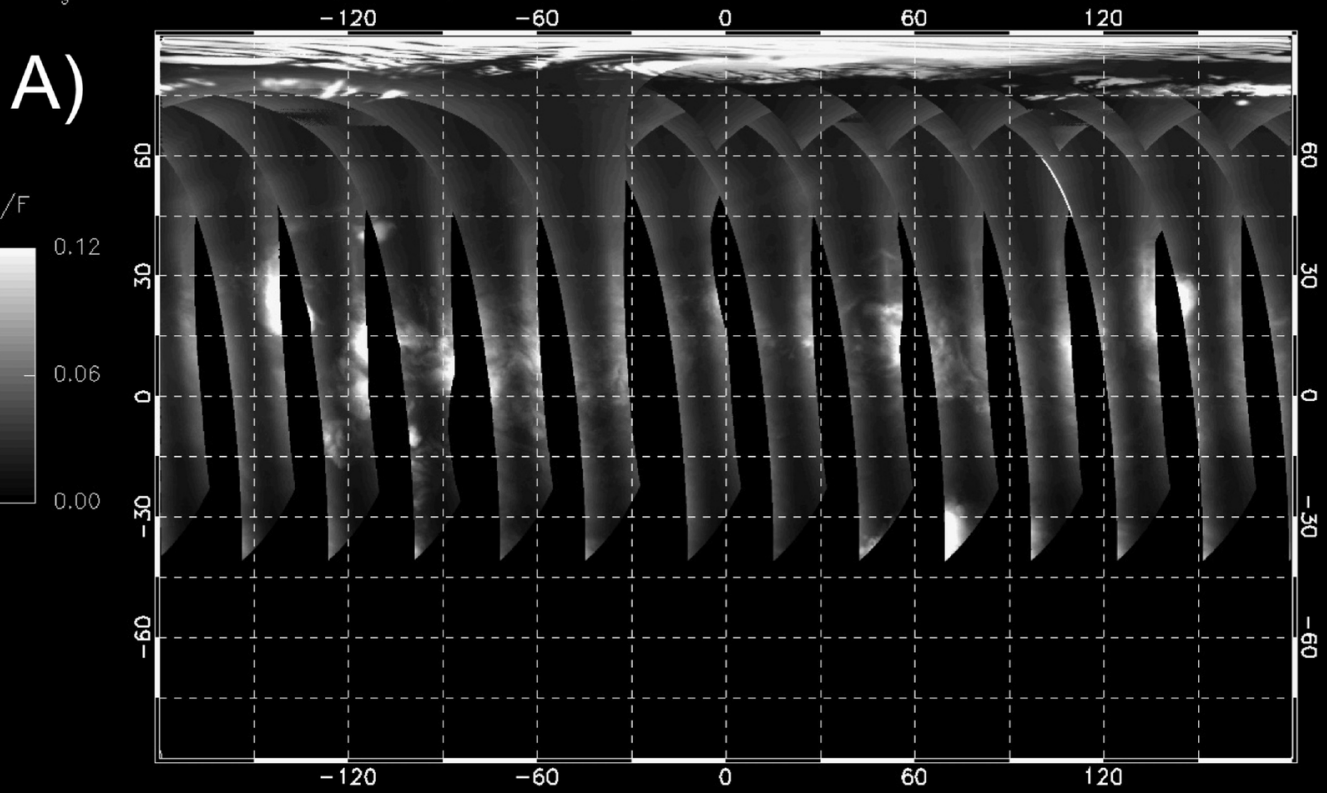

$L_{S}=298.1, M Y=29(6-J u l y-2009)$

East Longitude
$-120$
$-60$
0
60
120

B)

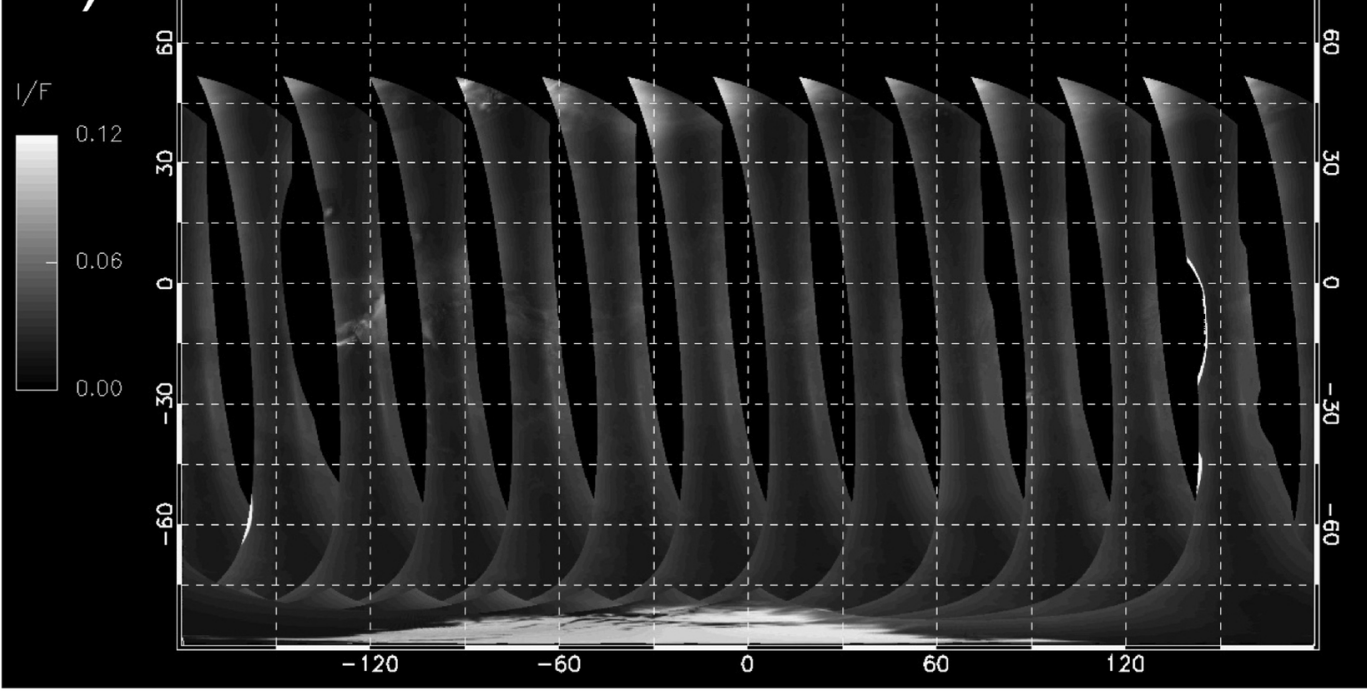




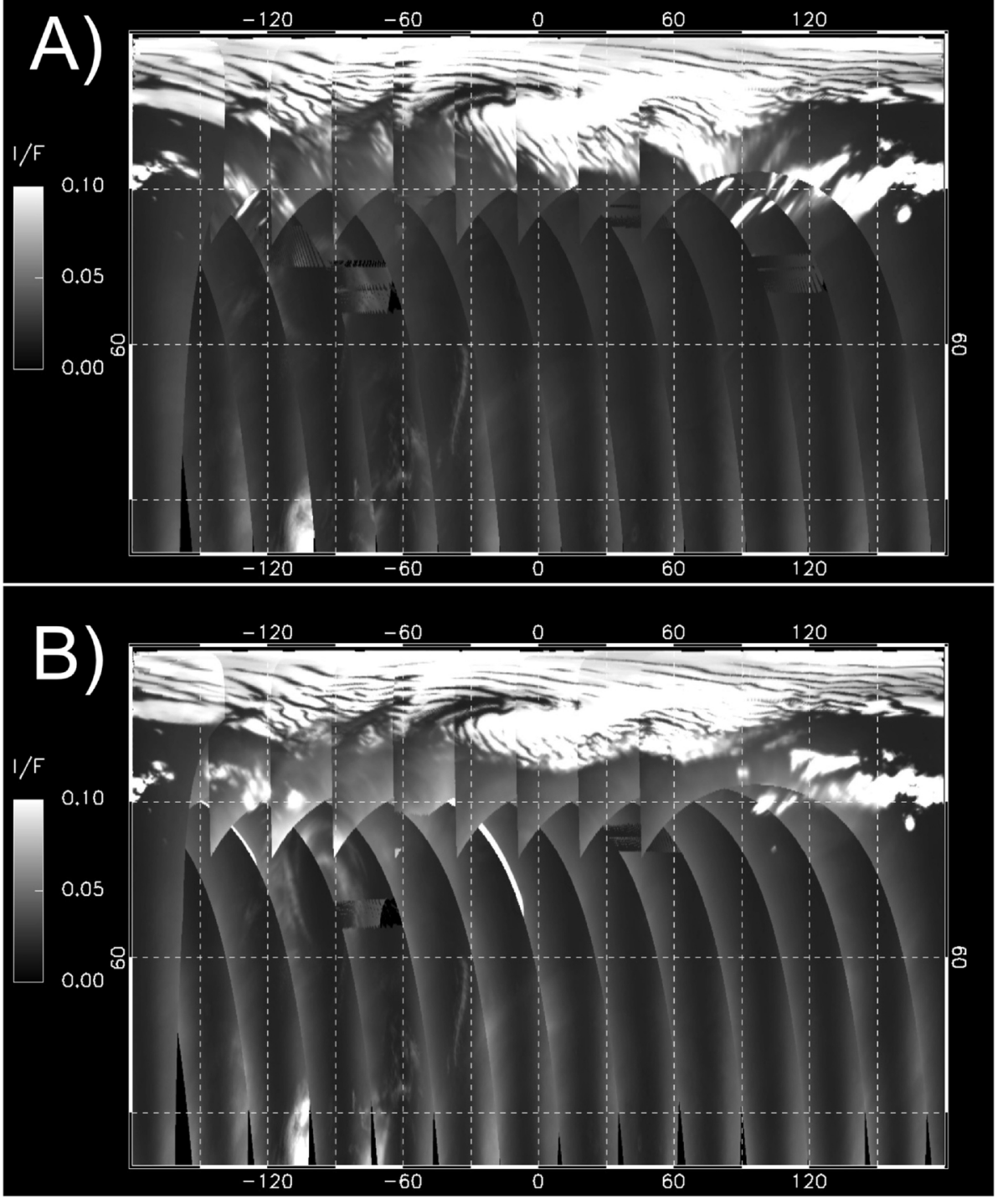

Figure 2 


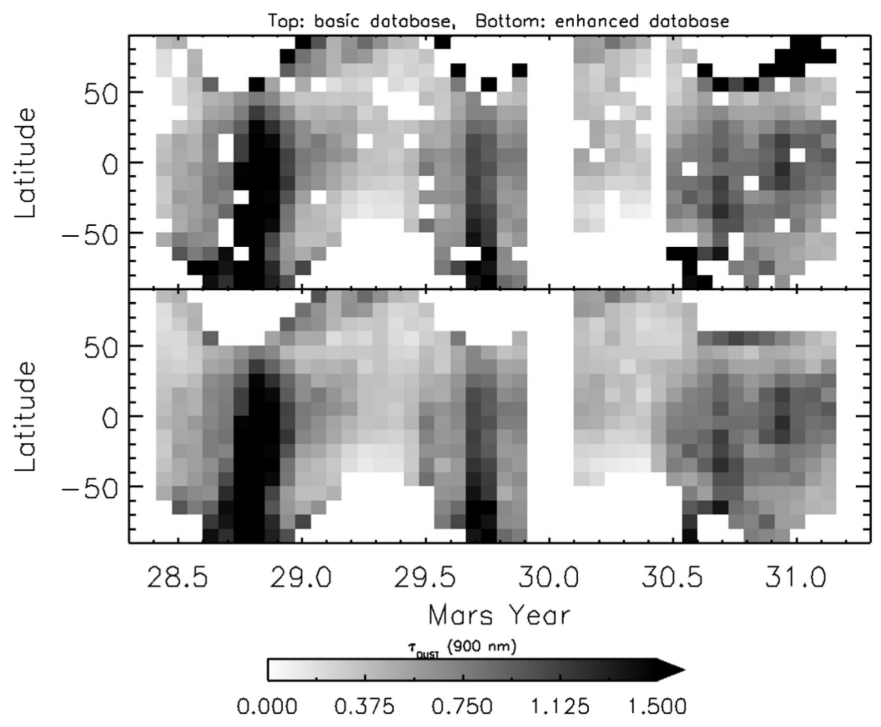

Figure 3 


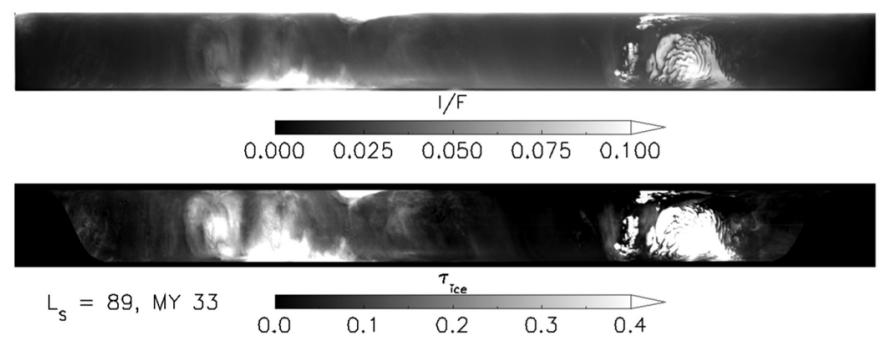

Figure 4 


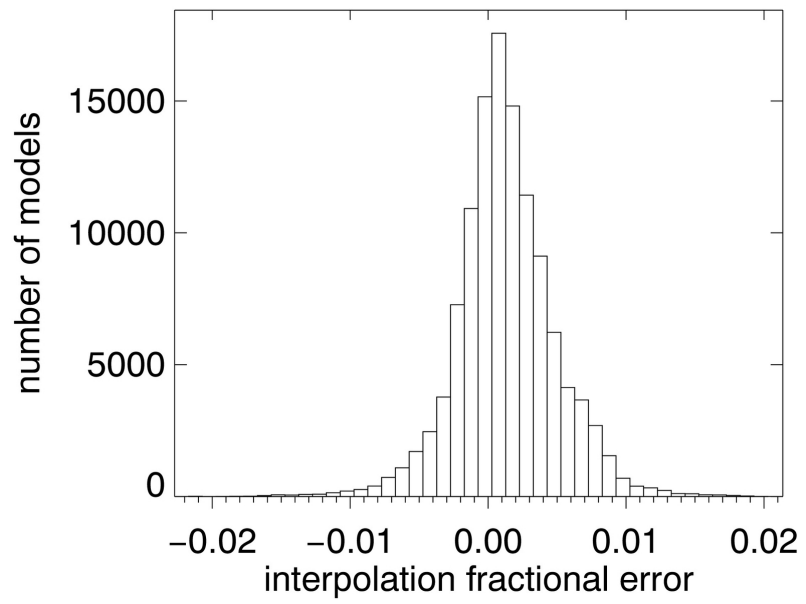

Figure 5 


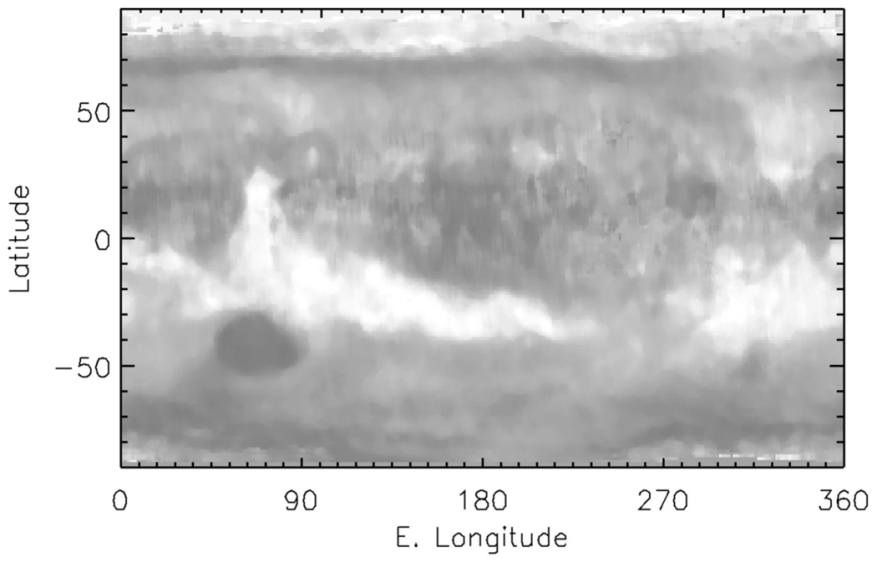

Hopke w

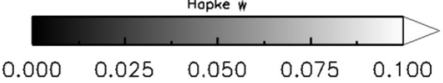

Figure 6 


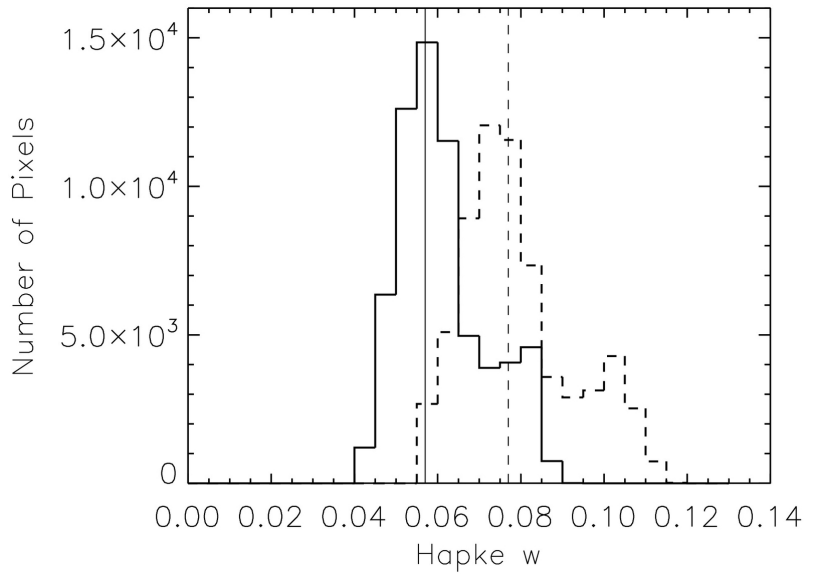

Figure 7 


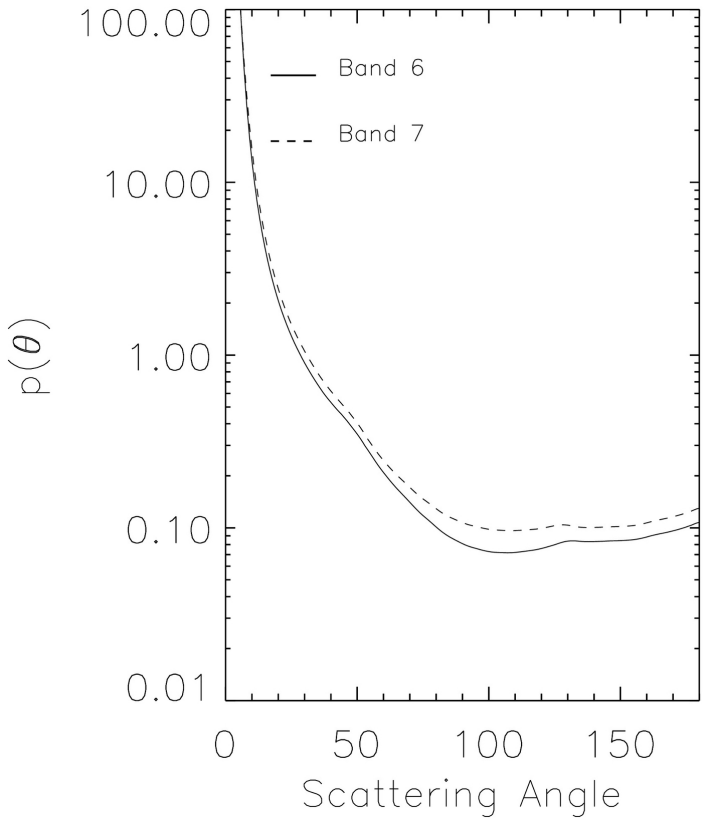

Figure 8 


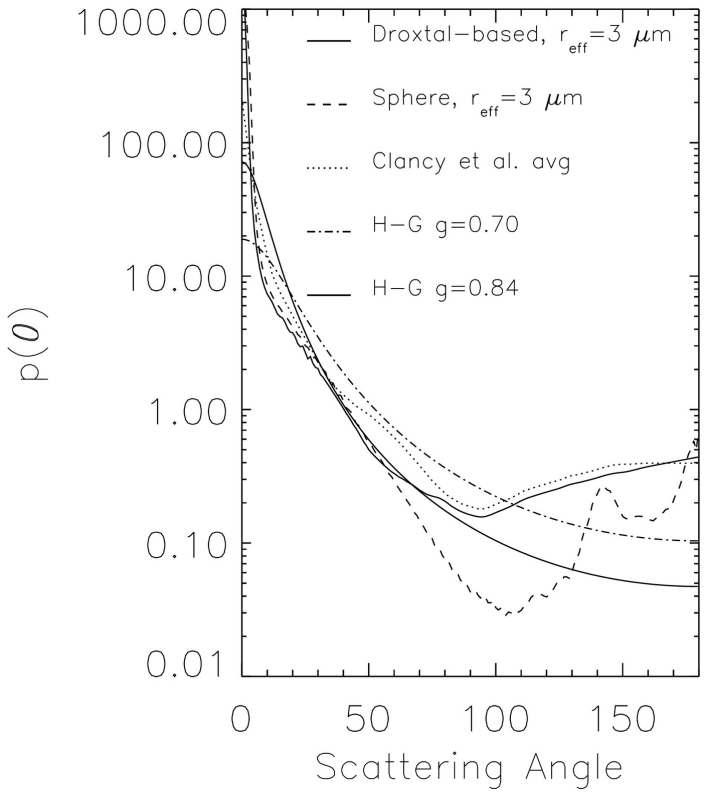

Figure 9 


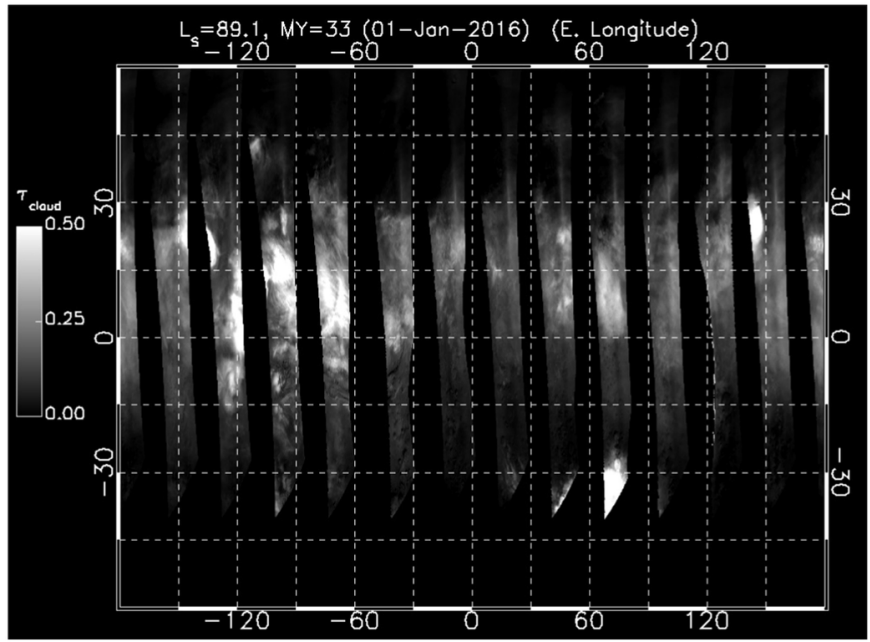

Figure 10 


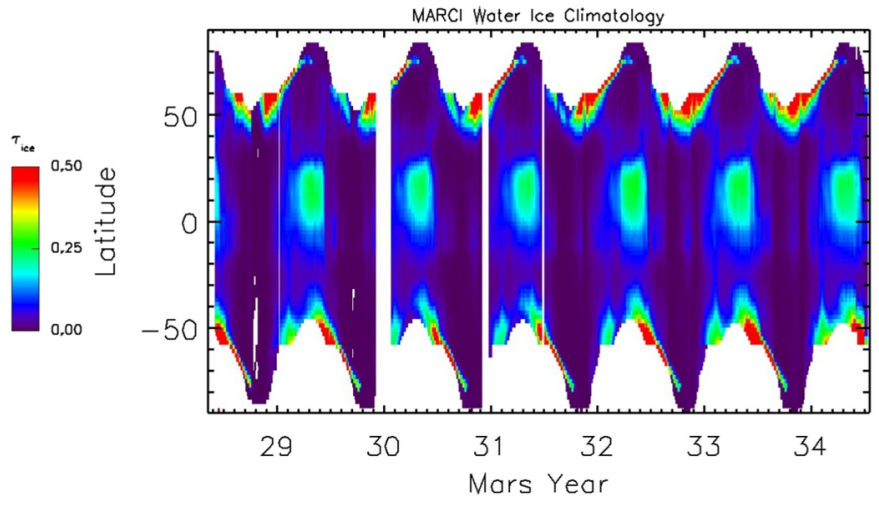

Figure 11 


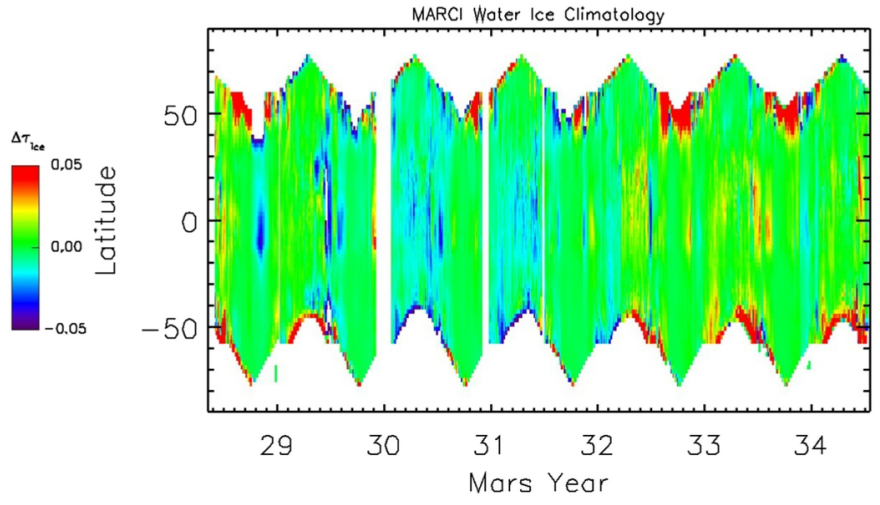

Figure 12 


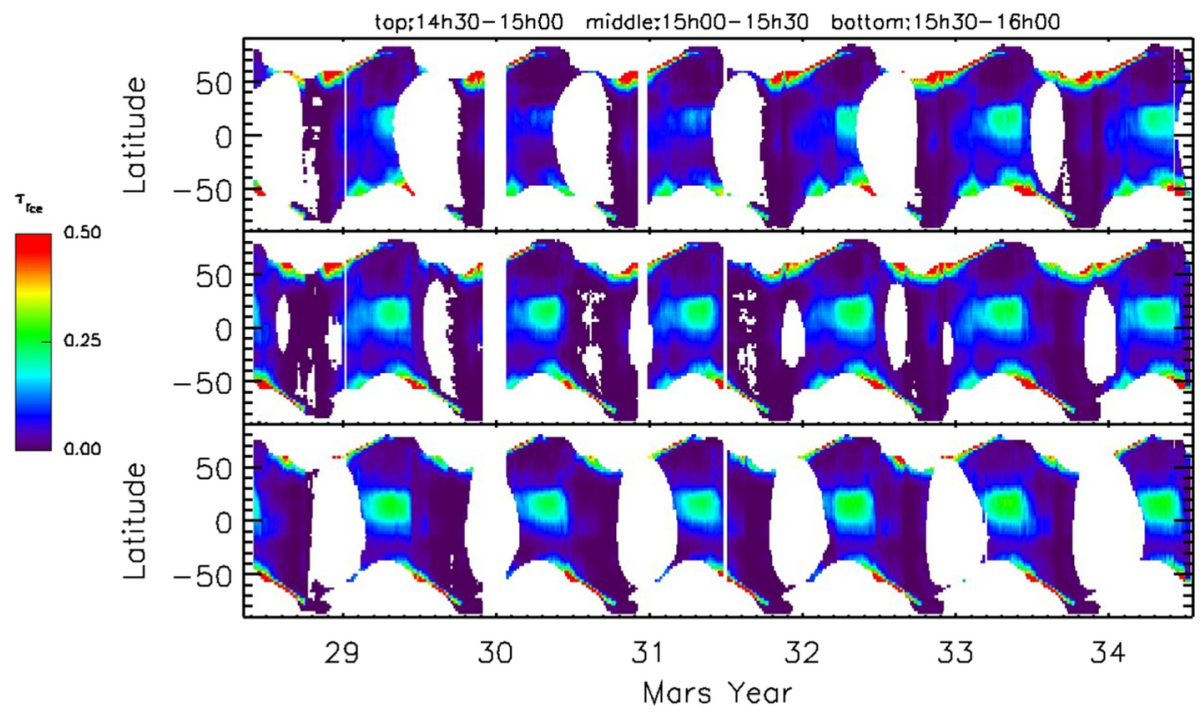

Figure 13 

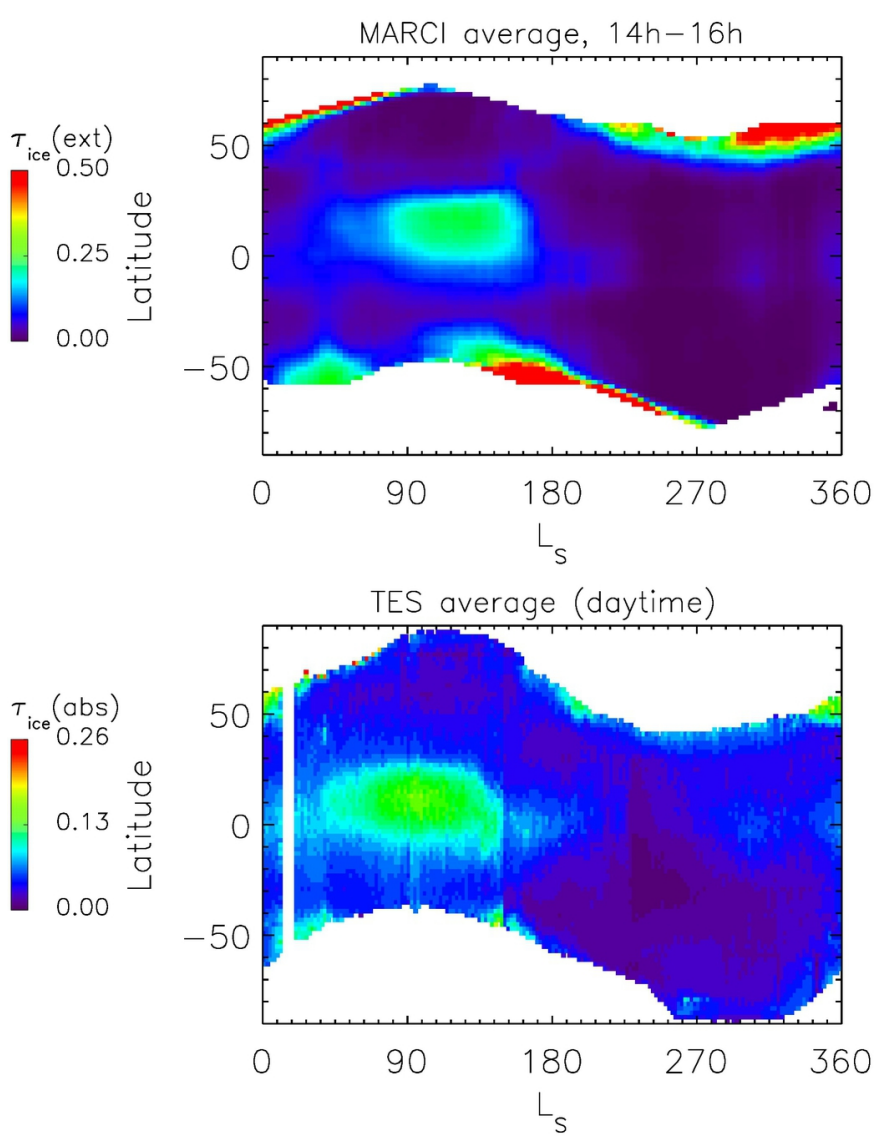

Figure 14 


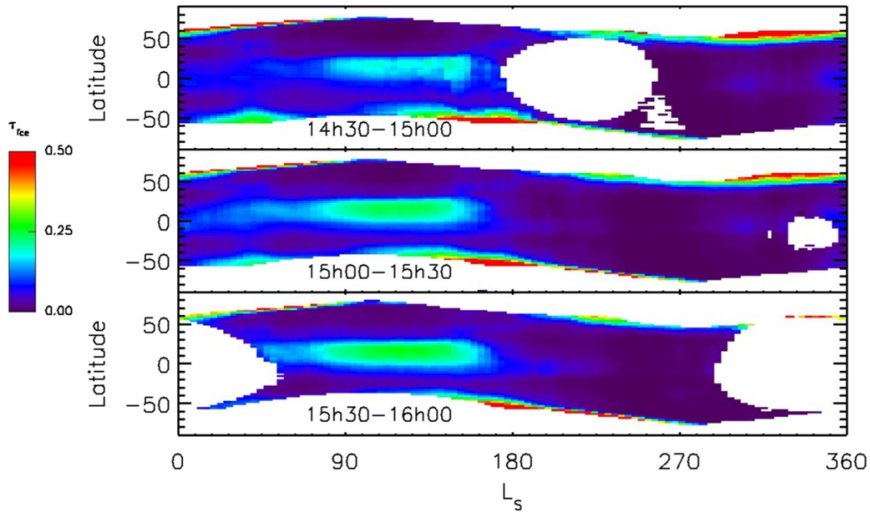

Figure 15 


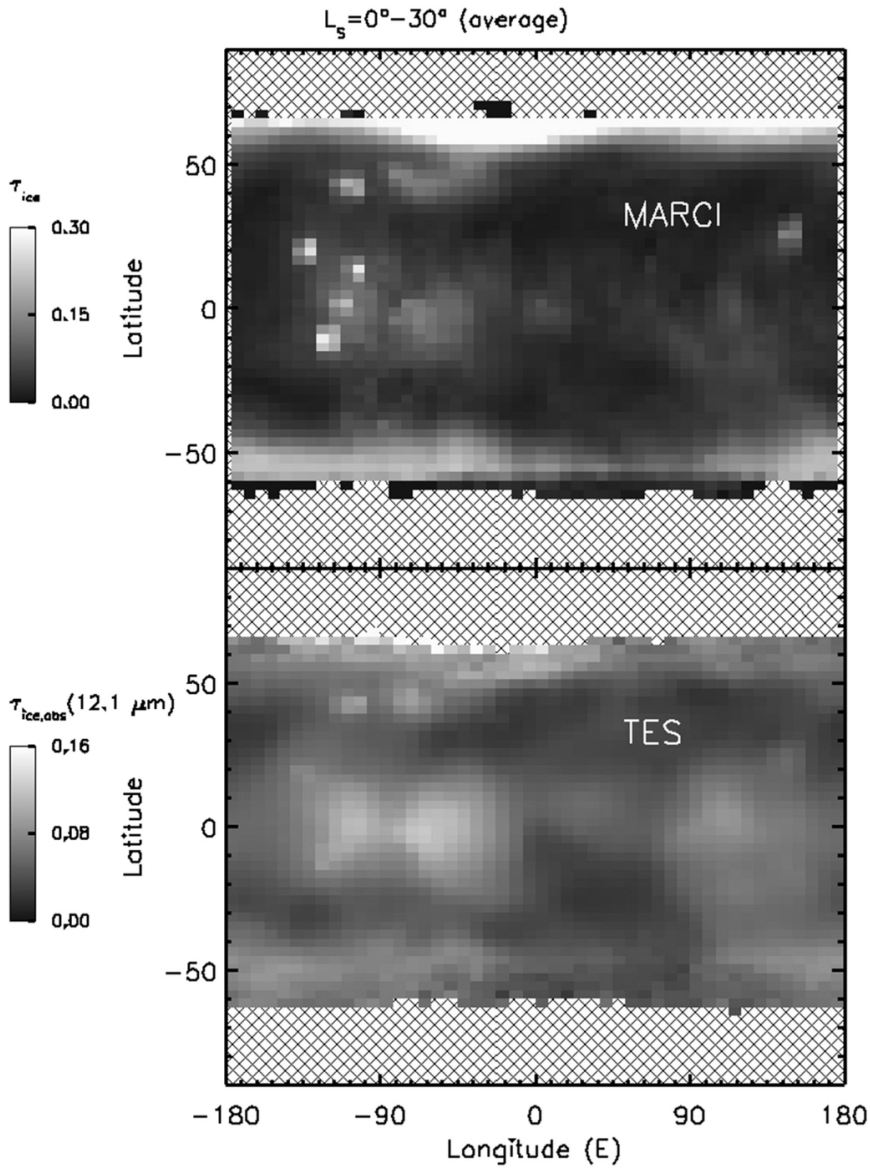

Figure 16 


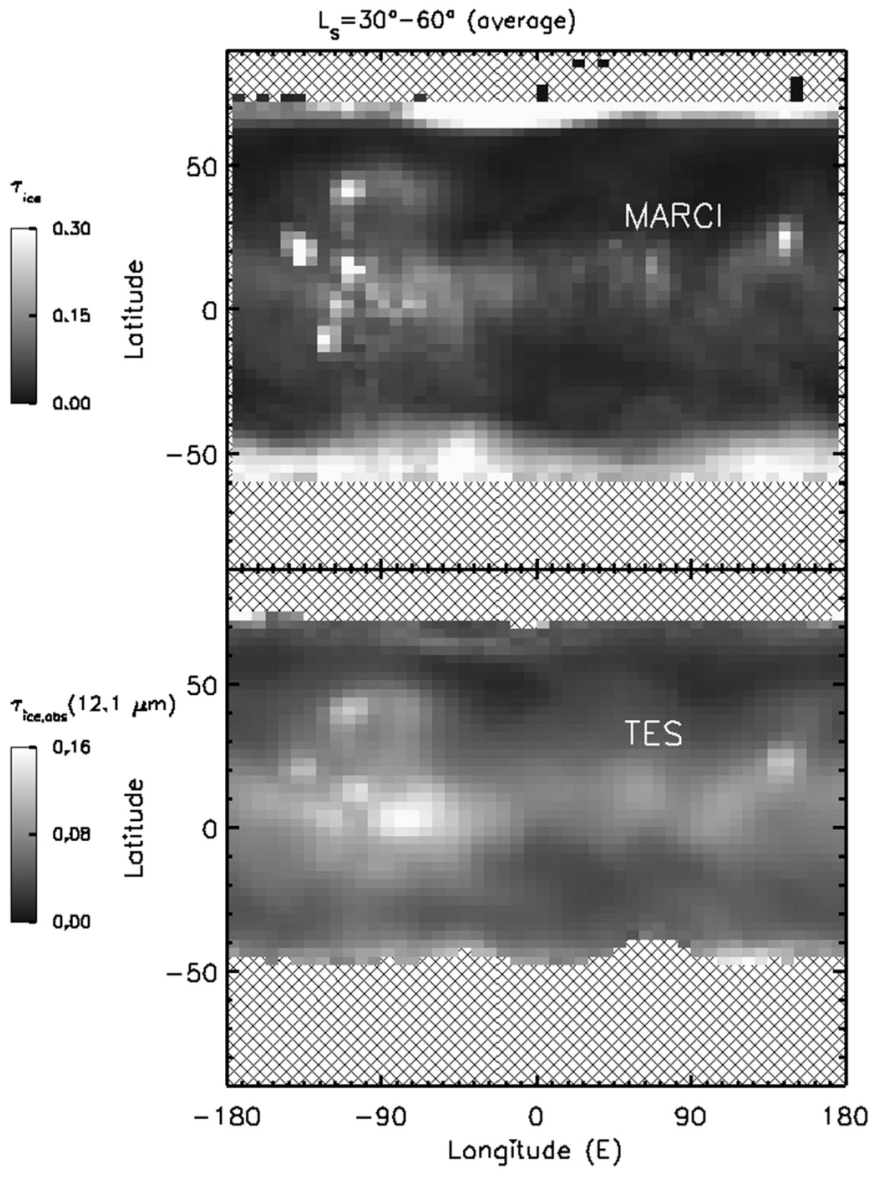

Figure 17 
$T_{\text {ice }}$

0.40

$-0.20$

0.00

$\tau_{\text {ice,abs }}(12,1 \mathrm{\mu m})$

$\left[\begin{array}{ll}0,21 & \\ 0,11 & \\ 0 & \end{array}\right.$

0,00

$$
\mathrm{L}_{\mathrm{s}}=60^{\circ}-90^{\circ} \text { (overoge) }
$$

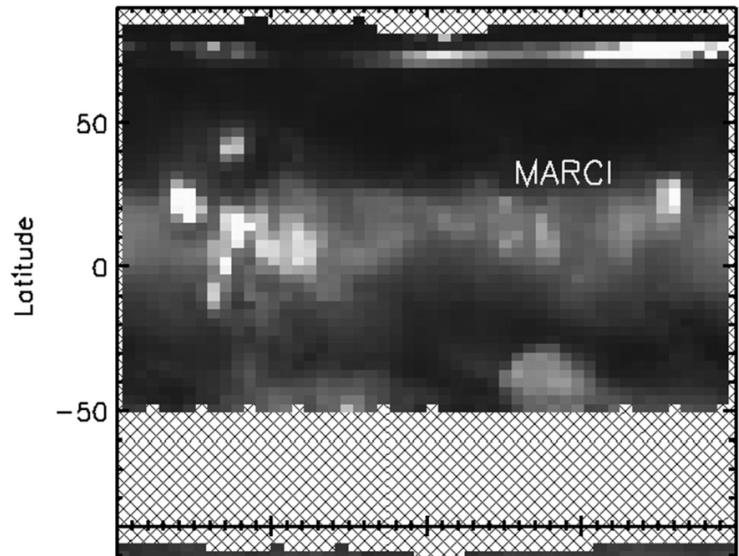

\section{TES}

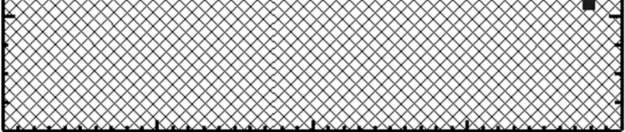

$-180$

$-90$

0

90

Longitude

(E)

Figure 18 
$T_{i c}$

0.50

0.00

$\tau_{\text {ice,abs }}(12,1 \mathrm{\mu m})$

$-50$

50

]

0,00

$-0.25$

므믐

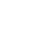

$$
\mathrm{L}_{\mathrm{s}}=90^{\circ}-120^{\circ} \text { (overoge) }
$$

50

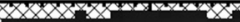
$\operatorname{coc}$
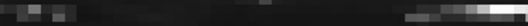

(1)

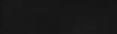

$+$

MAPC
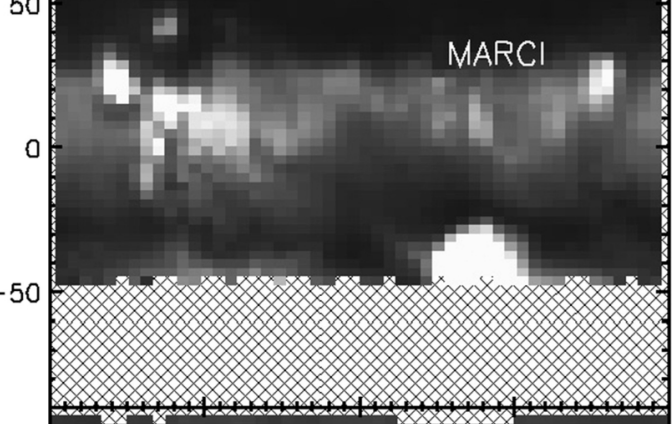

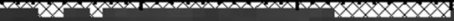

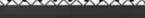

$-50$

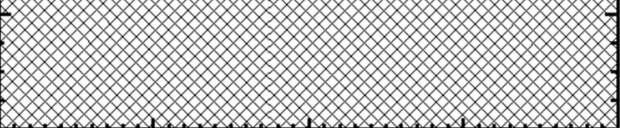

$-180$

$-90$

0

90

Longitude

TES

Figure 19 


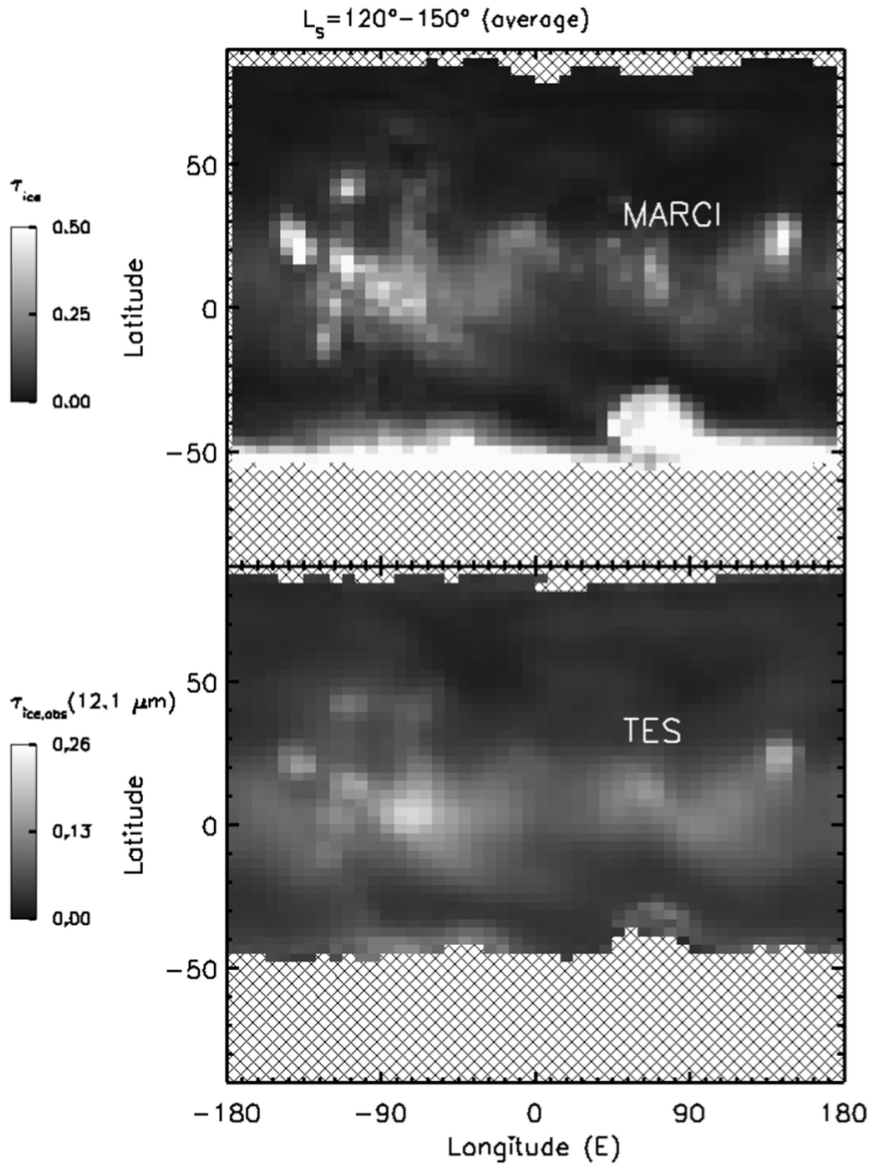

Figure 20 


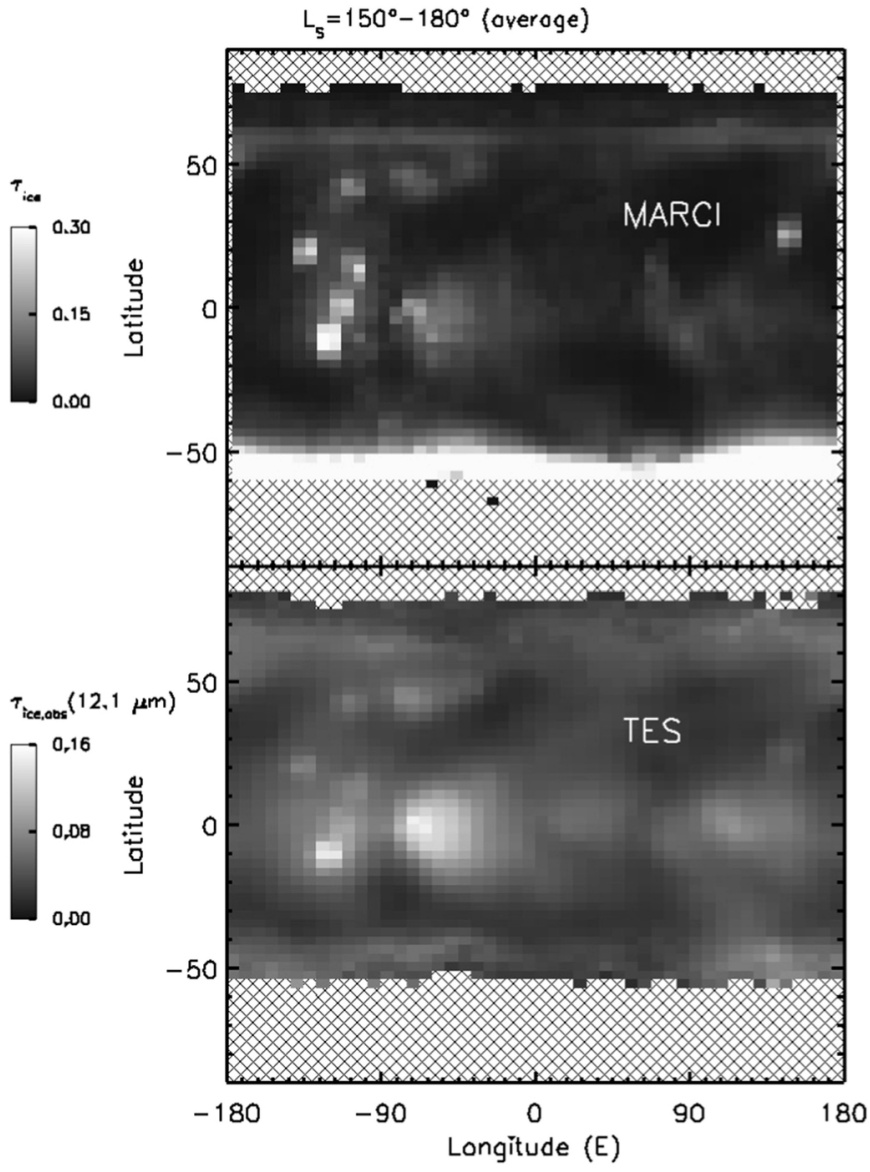

Figure 21 


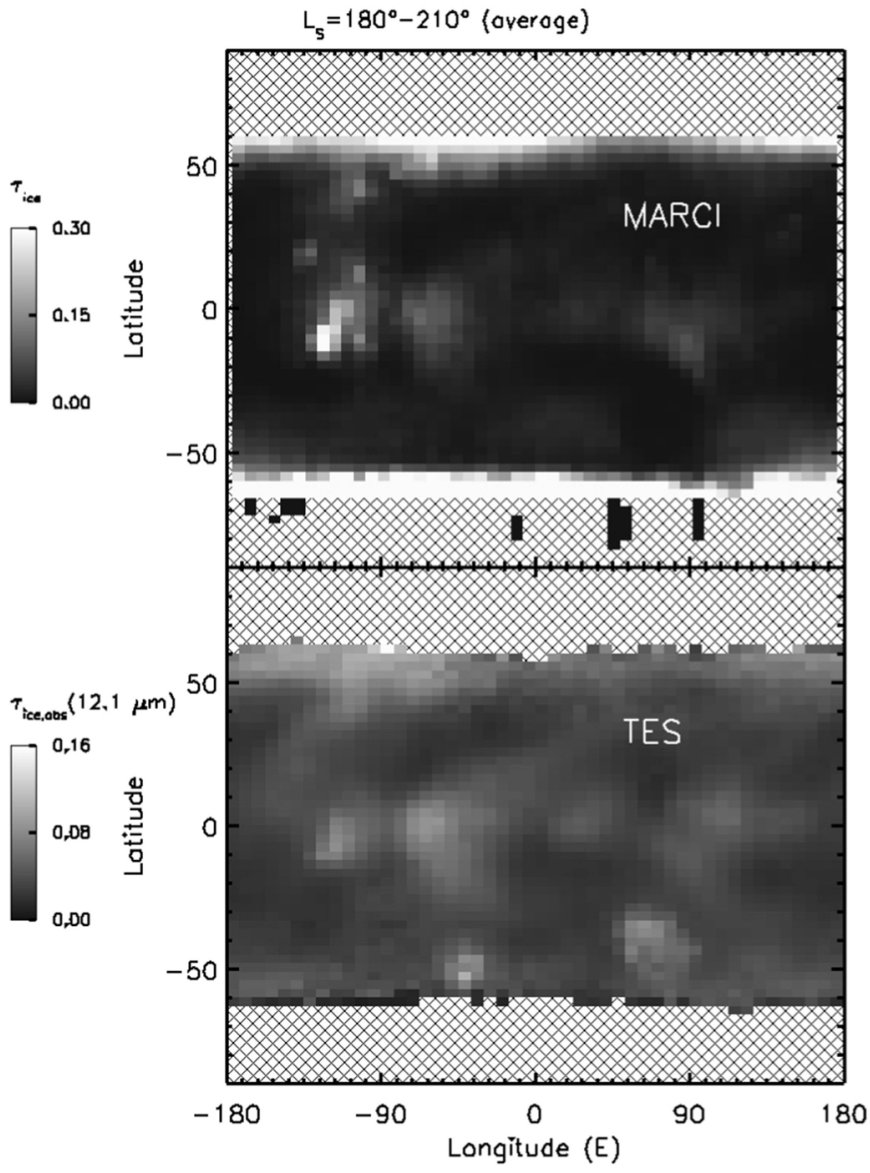

Figure 22 


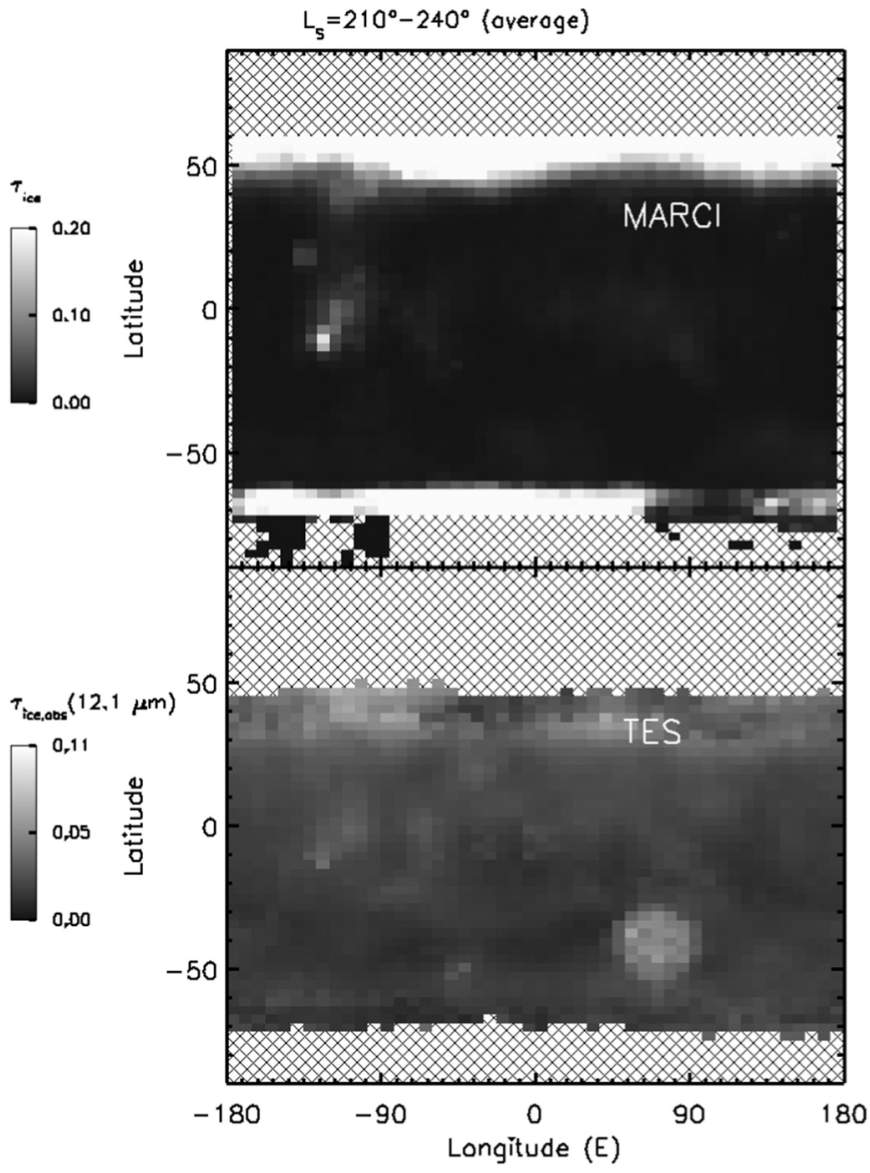

Figure 23 


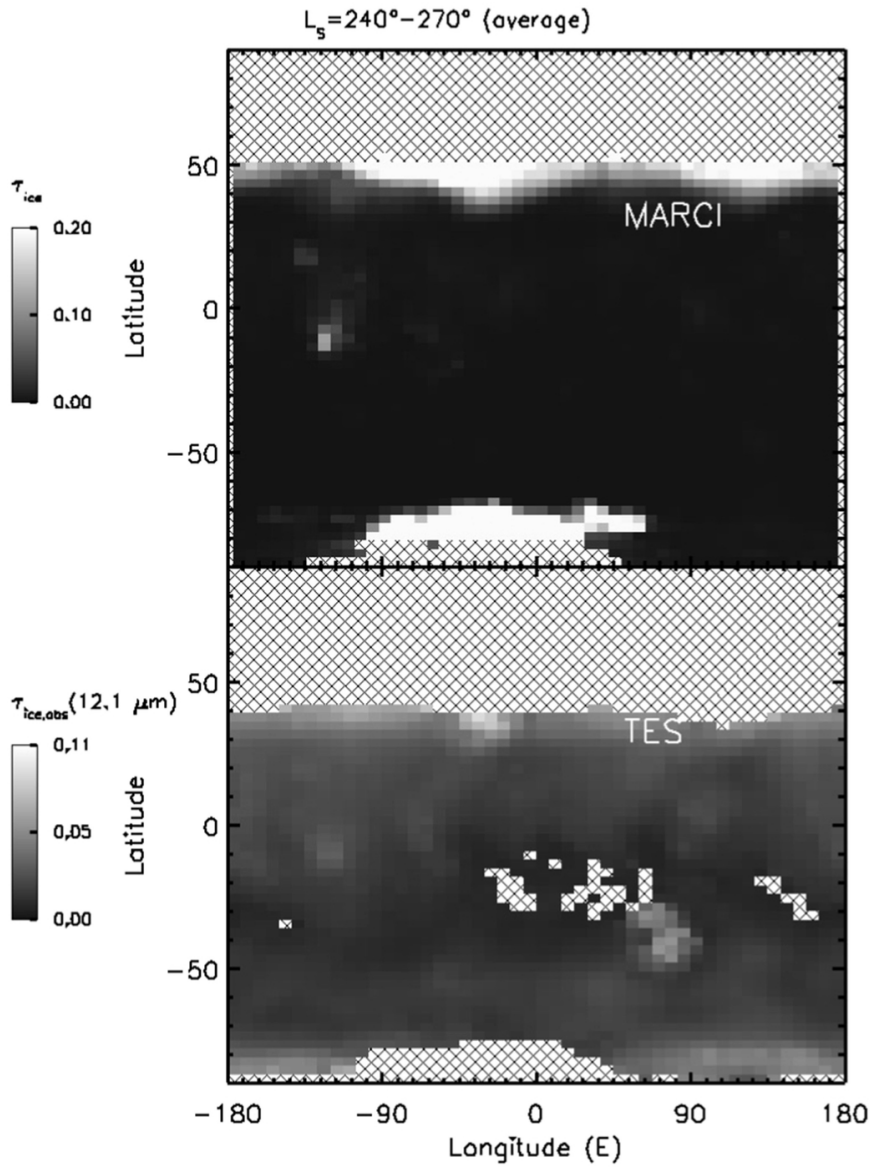

Figure 24 


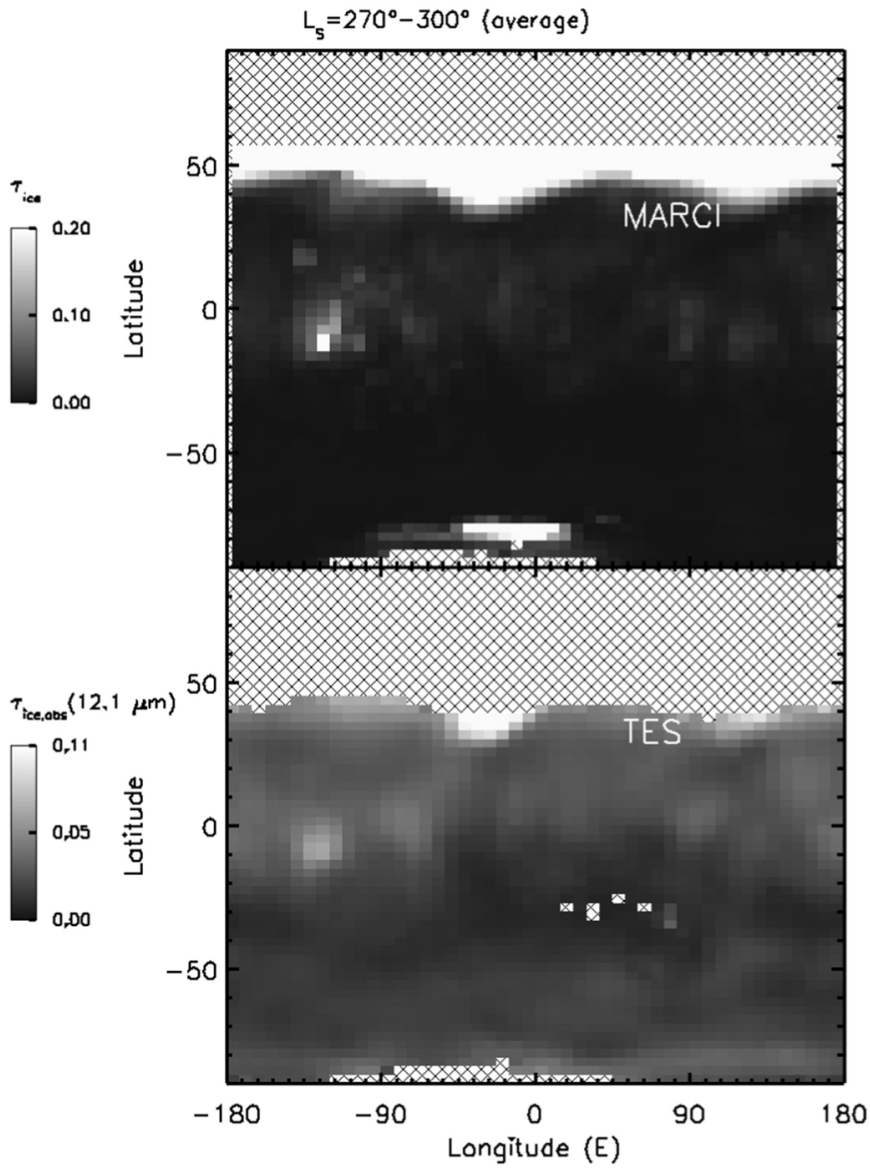

Figure 25 


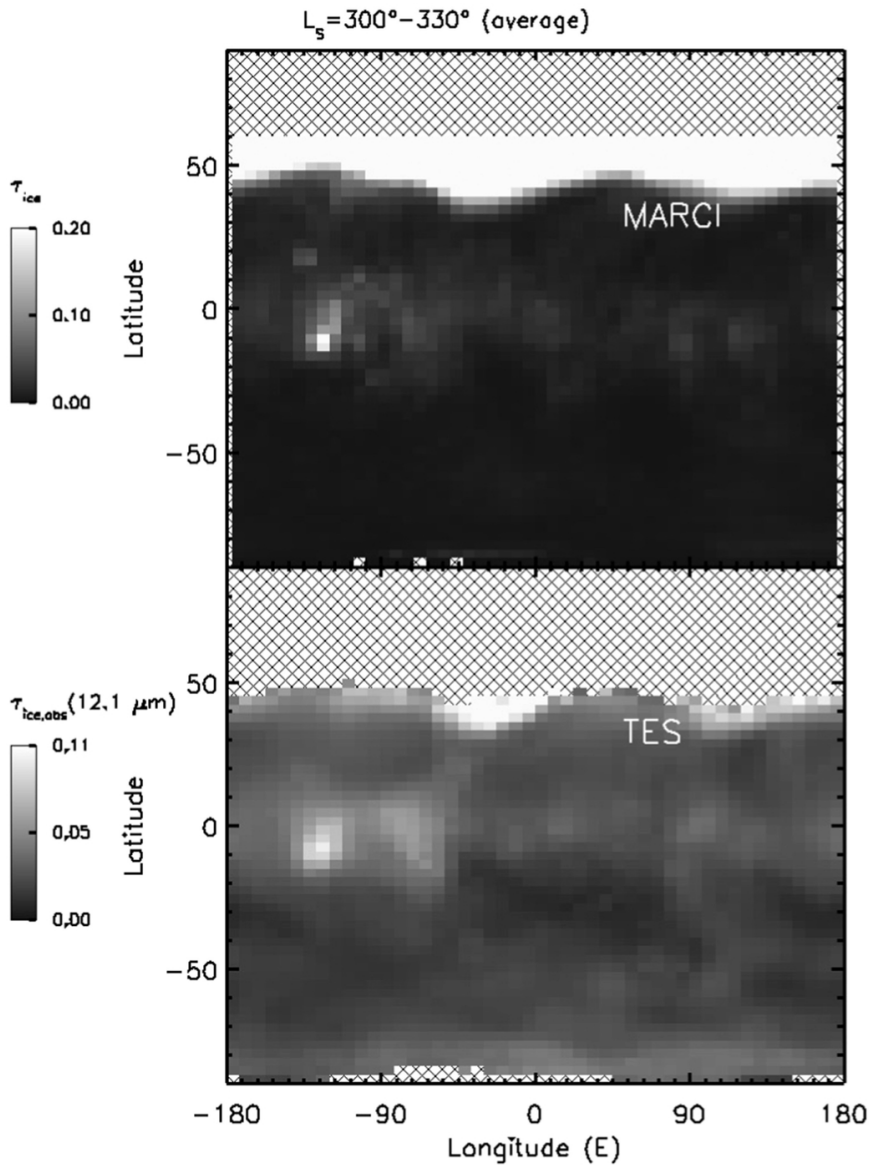

Figure 26 


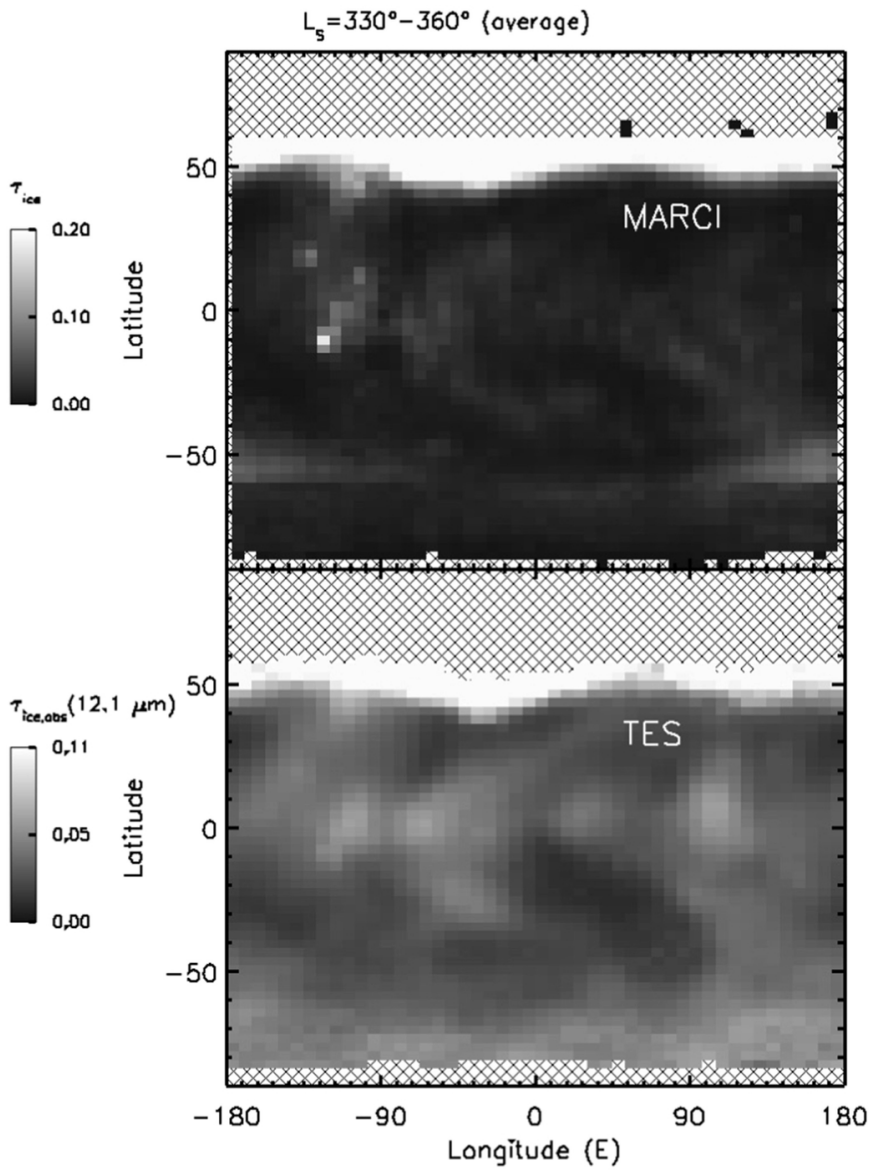

Figure 27 


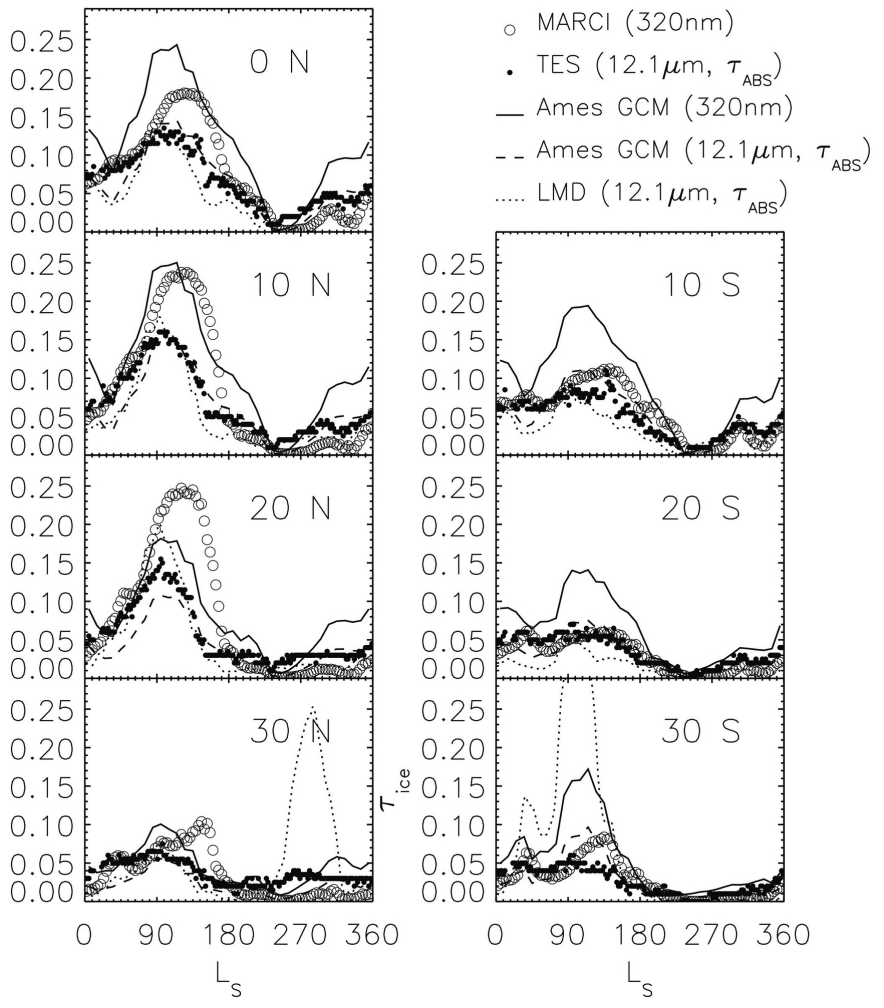

Figure 28 


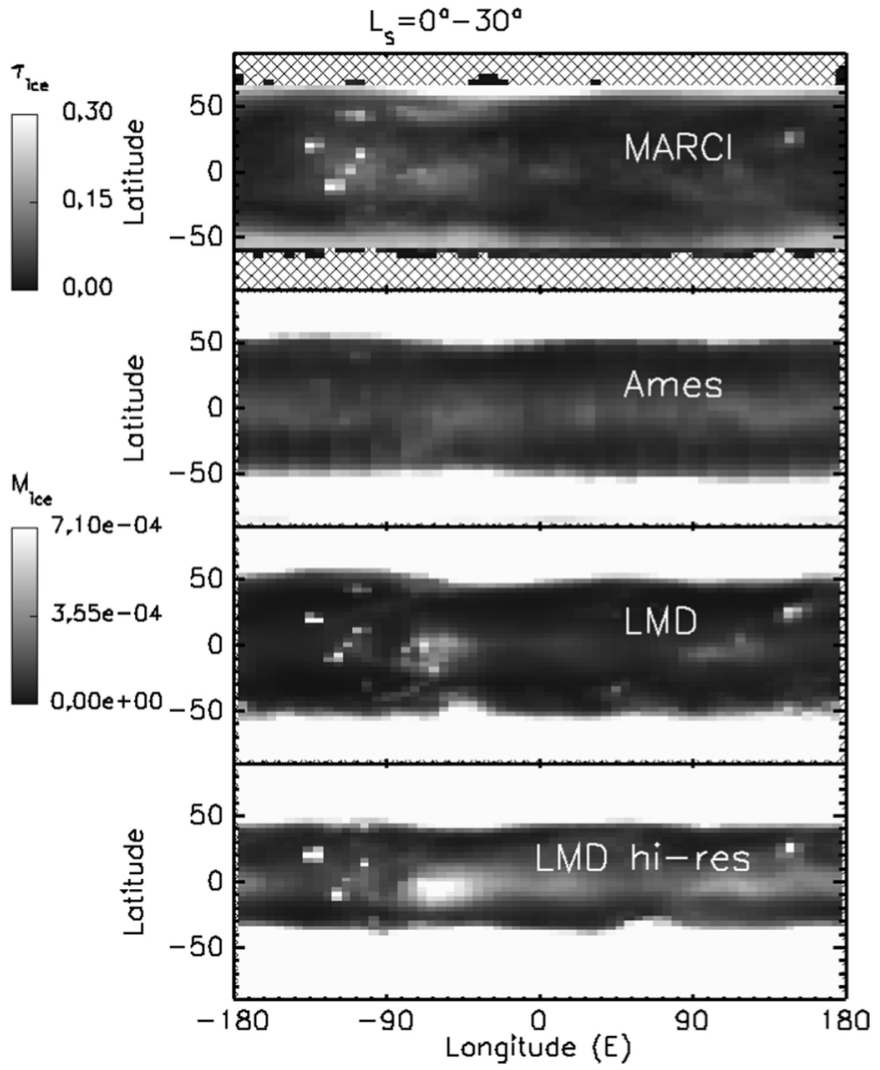

Figure 29 


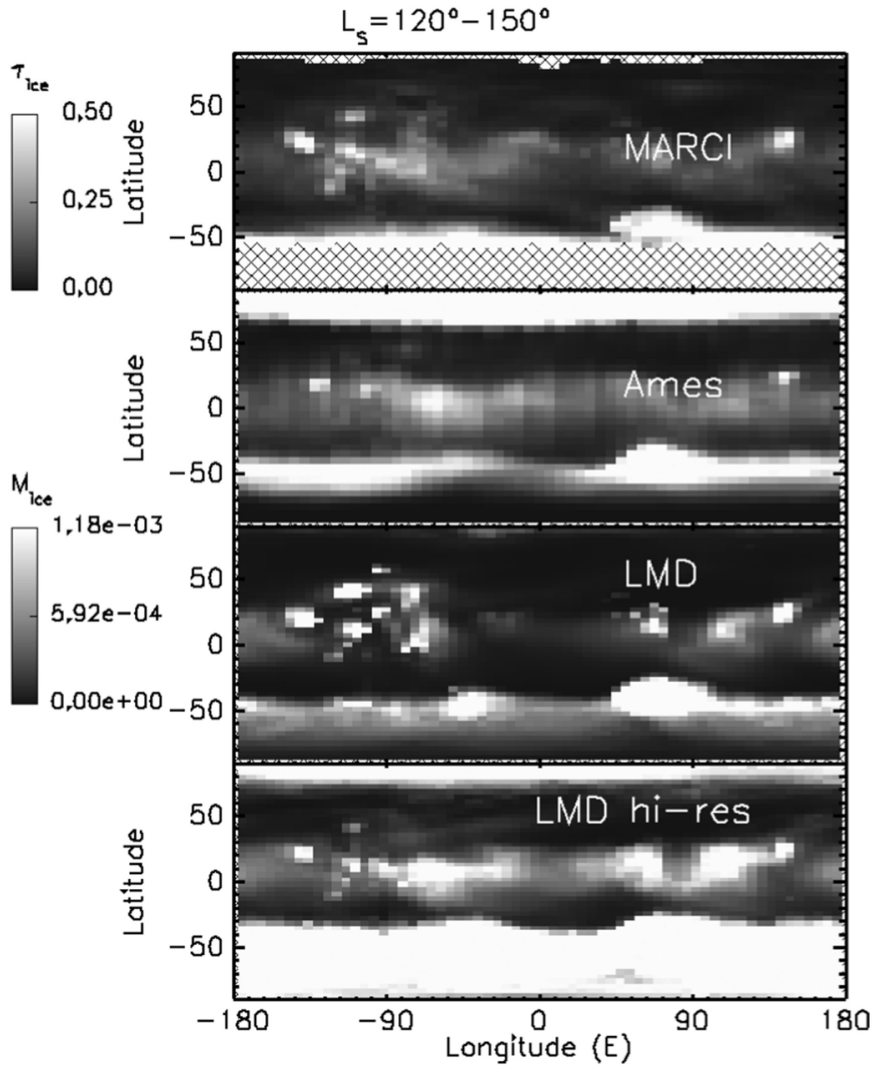

Figure 30 


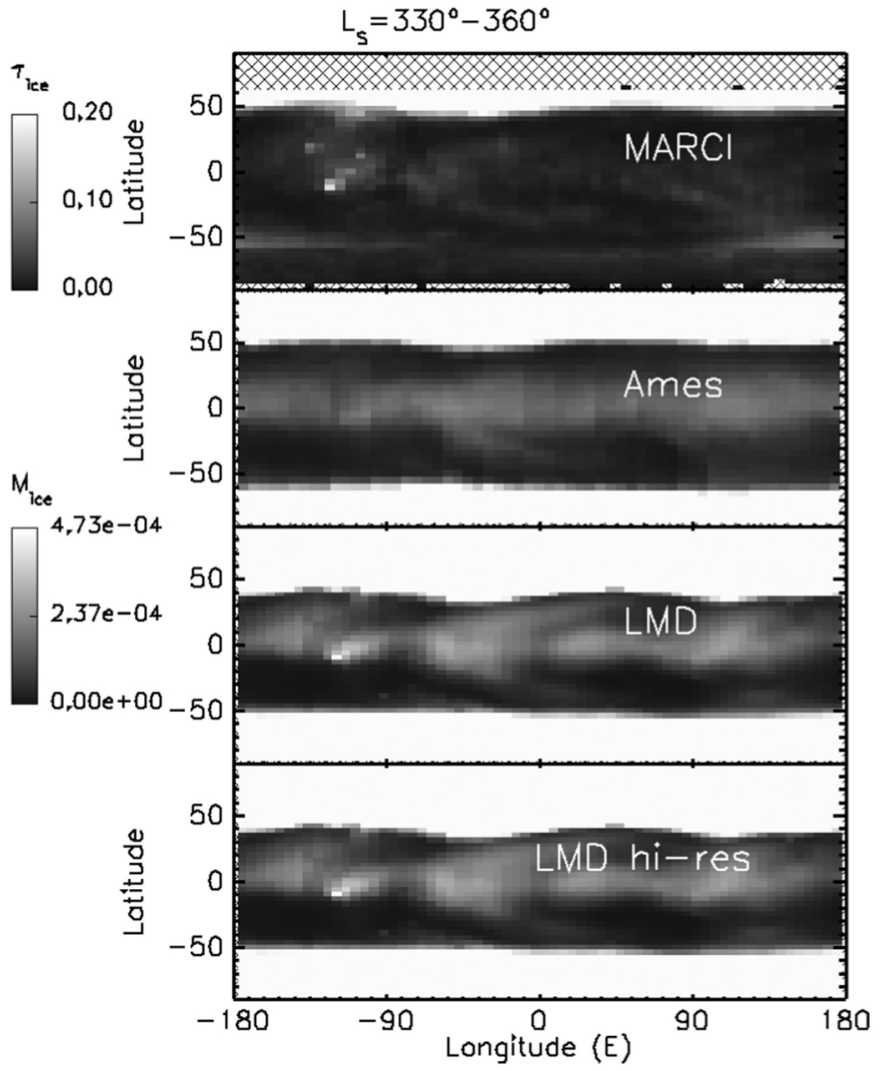

Figure 31 


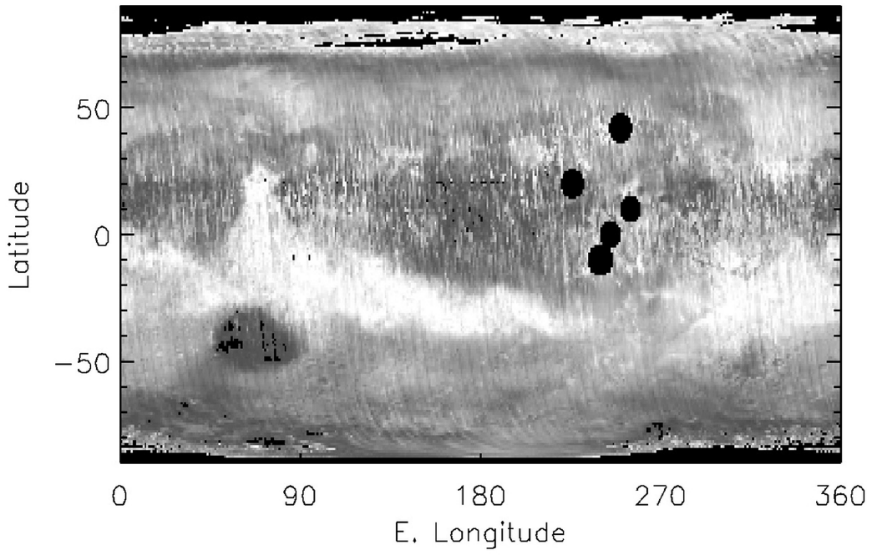

Hopke $\psi$

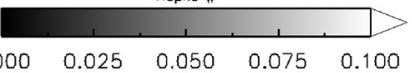

Figure 32 


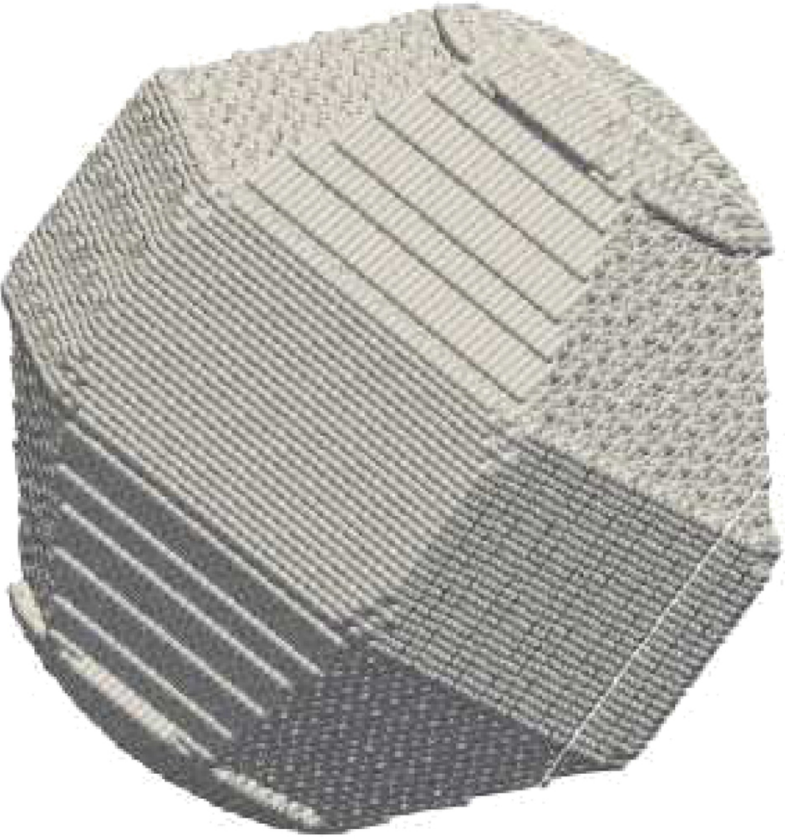

Figure 33 


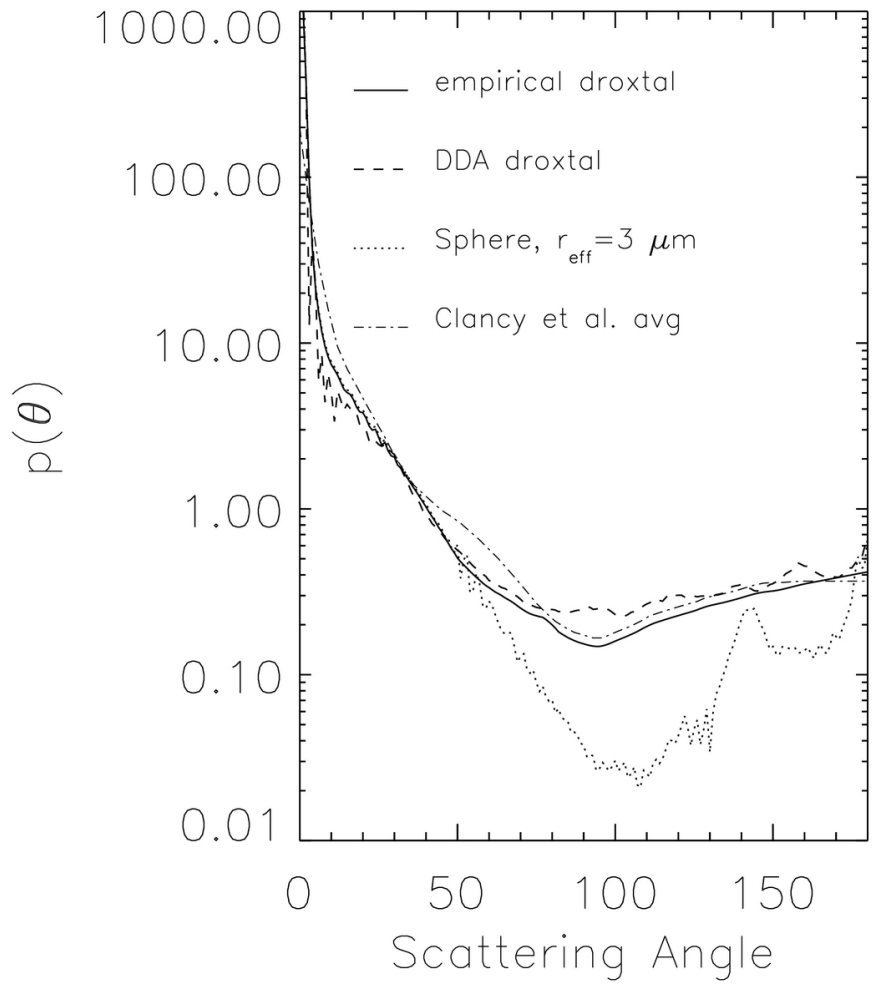

Figure 34 